\title{
Reproducibility of Thallium-201 Myocardial Perfusion Study in Rat Model with MicroSPECT
}

\author{
By \\ Munira Fardous Nahin
}

A Thesis Submitted to the Faculty of Graduate and Postdoctoral Affairs in Partial Fulfilment of the Requirements for the Degree of Master of Science

Ottawa-Carleton Institute for Physics

Department of Physics, Carleton University

Ottawa, Ontario, Canada

Copyright (C) Munira Fardous Nahin 2011 
Library and Archives

Canada

Published Heritage Branch

395 Wellington Street

Ottawa ON K1A ON4

Canada
Bibliothèque et

Archives Canada

Direction du

Patrimoine de l'édition

395 , rue Wellington

Ottawa ON K1A ON4

Canada
Your file Votre référence

ISBN: 978-0-494-81706-3

Our file Notre référence

ISBN: 978-0-494-81706-3
NOTICE:

The author has granted a nonexclusive license allowing Library and Archives Canada to reproduce, publish, archive, preserve, conserve, communicate to the public by telecommunication or on the Internet, loan, distribute and sell theses worldwide, for commercial or noncommercial purposes, in microform, paper, electronic and/or any other formats.

The author retains copyright ownership and moral rights in this thesis. Neither the thesis nor substantial extracts from it may be printed or otherwise reproduced without the author's permission.

\section{AVIS:}

L'auteur a accordé une licence non exclusive permettant à la Bibliothèque et Archives Canada de reproduire, publier, archiver, sauvegarder, conserver, transmettre au public par télécommunication ou par l'Internet, prêter, distribuer et vendre des thèses partout dans le monde, à des fins commerciales ou autres, sur support microforme, papier, électronique et/ou autres formats.

L'auteur conserve la propriété du droit d'auteur et des droits moraux qui protège cette thèse. $\mathrm{Ni}$ la thèse ni des extraits substantiels de celle-ci ne doivent être imprimés ou autrement reproduits sans son autorisation.
In compliance with the Canadian Privacy Act some supporting forms may have been removed from this thesis.

While these forms may be included in the document page count, their removal does not represent any loss of content from the thesis.
Conformément à la loi canadienne sur la protection de la vie privée, quelques formulaires secondaires ont été enlevés de cette thèse.

Bien que ces formulaires aient inclus dans la pagination, il n'y aura aucun contenu manquant.

\section{Canadä}




\section{Abstract \\ Gated myocardial perfusion Single Photon Emission Computed Tomography}

(SPECT) is a non-invasive diagnostic medical imaging test and one of the most commonly used medical procedures for assessing myocardial perfusion and left ventricular functions in the same study. Serial imaging with SPECT is used to study heart diseases in small animals and thereby can play a role in developing new therapies and new radiotracers and also can follow disease progression inside the subject. In vivo measurement using microSPECT allows the evaluation of cardiac factors like end diastolic volume (EDV), end systolic volume (ESV), ejection fraction (EF) and perfusion homogeneity $(\mathrm{PH})$. Serial imaging reduces the unnecessary sacrifice of small animals and improves the power of small-animal studies. The goal of our study is to determine the intra- and inter-subject reproducibility of left ventricular functional measurements with T1-201 thallium-chloride for cardiac microSPECT imaging in a rat model. Eight normal Sprague-Dawley rats underwent electrocardiogram-gated SPECT scanning weekly for 5 weeks and eight rats with a surgically induced myocardial infarction underwent imaging for 2 weeks at 2-3 day intervals. The rats were injected with $0.5 \mathrm{mCi}$ of Tl-201 and scanned with a mciroSPECT scanner. The microSPECT was a 4-headed scanner with 9 pinholes/head ( 36 pinholes in total). The scan was started just after the injection and continued for 30 minutes. We used 4DM-SPECT, a clinical software package, to analyse the data. We found the values of EDV, ESV, EF and $\mathrm{PH}$ and the intra- and inter-subject variance in these values for both normal and MI rats for 10 minute, 20 minute and 30 minute scanning durations. There were no significant differences found between myocardial infarcted and normal rats with respect to the intra-subject variability, but there were significant differences in the inter-rat variability. This study provides a baseline reproducibility of Tl-201 for cardiac microSPECT small-animal imaging. 


\section{Acknowledgement}

I humbly desire to express my deepest and most sincere gratitude, and immense indebtedness to my respected supervisor, Dr. Glenn Wells, for his encouragement, helpful advice, suggestions and active co-operation in preparing this paper. He has taken great pains to guide me. Not only he encouraged and helped me at each stage of the study but also he provided appropriate ideas in designing the study and sharpening the focus of the study.

I am grateful to my ever-respected honourable teachers of the Department of Physics, Carleton University, especially to Dr. Dave Rogers, Dr. Paul Johns, Dr. Gerald Oakham and Dr. Tong $\mathrm{Xu}$, who extended their co-operation and help at various stages of this study.

Many researchers and organizations helped me by providing useful references and access to reports. I am grateful to them.

Finally, I feel highly indebted to Julia Lockwood and all of my colleagues at Cardiac research of University of Ottawa Heart Institute, Ottawa, Canada.

This work is dedicated to my parents. 


\section{Table of Contents}

Title

Page

Abstract

Acknowledgement

Table of Contents

List of Tables viii

List of Figures $\quad$ ix

Glossary of Acronyms xiv

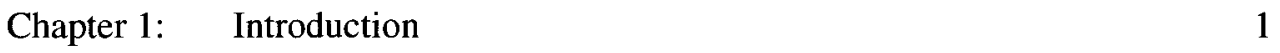

1.1 Cardiac Disease and SPECT 1

1.1.1 Around the World 1

1.1.2 Around Canada 3

1.1.3 Role of SPECT Imaging 4

1.1.4 How it Works: Tracer Technology 5

1.1.5 Parameters Measured with Cardiac SPECT Studies 8

$\begin{array}{ll}\text { 1.2 Cardiac SPECT Imaging Physics } & 10\end{array}$

$\begin{array}{lll}\text { 1.2.1 Clinical SPECT } & 10\end{array}$ 
2.3.4.1 Comparison between $\mathrm{Tc} 99^{\mathrm{m}}$-tetrofosmin and Tl-201 Study 80 
Chapter 3: Conclusion

References

Appendices 


\section{List of Tables}

Title

Page

Table $1.1 \quad$ : Common SPECT radionuclides used for imaging

Table 1.2 : Commonly used radiopharmaceuticals in cardiac SPECT

Table 1.3 : Performance characteristics of clinical and preclinical SPECT

Table 2.1 : Physiological data (Heart Rate (bpm) and Respiratory Rate (per minute)) of normal and MI rats

Table $2.2 \quad$ : Average standard deviation of observed measures

Table 2.3 : Variability as a percentage of EDV and ESV

Table 2.4 : Comparison of our Tl-201 study with a Tc99m-tetrofosmin (Tc99m) study 


\section{List of Figures}

Title

Page

Figure $1.1 \quad$ : Basic components of a typical gamma camera

Figure $1.2 \quad$ : Four different types of events that are detected by a

gamma camera

Figure 1.3 : Diagram of several SPECT systems with different number

of gamma cameras

Figure 1.4 : Schematic representation of iterative reconstruction method

Figure 1.5 : Different reconstructed images

Figure 1.6 : Cardiac plane definition for tomographic display

Figure 1.7 : Polar map representation with the corresponding 3D LV image. 24

Figure $1.8 \quad$ : 17 segment model of left ventricle (LV)

Figure 1.9 : Schematic diagram of a single pinhole collimator, imaging an object, showing how it produces a magnified image of the object

Figure 2.1 : Sprague-Dawley Rats - which we were working with

Figure 2.2 : MicroSPECT scanner at University of Ottawa Heart Institute

Figure 2.3 : Cardiac reorientation of left ventricular long axis slices 
Figure 2.4 : Example of an image of a normal rat displayed in the 4DM-SPECT

software package

Figure 2.5 : Weights of the 8 normal rats during the study

Figure 2.6 : EDV and ESV for 8 normal rats (uncorrected) and 5 different scans using a 10 minute scan duration

Figure 2.7 : Weight-corrected EDV and ESV of 10 minute scan for the 8 normal rats

Figure $2.8 \quad$ : Ejection fraction and perfusion homogeneity of 8 normal rats for a 10 minute scan duration

Figure 2.9 : Polar map of normal database for 10 minute scan duration

Figure 2.10 : Reproducibility of uncorrected EDV and ESV for inter- and intra-subject of 8 normal rats for 20 minute scan

Figure 2.11 : Reproducibility of weight corrected EDV and ESV for 20 minute scan duration of 8 normal rats

Figure 2.12 : Inter- and intra-subject reproducibility of EF and $\mathrm{PH}$ for

a 20 minute scan duration of 8 normal rats

60

Figure 2.13 : Polar map of normal database for 20 minute study

Figure 2.14 : Inter- and intra-subject reproducibility of uncorrected EDV and ESV of 30 minute scan of 8 normal rats 
Figure 2.15 : Inter- and intra-subject reproducibility for EDV (corrected)

and ESV (corrected) for the 30 minute study of 8 normal rats

Figure 2.16 : Variability of EF and PH for 30 minute scan duration of

8 normal rats

64

Figure 2.17 : Polar map of normal database for 30 minute scan

Figure 2.18 : The variability of weight of MI rats during the experiment

Figure 2.19 : Inter- and intra-subject reproducibility of uncorrected EDV

and ESV for a 10 minute scans of MI rats

67

Figure 2.20 : Inter- and intra- subject reproducibility of weight corrected

EDV and ESV for a 10 minute study of $8 \mathrm{MI}$ rats

Figure 2.21 : Inter- and Intra-subject reproducibility of EF and PH for

10 minute scan of $8 \mathrm{MI}$ rats

69

Figure 2.22 : Z-scores of the measurement of tracer uptake for $8 \mathrm{MI}$ rats for the 10 minute scans

Figure 2.23 : The inter- and intra-subject reproducibility of uncorrected

EDV and ESV for 20 minute scans duration of MI rats

Figure 2.24 : The inter- and intra-subject reproducibility of corrected

EDV and ESV for 20 minute scans of MI rats 
Figure 2.25 : Reproducibility of EF and PH, across scans and across

rats, for a 20 minute study of MI rats

Figure $2.26:$ Z-scores of the measurement of tracer uptake of 8 MI rats

for 20 minute scans

Figure 2.27 : Inter- and intra-subject reproducibility of uncorrected

EDV and ESV for 30 minute scans for 8 MI rats

Figure 2.28 : Reproducibility of corrected EDV and ESV for intra- and inter-subject for 30 minute study of MI rats

Figure 2.29 : Reproducibility of intra- and inter-subject EF and PH for 30 minute study of MI rats

Figure 2.30 : Z-scores for the measurement of tracer uptake of $8 \mathrm{MI}$ rats for 30 minute study

Figure 2.31(a) : Comparison of time duration for the uncertainty in the end diastolic volume (EDV) measurement of normal rats

Figure 2.31(b) : Comparison for the uncertainty in the end systolic volume (ESV) measurement of normal rats

Figure 2.31(c) : Comparison for the uncertainty in the EF measurement for three different time duration of normal rats

Figure 2.31(d) : Comparison for the uncertainty in the perfusion homogeneity 
Figure 2.32(a) : Comparison for the uncertainty in the EDV measurement of three different time duration of MI rats

Figure 2.32(b) : Comparison for the uncertainty in the ESV of three different time duration of MI rats

Figure 2.32(c) : Comparison for the uncertainty in the EF of three different time duration of MI rats

Figure 2.32(d): Comparison for the uncertainty in the PH measurement of three different time duration of MI rats 


\section{Glossary of Acronyms}

\begin{tabular}{|c|c|}
\hline ANOVA & ANalysis Of Variance \\
\hline bpm & Beats Per Minute \\
\hline CAD & Coronary Artery Disease \\
\hline CVD & CardioVascular Disease \\
\hline DALY & Disability-Adjusted Life Year \\
\hline $\mathrm{EC}$ & Electron Capture \\
\hline ECG & $\begin{array}{l}\text { ElectroCardioGram - a measurement of the electrical signals of the heart } \\
\text { produced during cardiac contraction }\end{array}$ \\
\hline EDV & End Diastolic Volume \\
\hline $\mathrm{EF}$ & Ejection Fraction \\
\hline ESV & End Systolic Volume \\
\hline FBP & Filtered Back Projection \\
\hline $\mathrm{Hg}$ & Mercury \\
\hline HLA & Horizontal Long Axis plane of the heart \\
\hline HR & Heart Rate \\
\hline IT & Isomeric Transition \\
\hline LAD & Left Anterior Descending - one of 3 major arteries of the heart \\
\hline LCX & Left CircumfleX - one of 3 major arteries of the heart \\
\hline LV & $\begin{array}{l}\text { Left Ventricle / Left Ventricular - one of four chambers of the heart that } \\
\text { pumps blood throughout the body }\end{array}$ \\
\hline LVEF & Left Ventricular Ejection Fraction \\
\hline MI & Myocardial Infarct \\
\hline ML-EM & $\begin{array}{l}\text { Maximum Likelihood Expectation Maximization - an image reconstruction } \\
\text { algorithm }\end{array}$ \\
\hline OS-EM & $\begin{array}{l}\text { Ordered Subsets Expectation Maximization - an image reconstruction } \\
\text { algorithm }\end{array}$ \\
\hline
\end{tabular}




$\begin{array}{ll}\text { PH } & \text { Perfusion Homogeneity } \\ \text { PMT } & \text { PhotoMultiplier Tube } \\ \text { PSF } & \text { Point Spread Function } \\ \text { RCA } & \text { Right Coronary Artery - one of } 3 \text { major arteries of the heart } \\ \text { RR } & \text { Respiratory Rate } \\ \text { SA } & \text { Short Axis plane of the heart } \\ \text { SPECT } & \text { Single-Photon Emission Computed Tomography } \\ \text { Tc } & \text { Technetium } \\ \text { Tl } & \text { Thallium } \\ \text { VLA } & \text { Vertical Long Axis plane of the heart } \\ \text { WHO } & \text { World Health Organization }\end{array}$




\section{Chapter 1}

\section{Introduction}

\subsection{Cardiac Disease and SPECT}

\subsubsection{Around the World}

Cardiovascular diseases (CVD) refer to a group of disorders of the heart and blood vessels. CVD encompasses coronary heart disease, cerebrovascular disease, peripheral arterial disease, rheumatic heart disease, congenital heart disease, raised blood pressure, deep vein thrombosis and pulmonary embolism. Almost 17 million people die every year from heart disease and stroke and these account for almost one-

- third of all deaths around the world. According to The Atlas of Heart Disease and Stroke, (Mackay, J. 2004) published by the World Health Organization (WHO), CVD is the principal cause of global death and disability. Moreover, the number of fatalities is projected to increase to over 24 million per year by the end of 2030 . The largest percentage increase is estimated to take place in the Eastern Mediterranean Region while the largest increase in number of deaths is projected to occur in the South-East Asia region. Almost eighty percent of deaths from CVD occur in low and middle income countries, but high-income countries are not immune. Despite all the advancement of technology as well as the improvements in health care facilities in the USA, one in four men and one out of three women still die in the USA within in a year of a recognized first heart attack ( Mackay, J. 2004).

Some of the principal risk factors for CVD are tobacco use, physical inactivity, heavy alcohol use, and an unhealthy diet, but more than 300 risk factors associated with CVD have been identified so far. Cardiovascular risk factors also 
include mental ill-health, psychological stress, and diabetes mellitus. CVD does not respect national borders, but the prevalence of different risk factors and the demographics of the disease vary between developing and developed countries. In the developed countries, the major risk factors are identified as tobacco use, alcohol use, high blood pressure, high cholesterol and obesity whereas in the developing countries CVD is attributed more to the nutritional and infectious problems of those countries. Socioeconomic status has been positively related to CVD in the developing countries with coronary heart disease being more common in the rich socioeconomic status group. In the developed countries, such as Canada, the United States, and the United Kingdom, the past couple of decades have revealed a different picture suggesting a negative relationship between the socioeconomic status and coronary heart disease. In Canada, children from poor families are twice as likely to be obese as children from rich families (Mackay, J. 2004) and so also have an increased prevalence of CVD associated with this risk factor. Overall death rates from coronary heart disease have decreased in North America and Western Europe whereas mortality is increasing in developing and low and middle income countries with men and women being equally vulnerable to CVD.

One of the measures of global burden of CVD is the disability-adjusted life years (DALYs) lost which is the number of healthy life years a person loses due to CVD as opposed to the resulting deaths. Ten percent of the DALYs lost in low and middle income countries are due to CVD whereas the ratio is higher in high income countries, almost $18 \%$. Moreover, the global burden of the coronary heart disease is projected to increase to 82 million DALYs in 2020. According to the WHO, almost 3.8 million men and 3.4 million women die every year from coronary heart disease. While coronary heart disease is decreasing in developed countries it is increasing 
gradually in developing and transitional countries. More than $60 \%$ of the global burden of coronary heart disease occurs in developing countries (Mackay, J 2004).

\subsubsection{Around Canada}

Heart attacks are one of the leading causes of illness and death in Canada (Statistics Canada 2010). It is estimated that 1.6 million Canadians are living with CVD of some sort (Wielgosz, A. 2009). Almost $15 \%$ of the age group from 65 to 74 years are reported to have heart disease while the percentage rises to $22.9 \%$ for the age group over 75. Almost 67,000 Canadians were reported to be hospitalized with a heart attack in 2008-2009 (Berthelot, JM. 2010). Another report, Health Indicators 2010, divided the Canadian population into five neighbourhood income levels and found that the people living in the least-affluent areas are $37 \%$ more vulnerable to heart attack than those living in the most-affluent areas (Berthelot, JM. 2010). However, there is some progress being made in reducing mortality from CVD. It also reported that there is a decline in the rates of in-hospital mortality and readmission after heart attacks across Canada. The rate of in-hospital death following a heart attack decreased from $10.3 \%$ in $2005-06$ to $8.9 \%$ in $2008-09$ while the rates of readmission dropped from $6.2 \%$ in 2005-06 to 4.7\% in 2008-09. The report 2009 Tracking Heart Disease and Stroke in Canada (Wielgosz, A. 2009) reveals that nine out of ten Canadians over the age of 20 have at least one risk factor for CVD while four out of ten have three or more risk factors. The report also predicted an increase in the number of people with CVD in the future as a result of the increased rate of obesity and of diabetes and the increasing age of the population.

CVD was the second largest contributor to the total health costs in Canada in 2000 and amounted to $\$ 22.2$ billion which included direct health care costs as well as the indirect costs resulting from the loss of economic productivity. Moreover, CVD 
was also responsible for the highest proportion of days in hospital compared to other health problems.

\subsubsection{Role of SPECT Imaging}

Accurate diagnosis and evaluation of the extent of disease or disease progression is essential to appropriate and effective disease management. Medical imaging plays a vital role in modern medical practice because an early and appropriate diagnosis leads to a better treatment strategy and better treatment output. Various modalities measure and visualize different characteristics of the subject under investigation based on the physical phenomena that are being used to obtain the necessary imaging information (Webb, S. 1988; Cho, ZH. 1993). Single-photon emission computed tomography (SPECT) is a non-invasive medical imaging modality which allows us to visualize the functional information of the organ under investigation by using a gamma camera to collect the gamma rays that are emitted from within the patient. Clinical applications of SPECT include the imaging of heart, brain, kidney, bone, liver and lung.

One of the most important clinical uses of SPECT is for the study of myocardial perfusion or blood flow to the tissues of the heart muscle. This test is commonly known as a rest/stress study and is used to assess coronary artery disease and heart muscle damage due to infarction. The common practice is to study the blood flow to the heart under both resting and stress conditions. Stress conditions are created by exercise on treadmill or bicycle, or by drug injection. In a healthy heart, the myocardial blood flow is the same in all coronary arteries under both stress and rest conditions. As a result, when the perfusion tracer is injected into the subject, there will be a homogeneous uptake in all regions of the heart. Under stress conditions there will be an increase of blood flow over baseline. If there is disease such as 
atherosclerosis in the arteries of the heart, flow is reduced which creates heterogeneity in the regional myocardial blood flow image (Wackers FJ. 1994). During the last decade, there have been advances made in nuclear medicine to increase the diagnostic and prognostic value of myocardial perfusion imaging using clinical SPECT (Clark, A. N. 2005). One of the most important of these is gated SPECT. Gated SPECT is the acquisition method of SPECT where the ECG (electrocardiogram) is used to guide the acquisition. Gated SPECT produces several SPECT images corresponding to different phases across the cardiac cycle.

Gated SPECT is used to obtain the diagnostic and prognostic information of the patient who has known or suspected coronary artery disease. With myocardial perfusion gated SPECT imaging, cardiac functions like left ventricular volumes (EDV and ESV) and ejection fraction are derived and it has been demonstrated that they are reproducible and accurate (Sciagra, R. 2007). These functions are able to detect severe coronary artery disease, assess the physiological importance of stenosis, and also predict disease outcomes based on observed perfusion abnormalities (Sciagra, R. 2007). SPECT can also evaluate the efficacy of antianginal drugs, modification of risk factors and therapeutic techniques (Clark, AN. 2005). With all these considerations, gated SPECT is presently considered as an ideal imaging technique for diagnosis and prognosis of cardiovascular diseases in the field of nuclear medicine (Sciagra, R. 2005).

\subsubsection{How it Works - Tracer Technology}

SPECT is based on the imaging of radiopharmaceuticals. A radiopharmaceutical is a radioactive substance which is used for diagnosis or therapeutic treatment. For diagnostic imaging, radiopharmaceuticals are used in trace 
quantities such that their pharmacologic effect is minimal. Thus diagnostic radiopharmaceuticals are commonly referred to as a radiotracer or even more simply as just a tracer. The radiotracer is made with a radionuclide coupled to a drug/pharmaceutical with a target in the body (Bruyne, SD. 2009). The success of the radiopharmaceutical depends on both the drug and radionuclide. While making a radiopharmaceutical, the pharmaceutical is selected first based on its special localization within the patient. Ideally, it should accumulate preferentially based on the function of interest such as blood flow. There should also be little interference from accumulation in other 'background' tissues. A radionuclide is then chosen which is attached to the pharmaceutical. The radionuclide should emit a reasonable radiation after the administration of the drug inside the body and should be detected easily by the radiation detector. Typically, the isotopes used in SPECT imaging emit photons (gamma rays) with energies between 30 and $300 \mathrm{keV}$ (Saha, GB. 2003). This energy range is preferred because below $30 \mathrm{keV}$ too large a percentage of the photons is absorbed by tissues and not detected by the detector and above $300 \mathrm{keV}$ the photons are not collimated well with the common collimators. This gamma ray emission can occur either by internal transition of the nucleus or by the decay of a daughter nuclide produced by electron capture.

Some commonly used isotopes for SPECT and their properties are shown in Table 1.1 (Rowland, DJ. 2008; Saha, GB. 2003). Some common radiotracers used in cardiac SPECT imaging are shown in Table 1.2 (Saha, GB. 2003). 
Table 1.1: Common SPECT radionuclides used for imaging (Rowland, DJ 2008;

Saha, GB. 2003)

\begin{tabular}{|c|c|c|c|}
\hline Radionuclide & $\begin{array}{c}\text { Decay } \\
\text { Mode } \\
(\%)\end{array}$ & $\begin{array}{c}\text { Physical half- } \\
\text { life }\end{array}$ & $\begin{array}{c}\text { Principle } \boldsymbol{\gamma} \text {-ray energy } \\
(\mathbf{k e V})\end{array}$ \\
\hline${ }^{99 \mathrm{~m}} \mathrm{Tc}$ & IT (100) & 6.02 hours & 140.5 \\
\hline${ }^{111} \mathrm{In}$ & $\mathrm{EC} \mathrm{(100)}$ & 2.80 days & 172,245 \\
\hline${ }^{123} \mathrm{I}$ & $\mathrm{EC} \mathrm{(100)}$ & 13.22 hours & 159 \\
\hline${ }^{131} \mathrm{I}$ & $\beta^{-}(100)$ & 8.03 days & 364 \\
\hline${ }^{201} \mathrm{Tl}$ & EC (100) & 3.04 days & $\begin{array}{c}68 \text { to } 80 \mathrm{keV} \mathrm{Hg} \mathrm{x-rays,} \\
135(2 \%) \text { and } 167(6 \%)\end{array}$ \\
\hline
\end{tabular}

IT: Isomeric Transition, EC: Electron Capture 
Table 1.2: Commonly used radiopharmaceuticals in cardiac SPECT (Saha, GB.

2003)

\begin{tabular}{|c|c|}
\hline Radiopharmaceuticals & Uses \\
\hline${ }^{99 \mathrm{~m}} \mathrm{Tc}-$ Sestamibi & $\begin{array}{l}\text { 1. Myocardial perfusion abnormality detection. } \\
\text { 2. Myocardial ischemia and infarct. } \\
\text { 3. Evaluation of myocardial functions. } \\
\text { 4. Detection of breast tumor. } \\
\text { 5. Detection of hyperparathyroidism. }\end{array}$ \\
\hline${ }^{99 \mathrm{~m}} \mathrm{Tc}-$ Tetrofosmin & $\begin{array}{l}\text { 1. Reversible myocardial ischemia detection. } \\
\text { 2. Detection of myocardial infarction. }\end{array}$ \\
\hline${ }^{201} \mathrm{Tl}-\mathrm{TlCl}$ & $\begin{array}{l}\text { 1. Myocardial perfusion imaging (Trace the outline } \\
\text { between ischemic myocardium and infarcted } \\
\text { myocardium). } \\
\text { 2. Myocardial viability } \\
\text { 3. Detection of brain tumor. } \\
\text { 4. Hyperparathyroidism detection. }\end{array}$ \\
\hline
\end{tabular}

In SPECT, when a concentration of radiotracer is incorporated into the body it will distribute according to the properties of the pharmaceutical component, leading to preferential accumulation in specific organs of the body. Comparing a patient's distribution to known normal distributions, we can derive functional and pathological information about the body. Primarily, the aim in clinical imaging is to determine the distribution of the tracer and sometimes how it is changing with time. Emission tomography mostly looks at the uptake of the tracer (or lack of uptake) inside the body.

\subsubsection{Parameters Measured with Cardiac SPECT Studies}

In the evaluation of images of the heart, several different parameters have been shown to be important (Yalcin, H. 2008): left ventricular (LV) ejection fraction (LVEF), end systolic volume (ESV), end diastolic volume (EDV), and perfusion 
homogeneity $(\mathrm{PH})$. These cardiac factors are important because they can determine the functional capability of the heart and thereby provide prognostic information. Reduced LVEF, increased EDV, and decreased perfusion in a region of the heart are all indicators of coronary artery disease (CAD). LV function and volume give an indication of the risk and rate of survival of the patient with defined or suspected CAD. Post stress EF and ESV provide significant information on the severity of perfusion defects and on the prediction of cardiac death (Sharir, T. 2007).

Relative myocardial perfusion measurements provide important information regarding the presence of functionally significant coronary artery disease. As indicated previously, the rest-stress test shows where regions of the heart are no longer able to respond to the demand for increased blood flow under stressed conditions. This information can also be used for distinguishing infarcted or dead heart muscle from ischemic tissue, thereby guiding appropriate intervention and patient management. A normal rest-stress perfusion study has associated with it a very low-likelihood ( $<1 \%$ per year) of major cardiac events like death and heart attack (Iskander, S. 1998).

The first radioisotope method for the assessment of LVEF was the ECG-gated planar equilibrium radionuclide angiography (Sharir, T. 2007). Global left ventricular function can be determined by X-ray angiocardiography and 2D echocardiography, but $3 \mathrm{D}$ approaches like computed tomography and magnetic resonance imaging are now considered more accurate (Vanhove, C. 2002). Myocardial perfusion images of gated single photon emission computed tomography (SPECT) also provide an accurate measure of cardiac function with good precision (Everaert, H. 1996). Several different commercial software packages are available that can calculate LVEF from 
gated SPECT studies. It does not cost additional money or any additional radiation exposure over the perfusion study alone.

The research studies in the 1980's illustrated the prognostic values of resting and exercise values of LVEF (Sharir, T. 2007). At that time, due to the lack of technological improvements, LV volumes could not be measured perfectly. But in the 1990's the use of ECG-gated myocardial perfusion SPECT provided precise and reproducible measurements of LV functions and volumes (Germano, G. 1995; Iskandrian, AE. 1998). A research study using the database of Cedars-Sinai Medical Center (Los Angeles, CA) showed that post stress EF value is the best indicator for the prediction of death from CAD (Sharir, T. 2001).

\subsection{Cardiac SPECT Imaging Physics}

\subsubsection{Clinical SPECT}

SPECT produces a 3D image (tomograph) of the isotope distribution inside the body based on the radiation emitted from injected radiotracers. The radiation is mainly a combination of gamma radiation and x-ray radiation in the case of Tl-201. After injecting the radio-tracer into the patient, the tracer emits gamma rays. One or more gamma camera detectors are then rotated around the patient and gather information on the emitted photons. These data are then reconstructed with software and tomograms or 3D images of the tracer distributions are made.

The physics behind SPECT is basically the physics of the Anger camera. Hal Anger, a renowned scientist at the University of California developed this camera in 1958 (Wernick, MN 2004). Though there have since been many small advances, most of today's gamma cameras still mainly use the basic ideas of Hal Anger's camera. 


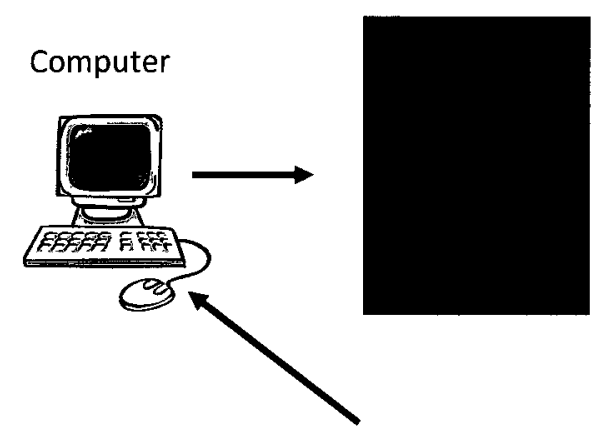

Image of

radioactive

source

distribution

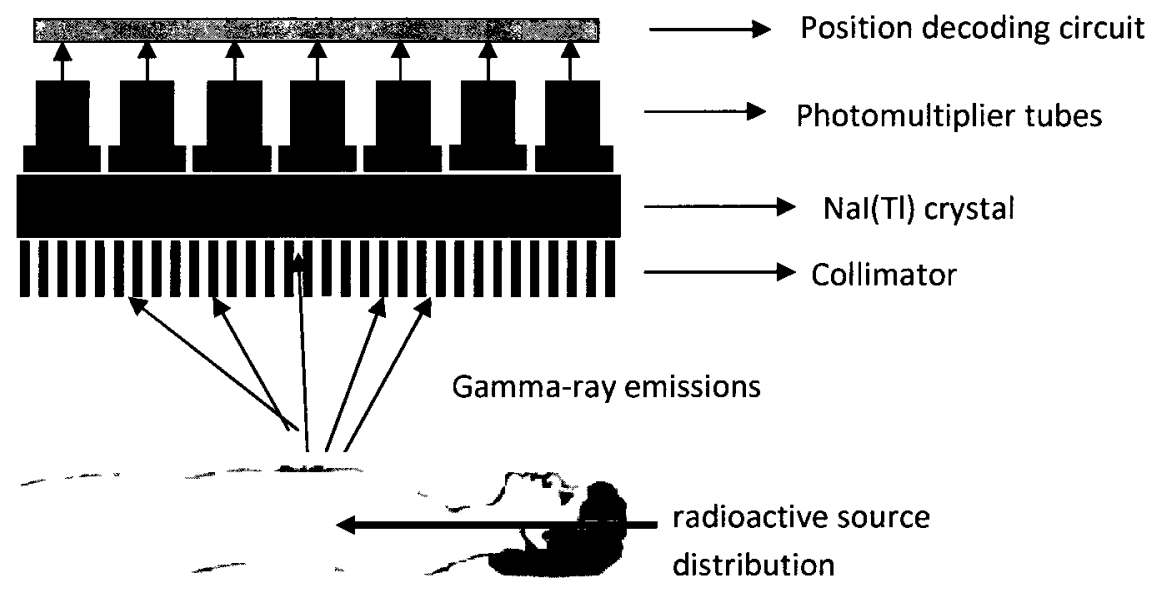

Figure 1.1: Basic components of a typical gamma camera

Figure 1.1 shows the components of a typical gamma camera. At first, a radionuclide is injected to the patient. The radioactivity is then distributed into the organs and gamma photons emitted. The radionuclide emits gamma rays in all directions. Collimators are used to selectively permit only those photons that can reach the detector at a specified angle, typically 90 degrees as in the case of parallelhole collimators (Cherry, SR. 2003). The collimator is generally a lead absorbing plate with holes in it. Photons from the source can pass through the hole and the walls of the holes (the septa) absorb the unwanted photons travelling in the unwanted directions. In general, clinical SPECT systems use parallel-hole collimators. Converging hole collimators like fan-beam and cone-beam collimators can also be used in SPECT systems and provide better detection efficiency than parallel hole 
collimators. But, increased detection efficiency comes at the cost of decreased field of view. So, parallel hole collimators are preferred and converging hole collimators are used only in imaging of small organs like heart or brain.

Those photons that pass through the collimator holes then interact with the scintillation crystal/detector and produce a burst of light. The number of light photons produced is proportional to the energy absorbed from the gamma ray. Gamma cameras use a single $\mathrm{NaI}(\mathrm{Tl})$ detector crystal that can be between $6-12.5 \mathrm{~mm}$ thick and $25-50 \mathrm{~cm}$ in diameter (Cherry, SR. 2003). Most of the general purpose gamma cameras use $9.5 \mathrm{~mm}$ thick crystal. To protect from moisture, the $\mathrm{NaI}(\mathrm{Tl})$ crystal is sealed into a thin $\mathrm{Al}$ casing. On the back surface of the $\mathrm{Al}$ casing, there is an optical glass window which permits the scintillation light to go through to the photomultiplier tubes.

The photomultiplier tube (PMT) is a very sensitive high voltage device which can convert the light signal into an electrical signal and amplify it. On the back surface of the $\mathrm{NaI}(\mathrm{Tl})$ crystal an array of PM tubes is joined with a silicone based grease. To cover the maximum portion of the crystal, the PM tubes are arranged in a hexagonal pattern, in general. Typical PM tube diameter is $5 \mathrm{~cm}$ and most cameras use 30 to 100 PM tubes (Cherry, SR. 2003). To prevent the change in gain due to the earth's magnetic field, PMTs are enclosed in a thin magnetic shielding. Some of the manufacturing companies use a plastic light guide in between the crystal and the PMTs. The light guide can increase the light collection efficiency and reduce the loss of scintillation light from the gap between the crystal and PMTs.

The scintillation crystal absorbs gamma rays from the collimator and converts their energy into light photons. This process is known as scintillation. Here the crystal 
produces long wavelength radiation from short wavelength radiation (ultraviolet photons from gamma photons). $\mathrm{NaI}(\mathrm{Tl})$ crystals can produce one light photon from 30 $\mathrm{eV}$ of gamma energy absorbed. Thus after passing through the collimator, the gamma photons are absorbed by the scintillation crystal and emit light photons. Then the light output is transferred to the PMTs via the light guide. PMTs are the vacuum tube light detectors containing an anode and a cathode. In between the anode and cathode there are several electrodes called dynodes. The cathode or photocathode has a material coated on its inner surface that photoelectrically absorbs light photons that fall on it and then emits photoelectrons. The first dynode has a higher voltage than the cathode and subsequent dynodes each have higher voltages than the previous one. Electrons are accelerated across the electric potential difference and each electron knocks free several secondary electrons when it strikes the next dynode. The electrons are thus propagated from one dynode to another and during this propagation the number of electrons is multiplied. The output is proportional to the number of light photons that initially strike the cathode producing an amplified signal at the end of the PMT.

The interaction of a gamma ray with the crystal produces a flash of light. The point of interaction between the gamma ray and the scintillation crystal can be determined by processing the signal from PMTs. This is basically done by a position decoding circuit. Gamma cameras calculate the weighted position of each of PMT and then calculate the mean position of the incident gamma ray from the weighted positions.

With SPECT imaging, an energy window is often used to exclude unwanted photons. The total signal produced by a gamma detector is proportional to the energy of the incident gamma ray. By using a pulse height analyzer, the energies of incident gamma rays are evaluated and only those which fall in a specific energy range (energy 
window) are accepted (Tsui, BMW. 1996). There are four scenarios for the fate of photons emitted within a patient (Figure 1.2): 1) the photon is photoelectrically absorbed in the detector crystal; 2) the photon Compton scatters inside the patient and is redirected into the detector crystal; 3) the photon Compton scatters in the detector crystal and is only partially absorbed; or 4) the photon is emitted and does not reach the detector crystal because a) it undergoes photoelectric absorption in the patient, or b) is absorbed by the septa of the collimator, or c) its path is not in the direction of the detector. The first scenario represents the desired data. Using a restricted energy window allows us to eliminate many photons from scenario 2 or 3 because the absorbed energy will be different from that of unscattered photons. The position information from these photons is distorted and their presence degrades image quality. A restricted energy window also removes gamma-ray background from different sources (e.g., natural background radiation). 


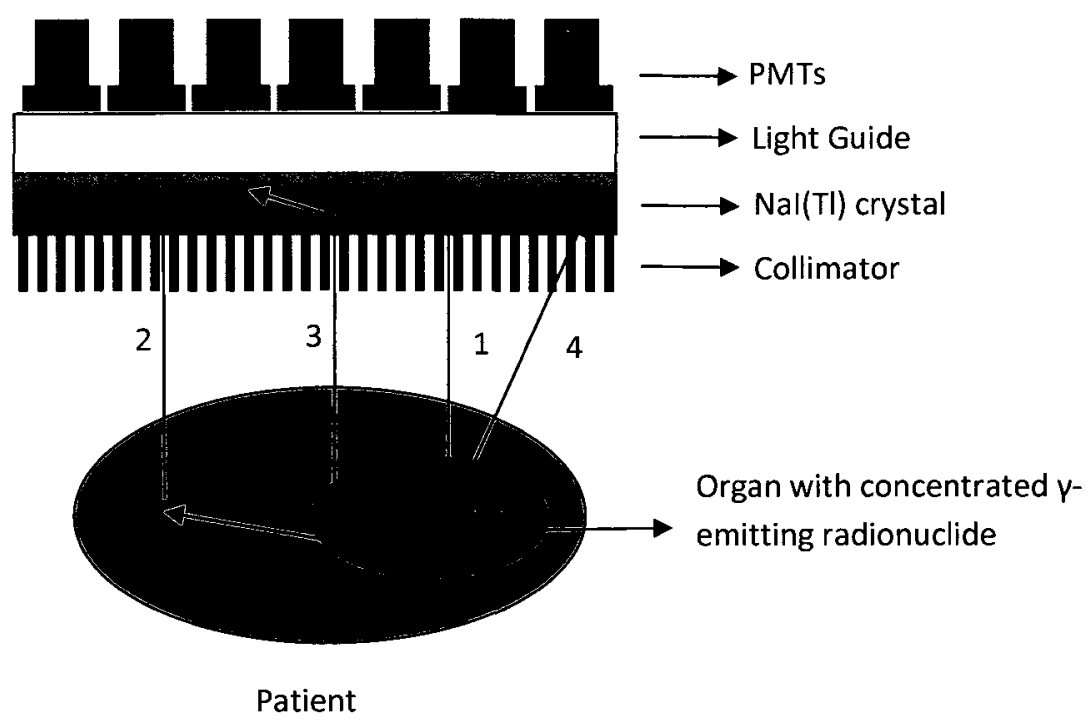

Figure 1.2: Four different types of events that are detected by a gamma camera:

1) Photoelectric absorption, 2) Compton scattered and redirected to the crystal,

3) Compton scattered and partially absorbed and 4) Emitted but does not reach to the crystal.

Single photon emission imaging can be done either in planar mode or tomographic mode. Planar images provide a single 2-dimensional (2D) view of the radionuclide distribution inside the body whereas the tomographic image depicts a volume image (3D image) of the distribution. The volume image is made from multiple 2D images (projections) which are collected at multiple camera positions around the patient. A disadvantage of SPECT is that the head of the detector must be mechanically moved around the patient to collect projection data for tomographic reconstruction. This mechanical motion is slow and increases the time needed to obtain images.

A collimator is required to provide clear images with a gamma camera. However, because all photons travelling in an undesired direction are thrown away, its efficiency is very poor. A solution to this is to build cameras with more than one 
detector (Figure 1.3). More detectors mean more detection efficiency. The disadvantage is increased camera cost. In 2004, almost all of the SPECT systems were with one or two detectors (Wernick, MN 2004).
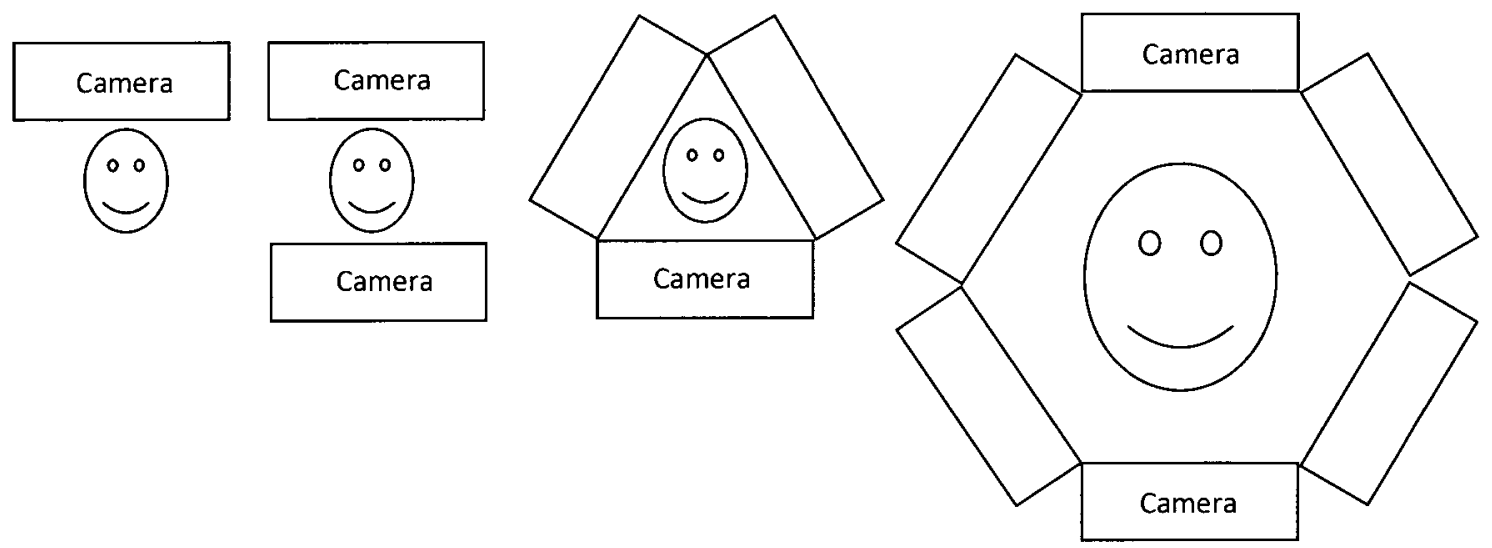

Figure 1.3: Diagram of several SPECT systems with different number of gamma cameras.

\subsubsection{Image Reconstruction}

The aim of image reconstruction in SPECT is to obtain a three-dimensional image from the set of 2-dimensional projections obtained at multiple positions around the subject. Filtered backprojection (FBP) is a common method of image reconstruction (Cherry SR 1987) that is still sometimes used in the clinic. However, more and more, nuclear medicine is moving to iterative methods of image reconstruction and our small animal scanner uses iterative reconstruction exclusively.

A general model of an iterative reconstruction algorithm is shown in figure 1.4. 


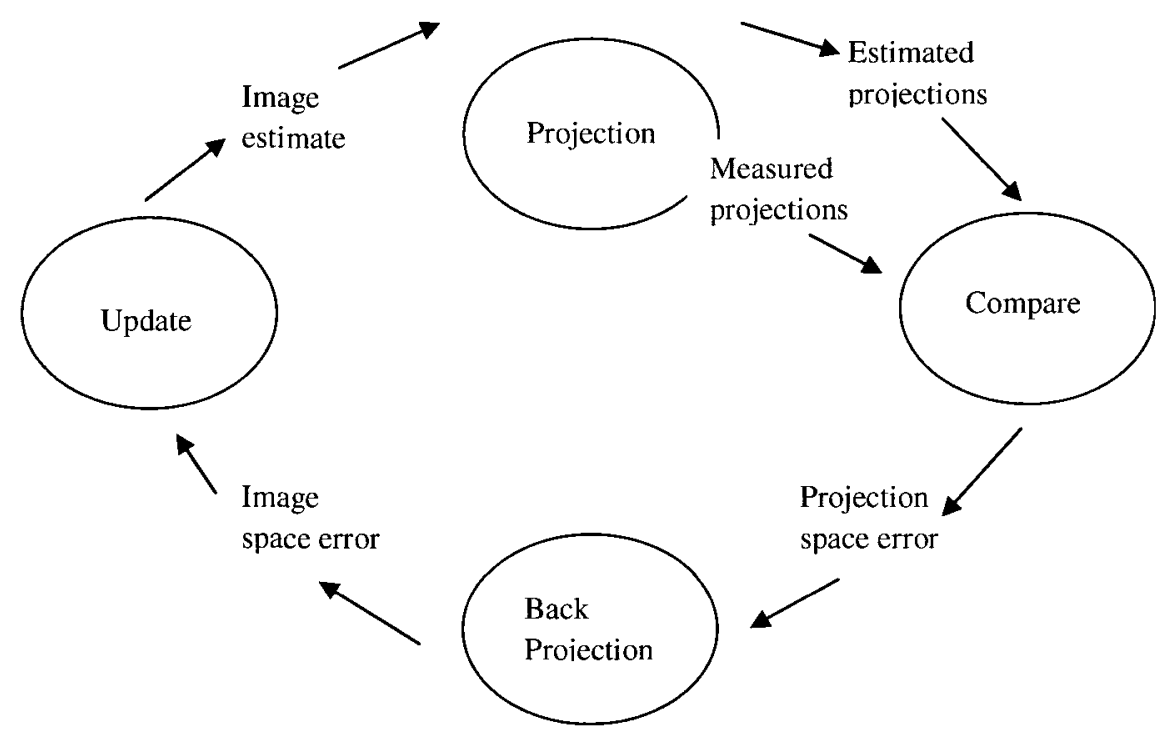

Figure 1.4: Schematic representation of iterative reconstruction method.

The process starts with initial estimation $\hat{f}^{(0)}$ of pixel intensity representing the distribution of tracer in the subject (Wernick, MN. 2004). The estimate is updated as the algorithm progresses and we refer to the current estimate of object tracer distribution as $\hat{f}^{(n)}$, the current image. This is used to calculate a projection value set $\hat{g}^{(n)}$. This $\hat{g}^{(n)}$ is then compared with the actual measured data $g$ to get projection space error values $e_{g}$. These projection space errors are then processed through the back-projection step to create the image space error values $e_{f}$. The image space error values are used to update the image which becomes the new estimate $\hat{f}^{(n+1)}$. The process repeats over and over until it converges or is stopped by the user.

ML-EM algorithm: Maximum likelihood expectation maximization (ML-EM) is one of the leading iterative reconstruction algorithms for SPECT. Equation of MLEM iterative reconstruction is given as equation 1.4.1: 


$$
\hat{f}_{J}^{(n+1)}=\frac{\hat{f}_{J}^{(n)}}{\sum_{\iota^{\prime}} h_{\jmath^{\prime}}} \sum_{l} h_{\iota \jmath} \frac{g_{l}}{\sum_{k} h_{l k} \hat{f}_{k}^{(n)}}
$$

This equation is easy to implement. The most attractive property of ML-EM algorithm is its simplicity. It can be simply implemented with a computer using projection based model. But the main problem with the ML-EM algorithm is that it is slow.

OS-EM algorithm: A modification of maximum likelihood expectation maximization (ML-EM) algorithm is the ordered subsets expectation maximization algorithm (OS-EM) (Hudson HM 1994). The equation of OS-EM algorithm is given by

$$
\hat{f}_{J}^{\text {new }}=\frac{\hat{f}_{J}^{\text {old }}}{\sum_{l^{\prime} \epsilon S_{n} h_{\imath^{\prime}}}} \sum_{l \epsilon S_{n}} h_{\iota j} \frac{g_{l}}{\sum_{l=1}^{N} h_{l l} \hat{f}_{l}^{\text {old }}}
$$

In this algorithm, only the projection bins belonging to the subset $S_{n}$ are used to update the image estimate. Here, a single update is called a subiteration and one pass through all of the subsets is called an iteration. The performance of the OS-EM algorithm is dependent on the organization of subsets. If any of the subsets do not have at least some of the contribution from each pixel then mathematical difficulties will arise: the first summation of the equation (1.4.2) will be zero. Thus, care is taken to ensure that the division of the projection data into subsets is ordered to avoid this problem.

OS-EM has nearly all the attractive properties like ML-EM in addition to a significant increase in speed. Like ML-EM, it also has a built in nonnegativity constraint. Its convergence properties are reproducible and highly predictable 
(Wernick, MN. 2004). The OS-EM algorithm is easy to implement. The main problem of using OS-EM is deciding how to stop the iterations. Running the algorithm to convergence requires a large number of iterations and usually results in a noisy, undesirable image. The practical solution to this problem is simply to halt the reconstruction after a small fixed number of iterations, typically two or three.

In small-animal imaging, one advantage of OS-EM over FBP is fewer artifacts away from the central plane of the pinhole (Vanhove, C. 2000). Compared to the filtered back projection algorithm, OS-EM provides a more accurate image both quantitatively and qualitatively.

For practical use OS-EM is a promising method of reconstruction. It presents the same advantages as ML-EM but requires much less computation time. Hudson said "an order reduction in computations will assist in making real time processing of SPECT and PET data viable with current technologies" (Hudson HM 1994).

\subsubsection{Filtering}

In medical imaging, diagnostic accuracy can be degraded by image noise. Low pass filtering is a way to reduce statistical noise and is usually applied to nuclear medicine images. Many filters are available with several different characteristics and two common examples are the Hann filter and the Butterworth filter.

In nuclear medicine images, noise always dominates the underlying signal at high frequencies (Wernick, MN. 2004). Using a low-pass filter removes the image noise present at high frequencies. This also has the effect of removing the highfrequency component of the object data resulting in a smoother image. The blurring of the image is a disadvantage of applying filters and limits the degree to which 
filtering is applied. Too much filtering can cause the information in the image to be blurred too heavily and lost. Too little filtering can leave too much noise in the image which can also obscure information.

The final choice of imaging parameters is often subjective. The final goal of filtering is an image that physicians find pleasing to view and which they believe best allows them to perform their diagnosis. Figure 1.5 showing three different reconstructed images: true image without filtering (a), noisy image without a filter (b) and with a Hann filter (c) (http:// www. physics. ubc.ca / mirg / home / tutorial / fbp_recon.html). 

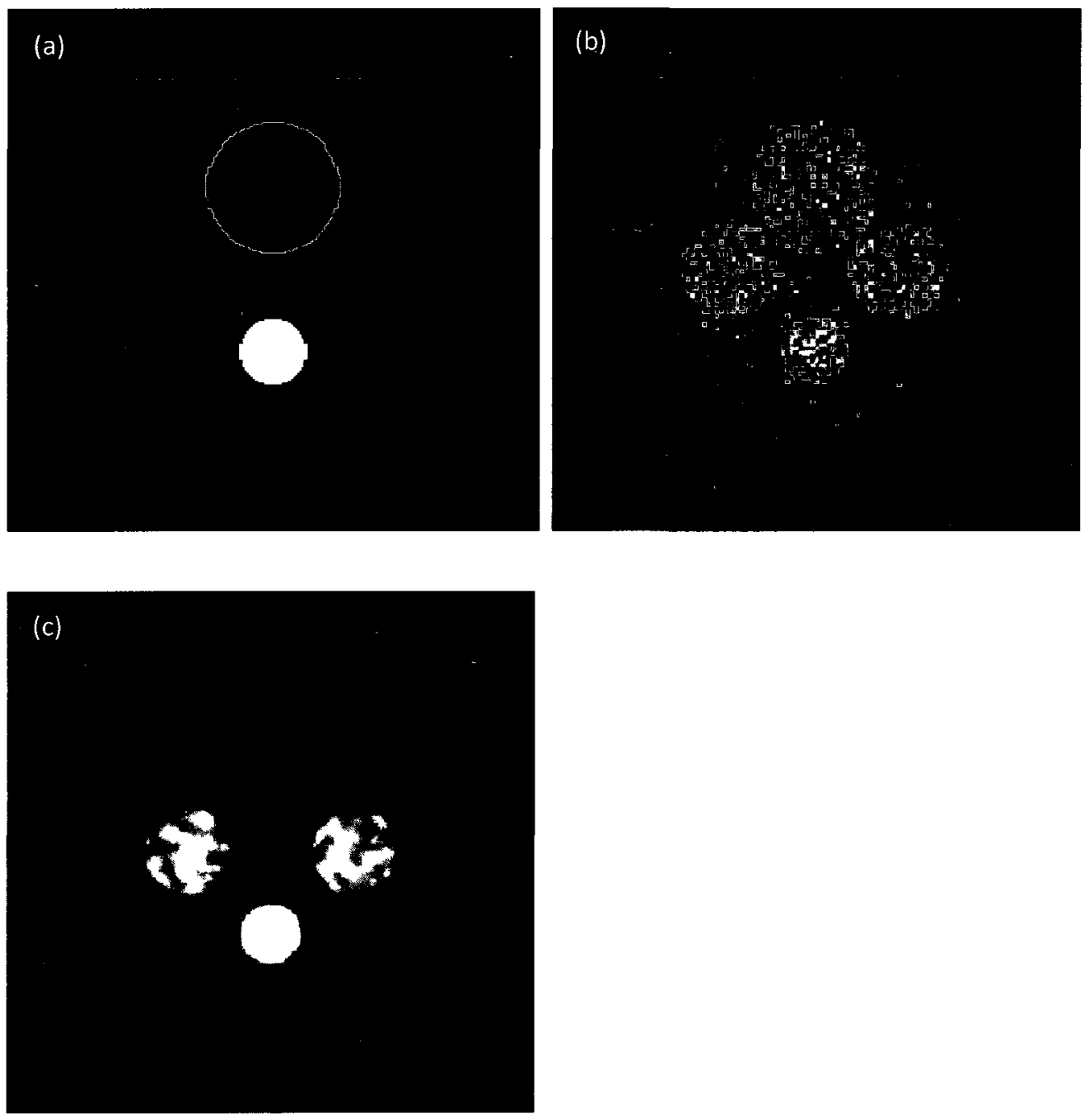

Figure 1.5: Different reconstructed images: (a) True image without a filter, (b)

Noisy image without a filter and (c) With a Hann filter (Reproduced with the permission of the website)

\subsubsection{Analysis of Cardiac Images}

We used 4DM-SPECT to analyze our data. 4DM-SPECT is a commercial software package (Invia Medical solutions, Ann-Arbor, MI) that is commonly used for evaluation of clinical cardiac SPECT studies. This software has been developed to assist this kind of analysis and it is a semi-automatic software program. As soon as we 
have the image of the heart in 4DM-SPECT we need to align the heart along its axes. For this purpose, an ungated image is used (equal to the sum of all of the phases of the ECG-gated image set). After aligning the heart with the axis, the software automatically detects the location of the myocardial wall in all of the ECG-gated images and from this calculates the volume of the heart at diastole (EDV) and systole (ESV) as well as the ejection fraction (EF). The software also automatically creates a polar map (described below) of the heart and divides it according to the standard 17 segment heart model (DePuey, EG. 2006). The uptake in each segment is normalized to the region of maximum uptake in the heart wall. These measurements are then exported to a text file for further processing offline. Because the heart is obliquely positioned within the body, images of the heart are often re-formatted to correspond to the heart axes. The orthogonal axes of the heart are called the horizontal long, vertical long and short axes (American Heart Association 1992). The long axis of the left ventricle goes from the apex, down the center of the ventricle to the base or valve plane separating the ventricle from the atrium. The horizontal long axis is a plane parallel to the long axis that shows the lateral and septal walls of left ventricle. The septal wall is the wall separating the left and right ventricles. The vertical long axis plane is parallel to the long axis and at 90 degrees to the horizontal long axis. Short axis views can be generated by slicing the heart perpendicular to long axis of the left ventricle. Figure 1.6 (adapted from Cerqueira, MD. 2002) shows three different cardiac plane definitions. 

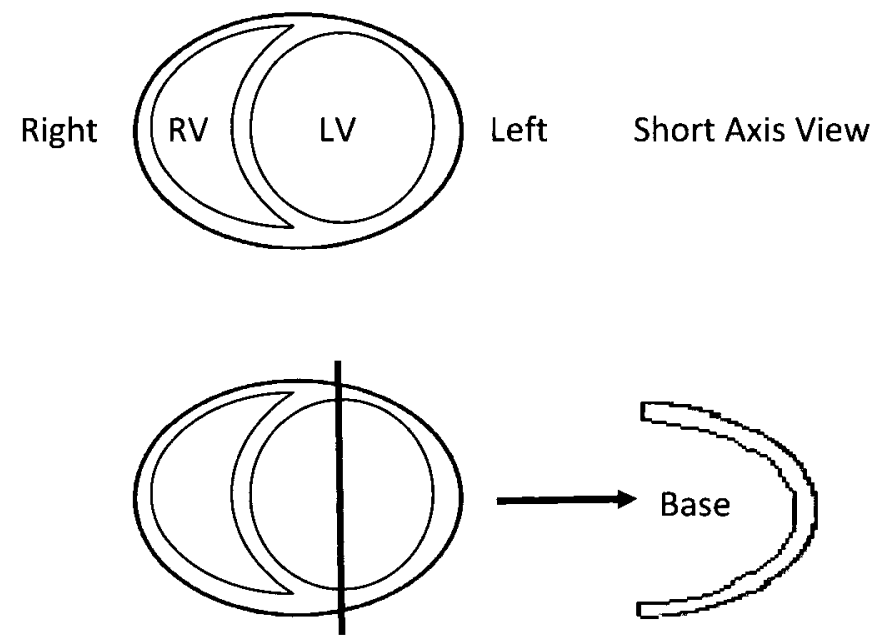

Vertical Long Axis View

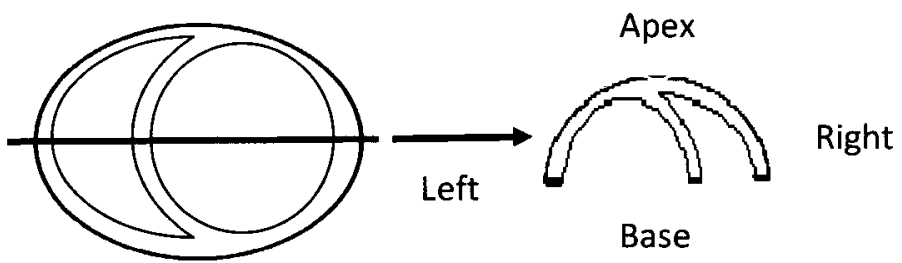

Horizontal Long Axis View

Figure 1.6: Cardiac plane definition for tomographic display. $L V$ refers to the left ventricle of the heart and $R V$ to the right ventricle.

A polar map is a $2 \mathrm{D}$ representation of myocardial activity in the left ventricle. In the polar map, the center represents the apex and the outer portion represents the base of the heart. Figure 1.7 ( Pourmoghadas, A. 2010) shows the polar map with its corresponding 3D heart image. Here, the colour scale shows the activity values. The red portion of the polar map indicate maximum uptake whereas the dark blue portion is the minimum uptake. 

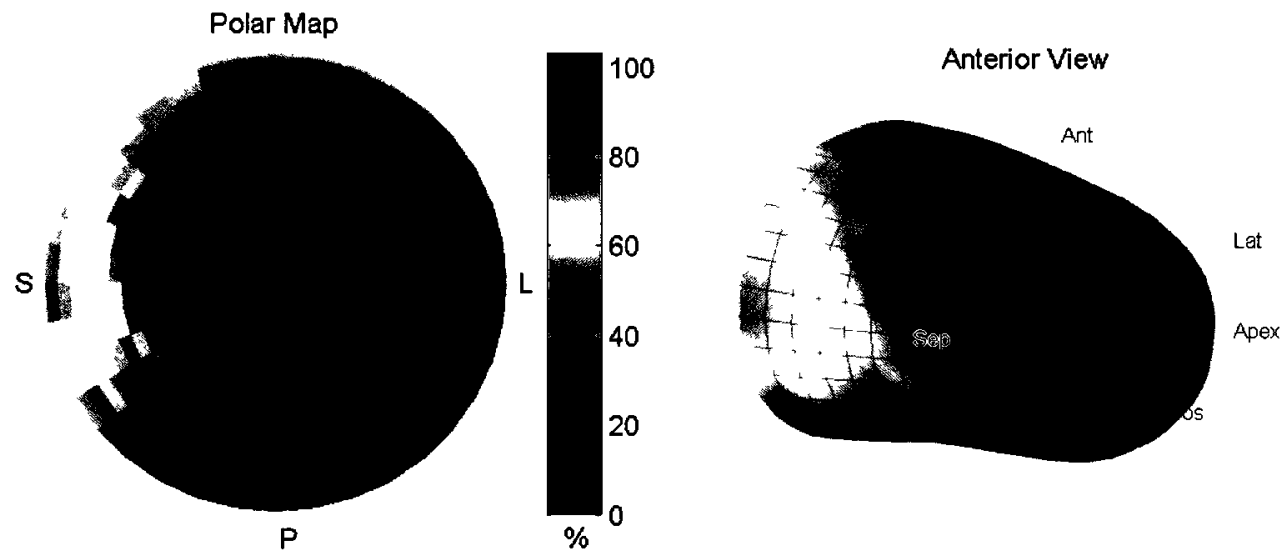

Figure 1.7: Polar map representation with the corresponding 3D LV image. Here S = Septal, $\mathbf{P}=$ Posterior and $\mathrm{L}=$ Lateral. (Reproduced with the permission of Pourmoghadas, A. 2010)

The polar map of LV can be divided into smaller regions to have better idea of regional quantification. A 17 segment model has been used to divide the polar map. The center of this 17 segment model is the apex of the heart and the outermost portion represents the base of the heart. This model is recommended by the American Society of Nuclear Cardiology (ASNC) as the standard model for myocardial imaging (Cerqueira, MD. 2002). This model helps to compare perfusion data from different scans. The name of the 17 segments, as they are in the polar map are shown below (Figure 1.8). 


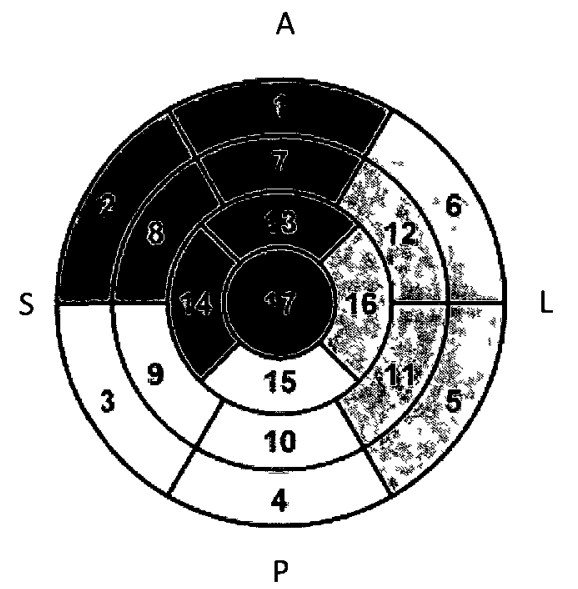
1. Basal Anterior
2. Basal Anteroseptal
3. Basal Inferoseptal
4. Basal Inferior
5. Basal Inferolateral
6. Basal Anterolateral
7. Mid Anterior
8. Mid Anteroseptal
9. Mid Inferoseptal

10. Mid Inferior

11. Mid Inferolateral

12. Mid Anterolateral

13. Apical Anterior

14. Apical Septal

15. Apical Inferior

16. Apical Interal

17. Apex

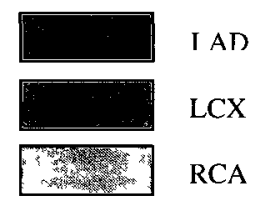

Figure 1.8: 17 segment model of left ventricle $(\mathrm{LV})$. In this figure, A stands for anterior, $S$ stands for septal, $L$ stands for Lateral and P stands for posterior. The colour scheme indicates the territories of the three major coronary arteries.

\subsection{Micro SPECT}

\subsubsection{Rat Model of Cardiac Disease}

In this thesis we have focused on the measurement of uncertainty in the heart of the rat. The rat was chosen because it is a very popular model for the study of human cardiac diseases. The use of animal models in cardiovascular research has allowed the study of cardiovascular disease in the early stages, as well as the 
exploration of the mechanisms of the pathogenesis of cardiovascular disease. Moreover, the animal models have allowed investigating the effects of drug intervention in cardiovascular disease (Doggrell SA 1998). For almost 150 years the laboratory rat (Rattus norvegicus) has been used as an animal model in different areas of research including physiology, pharmacology, toxicology, immunology and neoplasia (Jacob, HJ. 1999). Moreover, in case of cardiovascular research the rat has been used as the animal model of choice for more than a century (Aitman, TJ. 2008). Hypertension, cardiac hypertrophy and heart failure, which are the most common pathophysiological changes in human cardiovascular system, have been successfully reproduced in rat models (Doggrell SA 1998). A lot of experimental, genetic, and transgenic rat models for cardiovascular diseases have been developed. Some of the most frequently used experimental rat models include models for systemic hypertension, models for pulmonary hypertension, models for myocardial infarction, models for cardiac hypertrophy, and models for stroke. Among the experimental models for systematic hypertension the desoxycorticosterone acetate /salt hypertension, nitrous oxide blockade, renovascular hyptertension and angiotensin II infusion are frequently used whereas in the case of models for myocardial infarction, the coronary artery ligation model is the extensively used one. Spontaneously hypertensive rats, Milan hypertensive rats, Lyon hypertensive rats, New Zealand hypertensive rats are some of the frequently used genetic rat models for systematic hypertension. The Fawn-hooded rats model is the commonly used model for genetic models for pulmonary hypertension while the spontaneously hypertensive heart failure rat is the one for genetic models of heart failure. 


\subsubsection{Role of Micro SPECT Imaging}

Molecular imaging entered a new era with the sequencing of the human genome in 2001 and the sequencing of the mouse genome (Rowland, DJ 2008) in 2002. The sequencing of these two genomes made a big impact on molecular imaging as it demonstrated the similarity of human and mouse genomes. This similarity allows many genetic manipulations in the mouse that can directly simulate human diseases (Rowland, DJ 2008). This has greatly expanded our ability to use small-animal models to explore the origin and development of human diseases and also to evaluate better new diagnostic tools such as novel radio-pharmaceuticals and new treatment methods (Cherry, SR 2004).

Just as with humans, imaging animal models can provide important in vivo information about the diseases. As the sizes of small animals are much smaller than humans, thus small animal imaging needs higher spatial resolution than does clinical SPECT. This has led to the development of micro-SPECT (Peremans K 2005). It allows the functional imaging of the heart inside small animals. Micro-SPECT plays an important role in molecular imaging because of its high sensitivity and the ability to readily translate the results of this basic research into clinical care.

There are advantages of micro-SPECT over other modalities. Firstly it has a high spatial resolution which can measure as small as 200 microns (Meikle SR 2005). Secondly, there are many clinical tracers already widely available for evaluating disease that can be used in small animals, like Tc-99m-tetrofosmin. Because these tracers are based on long-lived or generator-produced radio-isotopes, they are readily available to a broad distribution of researchers. Additionally, some SPECT isotopes have half lives of 3 days or more which makes it possible to study slow biological 
processes. Furthermore, nuclear medicine technology is extremely sensitive, allowing detection of very small concentrations of injected tracer (Levin, CS. 2005). Finally, the large number of clinical SPECT scanners in Canada makes it easier to pass any improvement in micro-SPECT research rapidly into clinical care.

\subsubsection{Physics of MicroSPECT}

Micro SPECT systems have been developed to image small animals. In order to image small animals the scanner requires very high spatial resolution. The spatial resolution of clinical SPECT is not good enough to image small animals. For example, the reconstructed image resolution of clinical systems is $10 \mathrm{~mm}$ or more, but the walls of the heart in mice and rats is $1-2 \mathrm{~mm}$ thick. Table 1.3 shows the comparison of scanner performance characteristics among clinical and preclinical SPECT (Rowland, DJ. 2008; Jansen FP 2007).

Table 1.3: Performance characteristics of clinical and preclinical SPECT

\begin{tabular}{|c|c|c|}
\hline & Clinical SPECT & Preclinical SPECT \\
\hline Sensitivity & $0.01 \%-0.03 \%$ & $\sim 0.3 \%$ \\
\hline Resolution & $\sim 10 \mathrm{~mm}$ & 0.5 to $2 \mathrm{~mm}$ \\
\hline Field of View & $\sim 50 \mathrm{~cm}$ & $\sim 8 \mathrm{~cm}$ \\
\hline
\end{tabular}

Micro SPECT systems address the resolution needs mainly by using pinhole collimation, compared to the parallel-hole collimation used in most clinical cameras (Rowland, DJ. 2008; King, MA. 2002; Meikle, SR. 2005; Weber, DA. 1999). Pinhole collimators produce a magnified image of the object so we can have a better spatial resolution image. On the other hand, a pinhole collimator requires that all accepted 
photons pass through a single pinhole which is typically only $0.5-2 \mathrm{~mm}$ in diameter. This reduces the number of photons that can reach the detector and as a result the sensitivity is usually much less than with a parallel-hole collimator.

Spatial resolution is characterized by the full-width-at-half-maximum of the point response function which means the full width at the half of the maximum of the image of an extremely small point source of radiation (Cherry SR 1987).

$a=$ Distance from the detector to the pinhole,

$b=$ Distance from the pinhole to the source,

$d_{e}=$ Effective diameter of the pinhole.

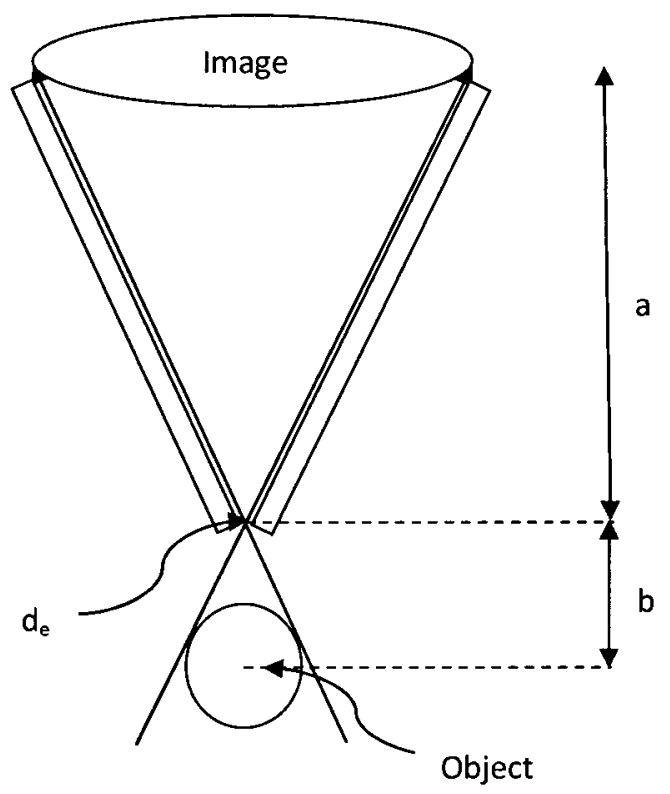

Figure 1.9: Schematic diagram of a single pinhole collimator, imaging an object, showing how it produces a magnified image of the object.

In reference to figure 1.9 , the system resolution of the SPECT system can be written as (Ishizu, K. 1995)

$$
R_{s}^{2}=R_{g}^{2}+(b / a)^{2} R_{i}^{2}
$$




$$
\begin{aligned}
& R_{g}=\frac{d_{e}(a+b)}{a}=d_{e}+d_{e}(b / a) \\
& S=k \cdot d_{e}^{2} / b^{2}
\end{aligned}
$$

Here $R_{s}$ is the system resolution, $R_{g}$ is the geometric resolution of the pinhole, $R_{i}$ is the intrinsic resolution of the detector, $a$ is the distance from the detector to the pinhole, $b$ is the distance from the pinhole to the source, $k$ is a constant, $d_{e}$ is the effective diameter of the pinhole and $S$ is the system sensitivity.

From the above equations we can see that, if the source is very close to the pinhole, then the system resolution would be just the effective diameter of the pinhole and this means the resolution would be in the millimeter range. Again, if the source is a long way away from the pinhole then the resolution would be degraded. As small animals are much smaller than the patient (human) so it is easier for a pinhole to get closer to the animal/source and obtain better resolution. This is why pinhole collimator is mostly used for small animal imaging but not for clinical cases. The drawback of using pinhole collimation is the poor sensitivity of these collimators, but scientists have found ways to minimize this problem. One way is using multiple detectors. Another way is using multiple-pinhole collimators which project multiple overlapping images of the object onto the detector (Meikle SR 2005). Both of these approaches were applied in the commercial small animal SPECT system, NanoSPECT from Bioscan Inc. (Washington, DC) that we used in our experiment: it uses four detectors, each with a 9-pinhole collimator. This system has comparatively high sensitivity (1100 cps/MBq) and sub-mm resolution (Forrer, F. 2006).

Small animal imaging uses the radiotracers which emit photons in the range of 25-250 $\mathrm{keV}$ (King, MA. 2002). This is because the photons in this range have a high enough energy to have a good chance to escape from the animal and yet have a low enough 
energy to be absorbed by the detector. For small animal imaging we can use isotopes with lower energies down to $25 \mathrm{keV}$, rather than stopping at about $50-60 \mathrm{keV}$, as is usually done in the clinic. The lower limit is possible because with small animal imaging the attenuation is much less than with humans.

\subsubsection{Sources of Uncertainty}

In this thesis, our primary goal is to measure the uncertainty in cardiac imaging with microSPECT. Many factors are responsible for inaccuracy and uncertainty in micro SPECT and clinical SPECT.

Anatomy and Physiology: The outcome of SPECT depends on the activity distribution inside the patient body (Tsui BMW 1994). So SPECT imaging will depend on the physiology and anatomy of the patient. This leads to considerable variability when comparing scans between different patients. With small-animal imaging, because the rats are much more similar to one another than are humans typically, there should be less variability between animals, reducing inter-subject variance.

Tracer redistribution: If the time of acquisition is long enough, then the changes over time of the activity of the patient's specific organs may cause image artifacts (Bok BD 1987; Links JM 1991). Tl-201 is known to redistribute over time in humans. This is the basis of the stress-redistribution acquisition protocol commonly used (DePuey, EG. 2006). In rats, we have observed that T1-201 rapidly washes out of the heart such that very little is left in the myocardium after an hour. Changing tracer distribution can degrade image quality and increase uncertainty because reconstruction algorithms assume the distribution is unchanging during acquisition. 
Patient motion: Patient motion during scanning and physiological motion like breathing or the heart beating also result in lower accuracy and may affect diagnosis. Unlike humans, rats are anaesthetized for imaging, reducing the likelihood of voluntary patient motion and its associated artifacts and uncertainties. However, the rat metabolism is very fast compared to humans. Although the rats are anaesthetized and the heart rate is less than normal, the rat heart rate can still be more than $300 \mathrm{bpm}$ (beats per minute). It is difficult to measure very fast heart rate of rodents for ECG gating and the heart rate may also vary from study to study. This fast metabolism and fast heart rate can lead to poor gating signal and degrade image quality.

Attenuation of Photons: Attenuation means the loss of counts or photons. This is due to the absorption or the scattering of photons by the patient body. Photon attenuation depends on photon energy, the tissue thickness travelled by the photon and on the medium (Johns, HE 1983). So in SPECT, attenuation depends on the patient size, the location of the source of the activity and the organ to be imaged. The attenuation effect can cause a reduction by $20-25 \%$ of the original photon intensity in case of brain and cardiac studies using Tc99m (Tsui BMW 1994). This attenuation effect results in uncertainty, degrades image quality and affects clinical diagnosis (DePuey EG 1989;Manglos SH 1987; Tsui, BMW. 1992). If an artefact is such that it could be mistaken for a true abnormality, then it can reduce the confidence of a physician who is making a diagnosis and thus introduce uncertainty. This aspect is not as relevant for our study as we are taking numerical measures on the image and not evaluating them diagnostically. However, attenuation leads to fewer photons detected. We know that noise is Poisson distributed, so lower signal means higher percentage of noise in the signal which can increase the uncertainty in our measurements. The magnitude of this problem in small-animal imaging will be much 
smaller than for clinical imaging due to the much smaller subject size which leads to much less attenuation.

Attenuation results from the interaction of radiation and matter. One of these interactions is the photoelectric effect. In the photoelectric effect the photon is absorbed by the matter and an electron is ejected from the outer shell of the atom. As a result there will be an ionized atom and the absorption of the photon will occur. This ionized atom then returns to its natural state and emits a lower energy photon. Generally this lower energy photon is absorbed by the body and is not detected by the gamma camera. Photoelectric absorption depends both on the energy of the gamma ray and the atomic number of the attenuation material. For most soft tissues at the energies used in nuclear medicine, the dominant interaction of radiation with matter is not photoelectric absorption but rather Compton scattering.

Scattering of Photons: Compton scattering of photons refers to the interaction of photons with matter which changes the direction of the incident photon and reduces its energy. The scattered photon can be deflected in a direction such that it is passes through the collimator and is detected within the energy window of the camera due to the camera's poor energy resolution. Scattered photons degrade the reconstructed image by lowering image contrast (Jaszczak R J 1980; Jaszczak R J 1985; Jaszczak RJ 1981). By blurring the position of the object boundaries it can make harder to estimate the true size of the object (e.g. the edge of ventricle). Also it could affect observer confidence by making features in the image harder to see. As with attenuation, the amount of object scatter is much less with small animals than with humans due to their much smaller size. Some scatter will also occur within the components of the camera detector itself. The number of accepted scattered photons is reduced by using an appropriate photopeak energy window. 
Detector response function: Spatial resolution is one of the important characteristics of image quality. Geometric response of the collimator is the main component of detector response. As the distance of the source from the collimator increases, the width of the geometric point spread function increases (Metz CE 1980; Tsui, BMW 1988). Septal penetration in parallel-hole collimators or equivalently edge-penetration in pinhole collimators further degrades resolution. As spatial resolution is very important for small-animal SPECT, some form of point-spread function modelling is usually integrated into the image reconstruction to compensate for these losses.

Detection efficiency: The quality of image depends on the number of counts acquired and detection efficiency describes the number of photons counted by the detector. This is the biggest camera source of uncertainty. The collimator-detector of a SPECT system is the main factor defining detection efficiency. A conventional gamma camera uses a parallel-hole collimator for which geometric efficiency is constant as a function of source-to-collimator distance. With a pinhole camera, detection efficiency falls off rapidly with distance from the pinhole. This results in poorer efficiency and correspondingly greater noise and uncertainty in the more distant parts of the image.

The camera itself can also be a source of errors and uncertainties. The PMT converts the light signal into electrical signal, meaning it converts photons into electrons. The numbers of electrons are not exactly the same as the number of photons because of the statistical nature of the process. This produces an uncertainty in the measured energy of the photon. This in turn introduces uncertainty in the measured position of the detected gamma-ray and degrades image quality. Changes in the performance of the camera over time can lead to variation in the accuracy of 
corrections that are applied to the raw data: corrections for detector uniformity, linearity, and center of rotation for example.

Physiological variability of the animal over time may also introduce uncertainty. Changes in animal weight, heart size, heart rate, body temperature, level of anaesthetic, actual changes in infarcts in the case of infarcted animals are some other factors that can affect the reproducibility of measurements. For example, bigger hearts are possibly easier to measure than smaller ones. Lower weight rats have smaller hearts and are more difficult to image. High levels of anaesthetic make the heart beat more slowly and may introduce uncertainty through the accuracy of the ECG-gating.

In our experiment the rat position was supine. From human handling the positioning of the animal may not be same for each of the scanning sessions. This change in the positioning of the animal may introduce variation in the images.

There could be also variation in the processing of the data while doing the alignment of heart before processing into a polar map. Alignment of the heart is done by humans, so the alignment may not be exactly the same each time.

\subsubsection{Need for Measuring Uncertainty in MicroSPECT}

In this thesis we are interested in the measurement of uncertainty of cardiac microSPECT measurements. MicroSPECT allows the repetitive imaging of a single animal. Serial imaging of a single animal can reduce the cost of research significantly and also reduce the number of animals that need to be sacrificed. This could save a huge amount of money and invested time in research every year. This saving is related to the decrease of sample size which is in turn related to the knowledge of 
uncertainty in the measurements. MicroSPECT using multi pinhole collimators leads to the overlapping of images in the projection data. So, the gain of the signal to noise may be reduced because of the ambiguity of the direction of the incident photon. Also the uncertainty influences how far one can trust an observed change. Most applications of microSPECT involve looking for changes in the images, for example, determining if the size of an infarct is smaller after applying a treatment. Knowing the uncertainty in the measurement allows one to distinguish between true changes and simple measurement noise.

Myocardial microSPECT imaging is a complex technology for the detection and assessment of cardiac diseases. Successful operation of this technology depends on the skill and knowledge of both the technologist who acquires the data and the analyst who evaluates the images. A failure can generate an inferior image and produce errors in analysis. Few studies have investigated the reproducibility and the reliability of cardiac microSPECT. Vanhove et al (Vanhove C 2005) studied the reproducibility of cardiac factors using Tc-sestamibi in a rat model. Using 8 normal rats in their study they obtained 2 gated perfusion SPECT images and 2 gated blood pool SPECT images with a single pinhole clinical gamma camera. They calculated nearly $2 \%$ differences between the two acquisitions of their study, averaged over all animals. This study did not distinguish between intra-subject and inter-subject reproducibility and thus may overestimate the uncertainty in serial imaging of a single animal. Another group, Constantinesco et al (Constantinesco A 2005) also performed baseline measurements for left ventricular perfusion, volumes and ejection fraction in mice using gated SPECT. This group did not provide any indication of reproducibility. Our own group has investigated the reproducibility of cardiac measurements in rats with Tc-99m-tetrofosmin. However, the primary emission 
energy, typical injected amounts of radiotracer and corresponding signal-to-noise ratio, and pharmacokinetics of the tracer are very different from those of Tl-201. It is not clear that results from that study could be applied to Tl-201 imaging. I am not aware of any other studies that address reproducibility in cardiac microSPECT imaging, making our study of fundamental importance for the application of this technology.

\subsection{Study Objective}

Gated myocardial single photon emission computed tomography (SPECT) plays an important role in measuring cardiac function. MicroSPECT or small animal SPECT is a non-invasive technique for in vivo measurements of cardiac diseases which is used to develop new methods of therapies and new radiotracers, follow disease progression and determine prognosis.

The objective of our study is to determine the reproducibility of Tl-201 for cardiac functional measurement using microSPECT imaging in a rat model. Myocardial perfusion homogeneity (PH), end diastolic volume (EDV), end systolic volume (ESV) and ejection fraction (EF) are important indices of heart function and can easily be obtained from microSPECT experiments. Rat models are used to obtain these indices. MicroSPECT also permits repetitive imaging of a single animal which allows monitoring of disease progression inside the subject over time. Repetitive imaging can reduce the number of animals to be sacrificed and the cost of research (Vanhove C 2005). Tl-201 is a perfusion tracer which is commonly used in clinical and research studies. During the recent shortage of Tc-99m, Tl-201 provided a potential substitute for Tc-99m-based tracers. Our aim is to establish the intrasubject and inter-subject variability for both normal rats and rats with a surgically 
induced myocardial infarction. In Chapter 2, I discuss the method and results of our experiments. In Chapter 3, I conclude the thesis by providing a broader view of the implications of this research and some of the directions it might take in the future.

\subsection{My Contributions}

There were several components to this project. In the imaging of the rats with the microSPECT system, I was assisted by Julia Lockwood, our imaging technician. The handling of the animals, including injection of the radioactivity was performed by JL. The operation of the scanner itself, and recording of the data, and physiological monitoring of the animals was shared between JL and myself. The surgery performed to generate our myocardial-infarct rat model was done by JL. Once acquisition was finished, I performed all of the image post-processing. This included combining the separately acquired $10 \mathrm{~min}$ scans into $20 \mathrm{~min}$ and $30 \mathrm{~min}$ duration data sets. I then performed all of the image reconstructions using the vendor supplied software. I then transferred these images to our clinical computers for analysis with the commercial software package 4DM SPECT. I performed all of the analysis using this software package. The $4 \mathrm{DM}$ results were transferred offline and I then performed further statistical analysis and comparison of these results.

I also prepared the results of this work for presentation. I presented this work at two separate conferences:

1. Canadian Organization of Medical Physicist (COMP) Conference in June 2010. Med.Phys. 37, 3897 (2010) [abstract].

2. Nuclear Science Symposium and Medical Imaging Conference (NSS/MIC) in October 2010. 2010 IEEE Nuclear Science Symposium and Medical Imaging Conference Abstract Book, 388 (2010) [Abstract]. 


\section{Chapter 2}

\section{Reproducibility of T1-201 for cardiac micro SPECT}

\subsection{Introduction}

Gated myocardial perfusion single photon emission computed tomography is a non invasive tool for in vivo measurements of cardiac function. Rat models of in vivo cardiac function are a valuable tool for studying disease progression and for developing new therapies and radiotracers. ECG-gated micro-SPECT imaging of rat models can provide information on cardiac function. Some important parameters that can be obtained from microSPECT myocardial perfusion images are: end diastolic volume, end systolic volume, ejection fraction and myocardial perfusion homogeneity. In addition, the repetitive imaging of a single animal can decrease the cost of research by increasing statistical power and thereby decreasing the number of animals that need to be sacrificed. A key determinant of statistical power is the uncertainties of the measurements being made. There are many possible factors that can contribute to the uncertainty of imaging rat hearts with microSPECT. Some of these sources of uncertainty include statistical noise, operator variability, movement of animal and the variability in camera electronics. Among them one of the major reasons of uncertainty is the size of the heart. The wall of the heart is nearly $1 \mathrm{~mm}$ thick. The spatial resolution of the camera is similar to the thickness of the heart wall. This can introduce partial volume effects which can affect the reconstructed activity concentrations. As a result the infarct size, ejection fraction and left ventricular volumes may not be accurately measured (Meikle SR 2005). Another important difficulty of serial imaging is the possibility of physiological changes in the animal 
over time. A third is variability in the positioning of the animal for each imaging session. The combined effect of these uncertainties on measures of cardiac function in rat microSPECT imaging is not well understood.

A common tracer used for perfusion studies is T1-201. Thallium-201 imaging is of interest first because it emits at different energies than Tc- $99 \mathrm{~m}$ allowing for dualisotope imaging with other Tc-99m-labelled tracers, and second because it is a cyclotron-produced alternative to Tc-99m which was in short supply during the shutdown of the Chalk River reactor. However, the amount of activity injected with Tl-201 is less than with Tc-99m-labelled tracers due to the increased dose. As the activity is lower, it will produce lower counts and the final image will be degraded. For the same duration of scanning, Tl-201 provides a lower quality image than Tc$99 \mathrm{~m}$-tracers. The lower energy $(70 \mathrm{keV})$ of the primary emission peak also increases the potential influence of attenuation. Attenuation refers to the decrease of the number of detected photons. Thus the final output will be a degraded image. Both factors may alter the precision of microSPECT measurements compared to the more commonly used Tc-99m-based tracers. At present, there are no reports to our knowledge on the uncertainty of microSPECT imaging with Tl-201. Our aim is to find the inter-subject and intra-subject reproducibility of measurements of cardiac function using microSPECT in a rat model. 


\subsection{Methodology}

\subsubsection{Animal Handling}

\subsubsection{Normal Rat}

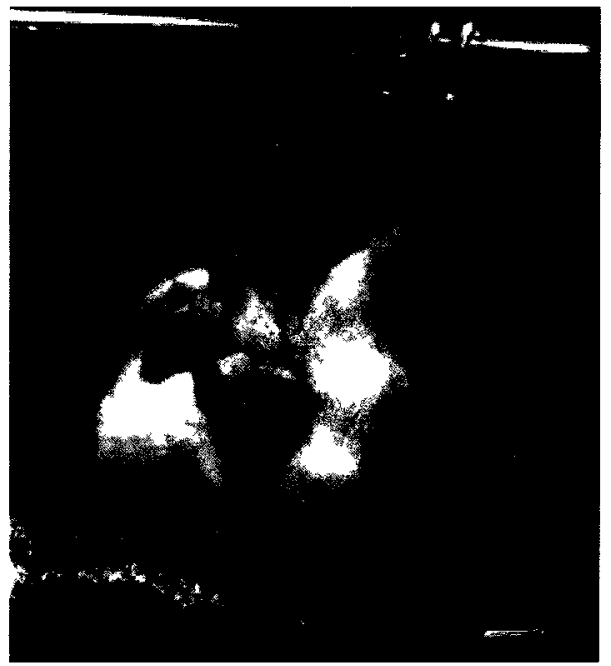

Figure 2.1: Sprague-Dawley Rats - which we were working with.

All of the normal-rat data were collected using 8 normal Sprague-Dawley rats (Figure 2.1). The age of the rats at the start of the study was 7 weeks. The rats were housed 2 rats per cage in a standard rat cage. They were maintained on a 12 hour day/night cycle, meaning that the light was on in the animal room for 12 hours (7 am $-7 \mathrm{pm})$ and then the light was off for another 12 hours (7 pm - $7 \mathrm{am})$. The housing conditions were kept constant during the experiment in order to minimise the variation of their physiological parameters. The rats had free access to water and food before and after the scanning. 


\subsubsection{Myocardial Infarcted Rat}

All of the infarcted-rat data were collected using 8 different Sprague-Dawley rats in which a myocardial infarction was created surgically. At first, the rats were incubated and ventilated using a small animal ventilator. Then the chest was opened at the $5^{\text {th }}$ intercostal space with an incision parallel to the ribs. After spreading the ribs, the heart was exposed and the pericardium was opened. Just beneath the left atrial appendage, the LAD (left anterior descending artery) was visualized as the artery that goes directly to the left ventricular apex. The LAD was ligated with a suture passed through the myocardium and tightened firmly with a knot to create a permanent ligation. Finally, the chest was closed with layered sutures and staples. All the surgeries were performed by two animal technicians (Julia Lockwood and Stephanie Thorn) to maintain the consistency in the surgical model. The rats were allowed to recover for four weeks before scanning was started. Other studies have shown that the remodelling of the heart is normally complete by this time and the infarct size is stable (Fishbein MC 1978). For myocardial infarct rats we maintained the same housing and food/water conditions as for the normal rats.

\subsubsection{SPECT Data Acquisition}

All the animals were scanned with a NanoSPECT/CT scanner (Figure 2.2) from Bioscan (Washington, DC). The scanner had 4 detector heads with 9 pinholes per head for a total of 36 pinholes. The pinhole diameter was $2.5 \mathrm{~mm}$. 


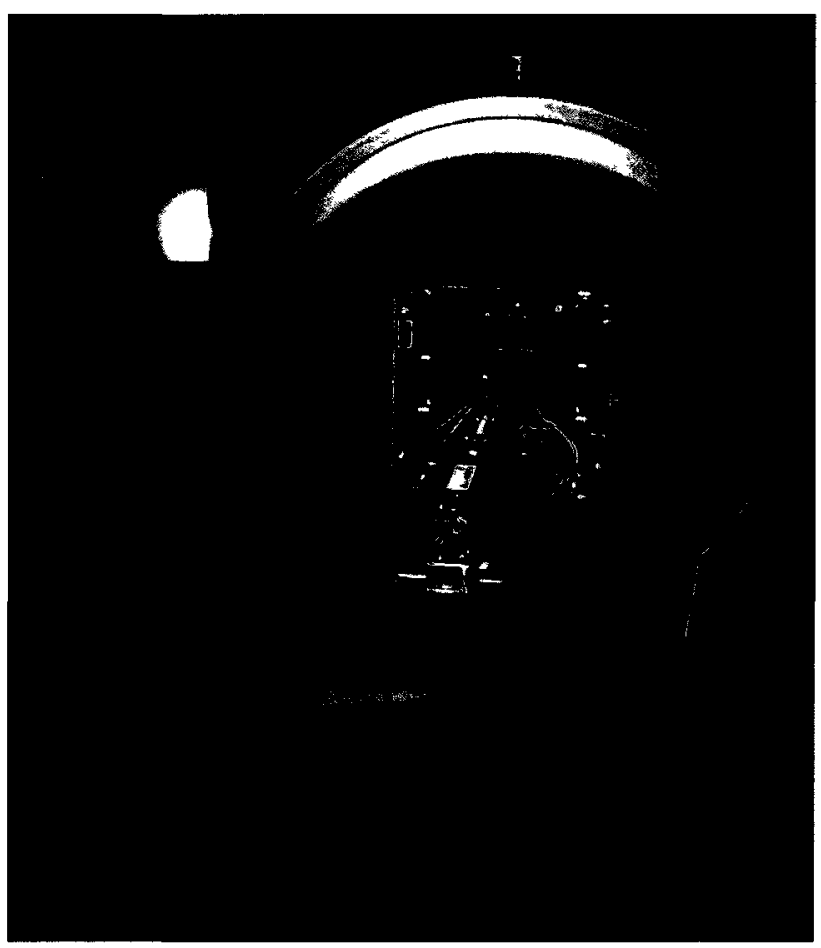

\section{Figure 2.2: MicroSPECT scanner at University of Ottawa Heart Institute}

All the animals were imaged under general anaesthesia of $1-2 \%$ Isoflurane that was administered via a nose cone. The anaesthetised rats were placed on the heated scanner bed in supine position and the bed was maintained at a constant temperature of $35^{\circ} \mathrm{C}$ throughout the scan. In this experiment we maintained the temperature of animal bed while scanning because small animals are subject to hypothermia while they are under anaesthesia. At this position the rats were connected with the ECG electrodes inserted subcutaneously. The ECG trace was measured using a Scientific Instruments monitor (SA Instruments Inc. Stony Brook, New York, USA. Model $1025 \mathrm{~T})$ and this monitor was used to gate the micro SPECT acquisitions.

All the 8 normal rats underwent gated SPECT scanning weekly for five weeks. The 8 MI (Myocardial Infarcted) rats underwent 5 gated SPECT acquisitions over 2 weeks with a period of 2-3 days between scanning. The scanning was 30 minutes in 
total duration acquired in three 10-minute segments and began immediately after injection of $0.5 \pm 0.05 \mathrm{mCi}$ of $\mathrm{Tl}-201$.

All the MI rats needed four weeks to recover from the surgery. Thus the MI scans began four weeks after the surgery. For MI rats only, a single 10-minute prescan was acquired prior to the injection. The pre-scan was subtracted from the other scans to correct for any residual activity from earlier imaging sessions due to the long physical half-life of Tl-201 ( $\mathrm{T}_{1 / 2}=3$ days $)$.

The total number of projections for both of the rats (MI and Normal) was 48 with 6 projections per head acquired over a camera rotation of 90 degrees. The total number of counts acquired was 3-4 million. Physiological parameters - the heart rate and the respiratory rate - were monitored for all of the scans. The acquisitions were ECG-gated into eight time frames per cardiac cycle. Table 2.1 shows the physiological data of our experiment. 


\begin{tabular}{|c|c|c|c|c|c|c|c|c|}
\hline Species & \multicolumn{2}{|c|}{ Number of Rat } & \multirow{2}{*}{$\begin{array}{r}\text { Scan } 1 \\
381.2\end{array}$} & \multirow{2}{*}{$\begin{array}{r}\text { Scan } 2 \\
358.7\end{array}$} & \multirow{2}{*}{$\begin{array}{r}\text { Scan } 3 \\
347.8\end{array}$} & \multirow{2}{*}{$\begin{array}{r}\text { Scan } 4 \\
362.4\end{array}$} & \multirow{2}{*}{$\begin{array}{r}\text { Scan } 5 \\
361.0\end{array}$} & \multirow[t]{2}{*}{ Scan 6} \\
\hline \multirow{16}{*}{$\begin{array}{c}\text { Normal } \\
\text { Rats }\end{array}$} & \multirow[t]{2}{*}{ Rat 1} & HR & & & & & & \\
\hline & & RR & 68.2 & 68.3 & 63.0 & 74.0 & 84.5 & \\
\hline & \multirow[t]{2}{*}{ Rat 2} & HR & 394.4 & 370.8 & 372.5 & 383.6 & 367.5 & \\
\hline & & RR & 77.6 & 64.0 & 73.3 & 59.6 & 71.0 & \\
\hline & \multirow[t]{2}{*}{ Rat 3} & HR & 399.9 & 376.8 & 380.3 & 382.2 & 361.2 & \\
\hline & & RR & 88.5 & 75.8 & 79.0 & 84.5 & 73.0 & \\
\hline & \multirow[t]{2}{*}{ Rat 4} & HR & 333.6 & 335.5 & 313.5 & 308.8 & 328.7 & 321.3 \\
\hline & & RR & 50.2 & 57.5 & 51.0 & 48.8 & 53.0 & \\
\hline & \multirow[t]{2}{*}{ Rat 5} & HR & 356.0 & 330.8 & 331.2 & 328.6 & 308.4 & \\
\hline & & $\mathrm{RR}$ & 52.0 & - & 50.0 & 48.4 & 42.5 & \\
\hline & \multirow[t]{2}{*}{ Rat 6} & HR & 338.3 & 302.6 & 323.8 & 294.0 & 291.3 & \\
\hline & & RR & 67.3 & 61.8 & 69.3 & 67.0 & 54.0 & \\
\hline & \multirow[t]{2}{*}{ Rat 7} & HR & 347.2 & 343.5 & 347.5 & 346.8 & 334.0 & 339.7 \\
\hline & & RR & 59.2 & 68.5 & 69.0 & 68.4 & 83.2 & 53.7 \\
\hline & \multirow[t]{2}{*}{ Rat 8} & HR & 354.0 & 365.5 & 357.3 & 385.4 & 356.0 & 371.0 \\
\hline & & RR & 57.3 & 62.3 & 58.3 & 69.0 & 44.0 & 69.7 \\
\hline \multirow{16}{*}{$\begin{array}{c}\text { MI } \\
\text { Rats }\end{array}$} & \multirow[t]{2}{*}{ Rat 1} & HR & 339.6 & 359.8 & 351.2 & 364.4 & 376.7 & \\
\hline & & RR & 61.8 & 51.4 & 60.2 & 51.1 & 59.2 & \\
\hline & \multirow[t]{2}{*}{ Rat 2} & HR & 359.5 & 338.8 & 343.8 & 443.3 & 344.6 & \\
\hline & & RR & 52.8 & 42.8 & 48.5 & 50.5 & 50.4 & \\
\hline & \multirow{2}{*}{ Rat 3} & HR & 335.8 & 350.7 & 324.7 & 317.8 & 340.4 & \\
\hline & & RR & 49.8 & 61.7 & 51.0 & 52.3 & 56.0 & \\
\hline & \multirow[t]{2}{*}{ Rat 4} & HR & 342.6 & 312.5 & 313.8 & 295.5 & 293.8 & \\
\hline & & RR & 66.0 & 44.5 & 43.5 & 41.0 & 41.8 & \\
\hline & \multirow[t]{2}{*}{ Rat 5} & HR & 338.3 & 299.0 & 284.0 & 286.0 & 288.7 & \\
\hline & & RR & 61.8 & 46.0 & 47.6 & 52.0 & 53.7 & \\
\hline & \multirow[t]{2}{*}{ Rat 6} & HR & 292.5 & 275.8 & 276.5 & 264.3 & 271.3 & \\
\hline & & RR & 50.0 & 56.3 & 52.3 & 34.7 & 50.8 & \\
\hline & \multirow[t]{2}{*}{ Rat 7} & HR & 353.0 & 328.7 & 321.5 & 307.3 & 349.3 & \\
\hline & & RR & 61.8 & 62.0 & 64.0 & 65.3 & 51.8 & \\
\hline & \multirow[t]{2}{*}{ Rat 8} & HR & 378.0 & 352.6 & 345.2 & 339.0 & 375.8 & \\
\hline & & $\mathrm{RR}$ & 61.0 & 63.2 & 61.3 & 65.4 & 58.3 & \\
\hline
\end{tabular}




\subsubsection{Reconstruction}

To reconstruct the acquired data of the pinhole gated SPECT studies we used the OS-EM iterative reconstruction algorithm (Hudson HM 1994). The implementation of the reconstruction algorithm was with vendor-supplied software that included collimator modelling but no attenuation correction (Di Filippo, FP 2008; Forrer F 2006). Three iterations in combination with eight subsets were used for a total iteration number of twenty-four. The images were filtered post-reconstruction with a " $45 \%$ smoothing" filter, in this case corresponding to a Gaussian filter with a full-width at half-maximum (FWHM) of $0.63 \mathrm{~mm}$. We used the reconstruction parameter " $100 \%$ resolution" which resulted in a reconstructed voxel size of $0.6 \mathrm{~mm} \mathrm{x}$ $0.6 \mathrm{~mm}$ pixel size and $0.6 \mathrm{~mm}$ slice thickness. The average time for each gated reconstruction was 50 minutes.

\subsubsection{Weight Correction}

All of the 8 normal rats underwent scanning for 5 weeks and the MI rats for 2 weeks. As they were well fed and well treated in a good environment, they grew over the time of the study. The average weight of the normal rats was $245 \mathrm{~g}$ at day 1 and $410 \mathrm{~g}$ at day 28 (Figure 2.5). Similarly, the average weight of the MI rats was $446 \mathrm{~g}$ at day 1 whereas at day 14 it was $498 \mathrm{~g}$ (Figure 2.18). It is clear that, with the increasing weight of the body, the rats' hearts grow bigger over time. So, to correct for this known systematic error, we corrected the volumes (end diastolic volume (EDV) and end systolic volume (ESV)) for rats' weight gain. The correction was done using equation (2.1) which was based on measured ungated heart volumes from microSPECT scans with Tc-99m-tetrofosmin (Strydhorst, J 2010),

$$
C V o l=\operatorname{Vol}\left[1-\left(\frac{m_{i}}{M_{i}}-1\right) K\right]
$$


where $C V o l=$ corrected volume, $V o l=$ uncorrected volume, $m_{i}=$ weight of a rat for a single scan, $M_{i}=$ average weight of the same rat for 5 scans, $i=$ number of rats and $K$ $=0.76$, derived from ungated Tc-99m-tetrofosmin rat data.

\subsubsection{Data Analysis}

All the gated studies were imported into 4DM-SPECT for analysis. 4DMSPECT is a clinical software package from Invia Medical solutions, Ann-Arbor, MI. Using 4DM-SPECT, we measured the end diastolic volume, end systolic volume, ejection fraction and the tracer uptake in the myocardium normalized to the location of maximum uptake. Perfusion homogeneity was calculated as the standard deviation of the normalized myocardial uptake in the 17 segments of the heart (Cerqueira MD 2002).

After importing the data into 4DM-SPECT, the heart is aligned semiautomatically. The software attempts to identify the horizontal long axis, vertical long axis, and short axis planes which are then corrected manually. The operator then also assigns the position of the valve plane and the location of the apex. Following alignment, the software automatically calculated the EDV, ESV, EF and normalized 17-segment tracer uptake. The data were then exported to a text file and the homogeneity $(\mathrm{PH})$ was calculated manually. Figure 2.3 shows an example of the cardiac reorientation of left ventricular long axis in 4DM-SPECT. 


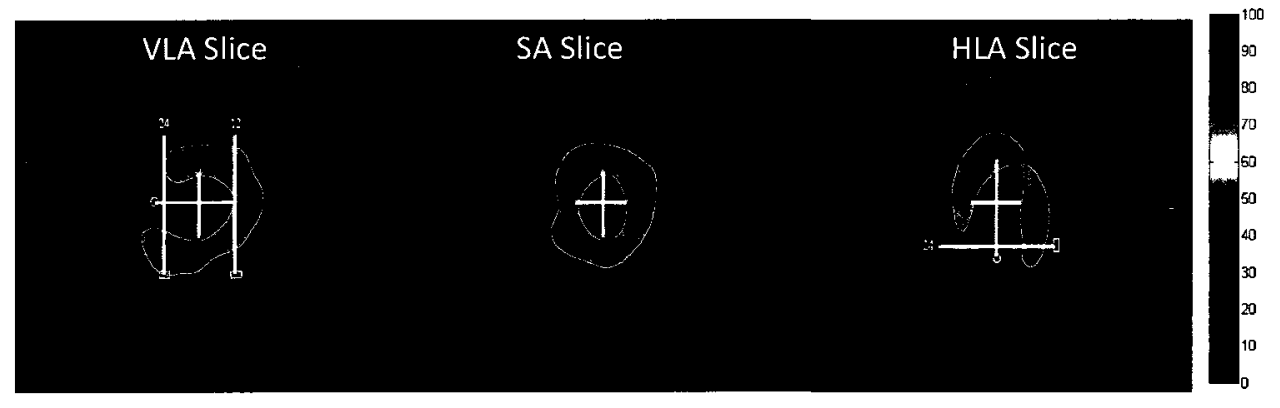

Figure 2.3: Cardiac reorientation of left ventricular long axis slices. The uptake in the rat heart is shown in a pseudo-color scale as three orthogonal views. The white indicator lines are manipulated by the user to rotate the heart into the correct orientation and define the length of the heart along the long axis. 


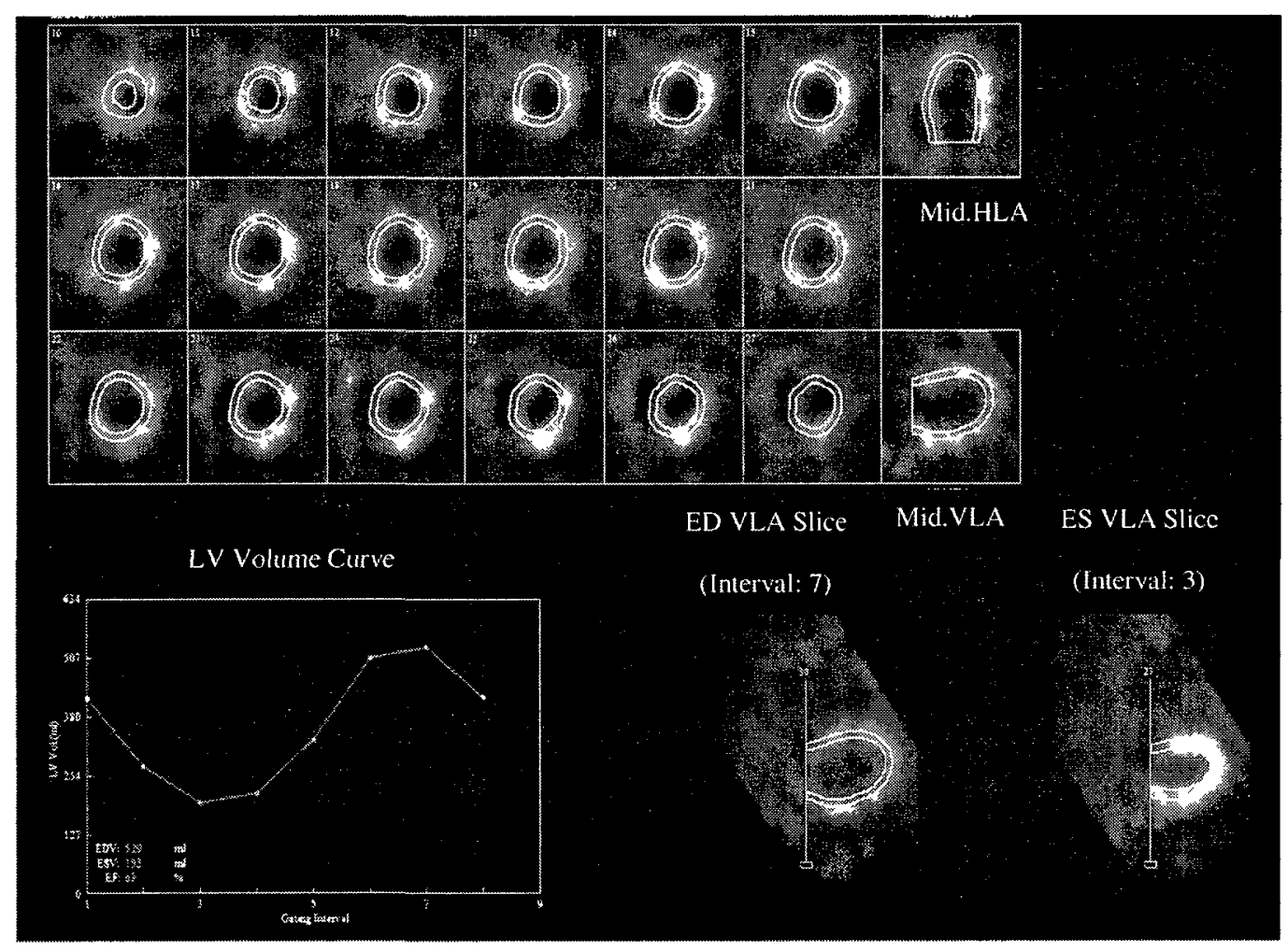

Figure 2.4: Example of an image of a normal rat displayed in the 4DMSPECT software package. Contours indicate where the software has identified the heart wall over the slices of the heart. The curve at the bottom left shows its calculation of the ventricular volume and ejection fraction over the cardiac cycle. The figures in the lower right are used to define the valve plane at the base of the ventricle during diastole and systole.

Figure 2.4 shows an example of pinhole gated myocardial perfusion SPECT study for a normal rat in the 4DM-SPECT. The figure represents vertical long axis (VLA), short axis (SA), and horizontal long axis (HLA) slices, of the rat heart.

The intra- and inter-subject reproducibility for 8 normal and $8 \mathrm{MI}$ rats were calculated separately with MS-Excel. We considered all 40 scans acquired: the 8 
normal/MI rats each with 5 different scans. For intra-subject variability, the standard deviation of the 5 different scans was calculated for each of the rats and then averaged over the 8 different rats. The error was the standard error of 8 different measurements. In a similar way, for inter-subject variability of each of the parameters, we took the standard deviation of the 8 different rats for a single scan and then averaged over the 5 different scans. The error on that was the standard error of 5 different scans. We analyzed all of our results in a similar fashion for each parameter: EDV, ESV, and EF.

The perfusion homogeneity for each of the rats and each of the scans was measured in a different way than EDV, ESV and EF. We used a 17 segment model for measuring perfusion homogeneity. For each of the rats, for each of the scans we have 17 different numbers which are the average uptake per segment. We took the standard deviation of these 17 numbers which was the perfusion homogeneity for a single rat for that single scan. The intra- and inter-subject variability in perfusion homogeneity were then calculated in the same fashion as described for the EDV, ESV, and EF.

Finally, the variability in segmental perfusion was assessed. As there are 17 segments for each scan, the variability was calculated separately for each segment in the same fashion as the EF and then only the average over the 17 segments is reported.

Normal databases are frequently used in clinical SPECT to assist in the evaluation of size and severity of myocardial perfusion defects. To allow this approach with our rat data, we made a normal database of our data. A normal data base was constructed for each different time duration. The databases were constructed with all of the 40 scans ( 8 rats $\times 5$ scans). Then we took the average and 
standard deviation of all these 40 scans for each of the 17 segments and used these to get the polar map.

We also calculate the z-score of our measurement of tracer uptake for the MI rats. In general, a z-value is the expression of the divergence of a specific result (experimental result) from the most desirable result (mean) as a number of standard deviation. The general formula for $z$-value is

$$
Z=\frac{x-\bar{x}}{\mu}
$$

where $x$ is the experimental result, $\bar{x}$ is the mean and $\mu$ is the standard deviation.

The $\mathrm{z}$-value for each of the segments was calculated using equation 2.2 and the final $z$-score was the sum of all the z-values which are smaller than -2 . Our z-score represents the divergence of the segmental perfusion of MI rats from the normal database and indicates the size and severity of the infarct.

We plotted all of our graphs with the absolute values of the parameter (EDV, $\mathrm{ESV}, \mathrm{EF}$ and $\mathrm{PH}$ ) and our results are the standard deviation of these values.

The statistical method ANOVA (ANalysis Of VAriance) was applied for testing the hypothesis. Specifically, the single factor ANOVA was used in all the cases. P-values $\leq 0.05$ were considered significant.

The student T-test was used to test for significant differences in the intra- and inter-rat uncertainties measured and for differences between the normal and MI rat uncertainties. Two samples assuming unequal variances t-test was used for all of the 10 minute, 20 minute and 30 minute cases. (Table. 2.2) The reproducibility of EDV, ESV, EF, and PH were all tested. T-test is basically used to find whether the means 
of two groups are statistically same or different than each other. In this case, the set of values being compared are the uncertainties measured and the 'unequal variances' are the uncertainties in those uncertainties. The $p<0.05$ was considered significantly different.

\subsection{Results}

\subsubsection{Normal Rats}

\subsubsection{10 Minute Study}

The body weight of 8 normal rats is shown in figure 2.5 . The average weight of the 8 normal rats was $246 \pm 7 \mathrm{~g}$ on day 1 and $400 \pm 16 \mathrm{~g}$ on day 28 . The mean weight was $342 \pm 59 \mathrm{~g}$ for 8 normal rats.

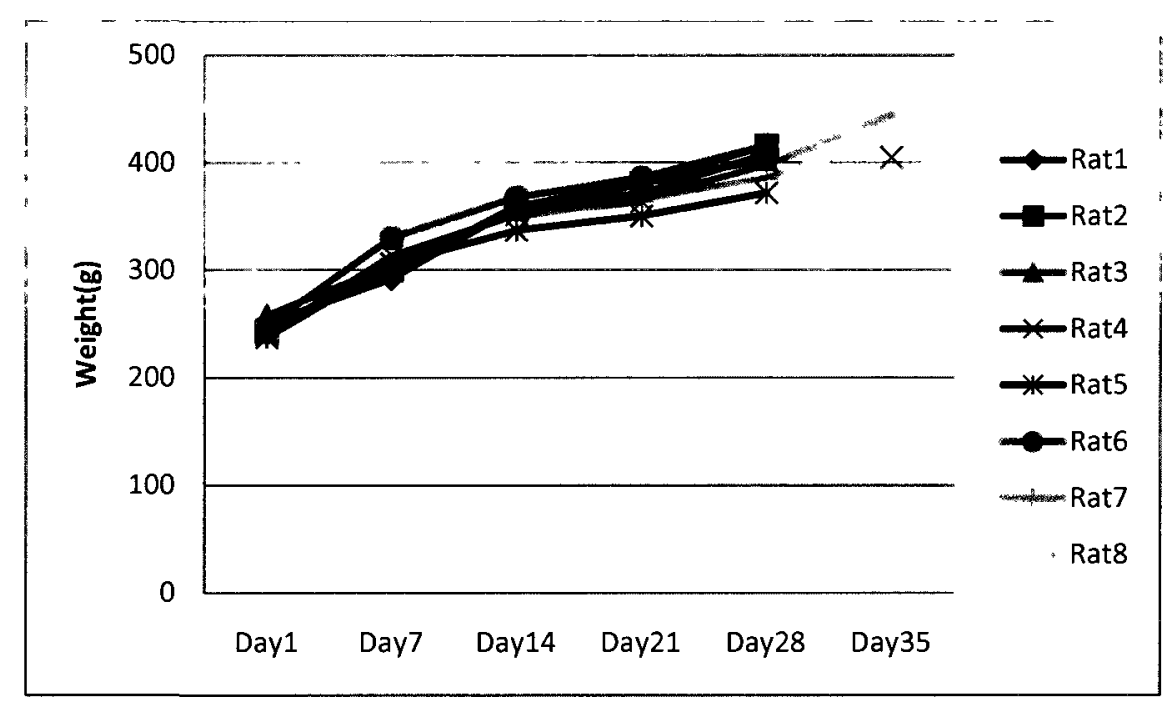

Figure 2.5: Weights of the 8 normal rats during the study.

For three of the rats, we were unable to obtain images every week and we had to do a final scan on day 35 . We faced a camera problem for two of the rats. The camera was then fixed and did a final scan at the end of the study to make up for the 
lost data. One of the rats woke up during scanning, so that scan was removed from the study and we replaced it with a scan on the $6^{\text {th }}$ week.

Figure 2.6 shows the variability of left ventricular uncorrected volumes: end diastolic volume and end systolic volume. The average standard deviation of end diastolic volume (uncorrected) calculated from the measurement was $73 \pm 4 \mathrm{~mm}^{3}$ across scans and $40 \pm 4 \mathrm{~mm}^{3}$ across rats. The average standard deviation of end systolic volume (uncorrected) across scans was $34 \pm 3 \mathrm{~mm}^{3}$ and across rats it was $27 \pm$ $6 \mathrm{~mm}^{3}$. 
(a)

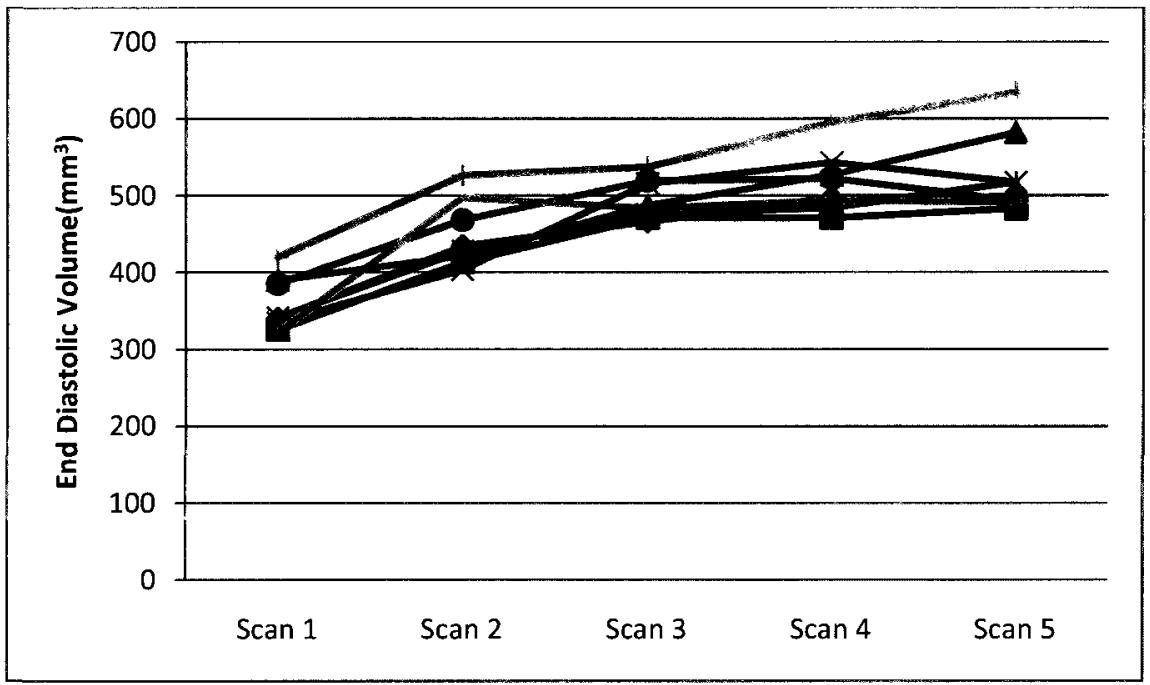

(b)

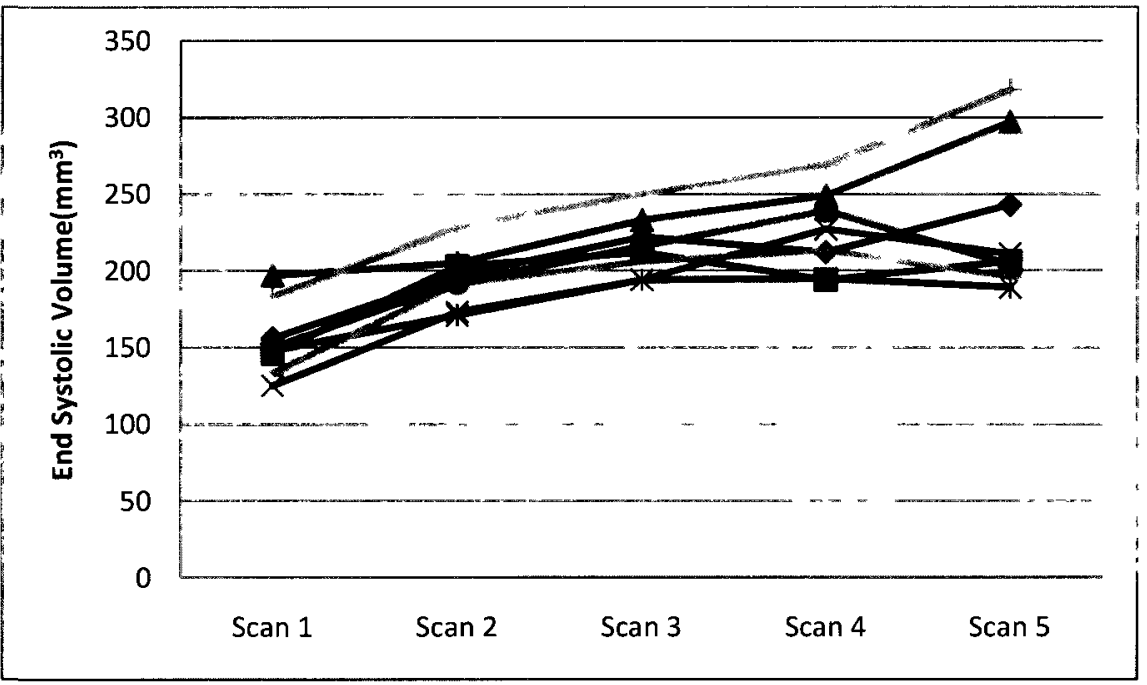

Figure 2.6: (a) End diastolic volume (EDV) and (b) end systolic volume (ESV) for 8 normal rats (uncorrected) and 5 different scans using a 10 minute scan duration. Legend is as in Figure 2.5.

As we mentioned before, the weight of the rats increased during the study so we corrected the heart volumes for the rats' weight gain. Figure 2.7 shows the reproducibility of the corrected volumes. The standard deviation of the corrected end diastolic volume was $30 \pm 3 \mathrm{~mm}^{3}$ across scans and $45 \pm 5 \mathrm{~mm}^{3}$ across rats. The 
standard deviation of corrected end systolic volume was $18 \pm 2 \mathrm{~mm}^{3}$ across scans and $29 \pm 5 \mathrm{~mm}^{3}$ across rats.

(a)
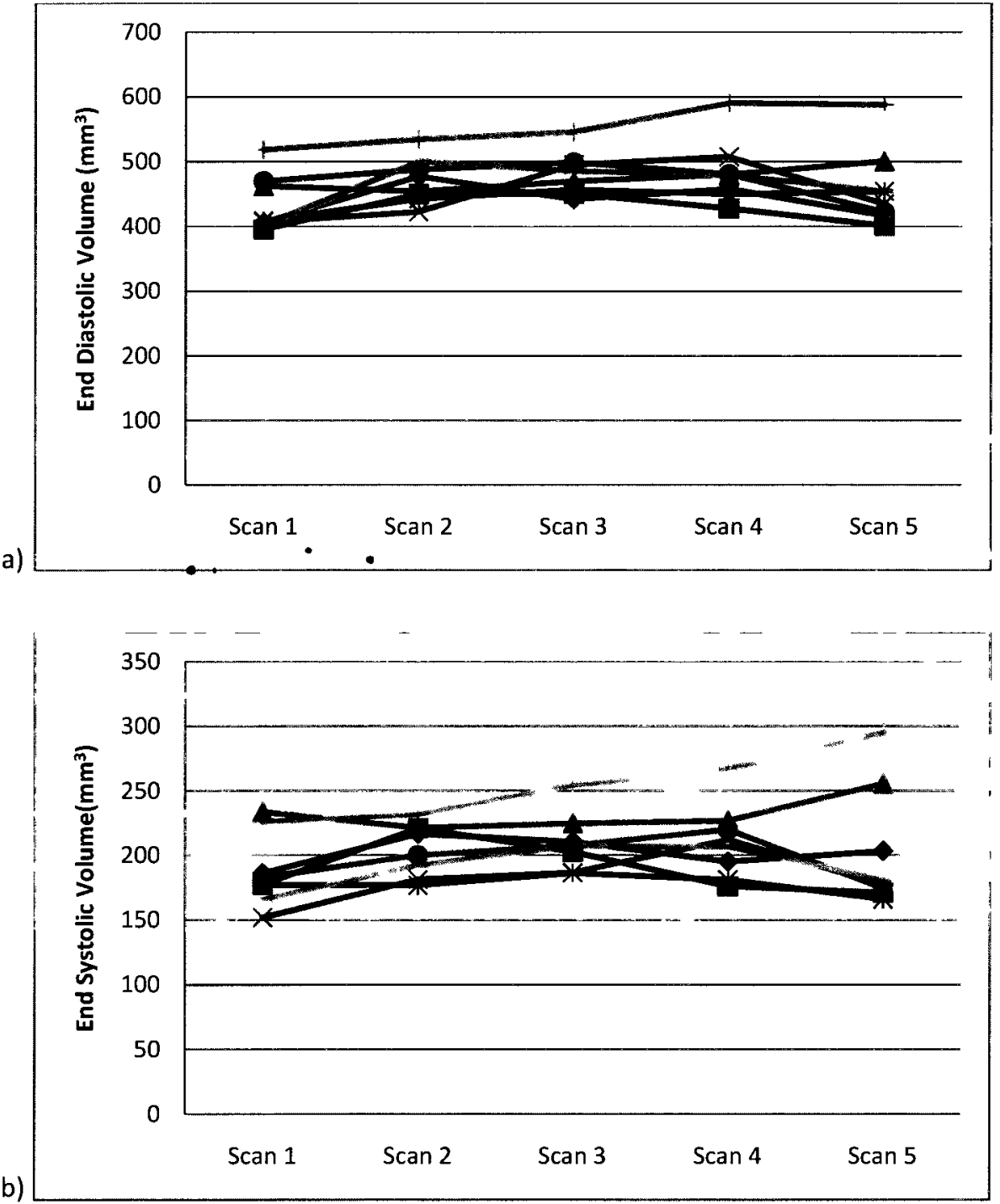

Figure 2.7: Weight-corrected volumes (a) EDV and (b) ESV of 10 minute scan duration for the $\mathbf{8}$ normal rats. Legend is as in Figure

\section{5 .}

The ejection fraction and perfusion homogeneity for each rat and each scan are shown in figure 2.8. The standard deviation of ejection fraction across scans was $2 \pm$ 
$0.2 \%$ and across rats was $4 \pm 1 \%$. The intra- and inter-subject reproducibility of perfusion homogeneity was $7 \pm 0.1 \%$ and $7 \pm 0.1 \%$, respectively.

(a)

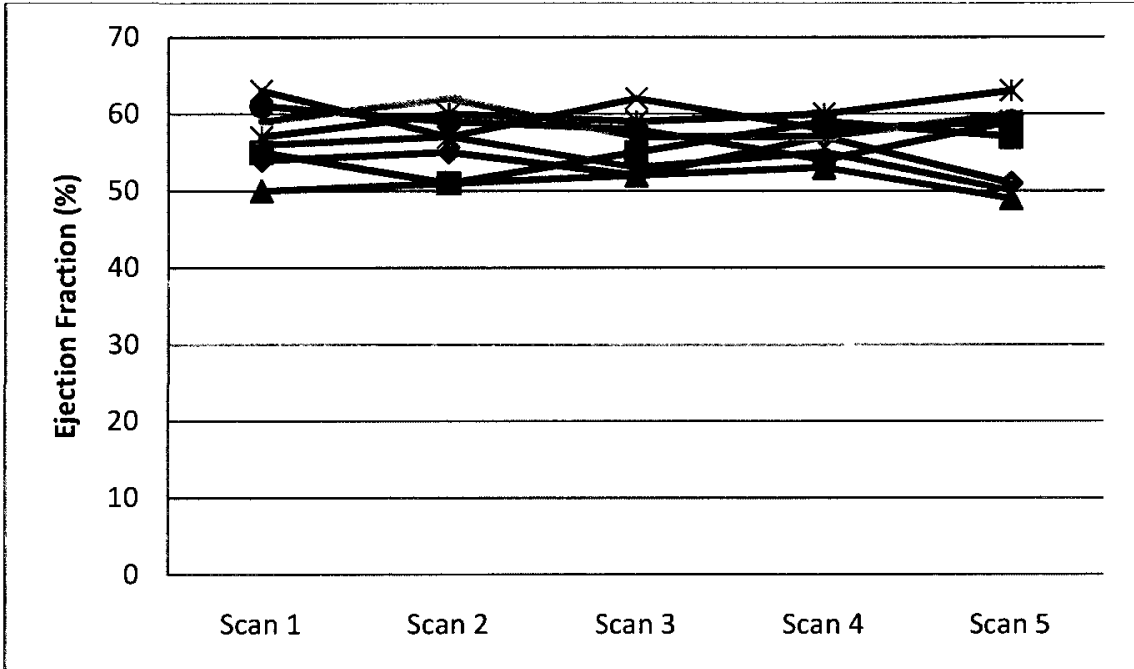

(b)

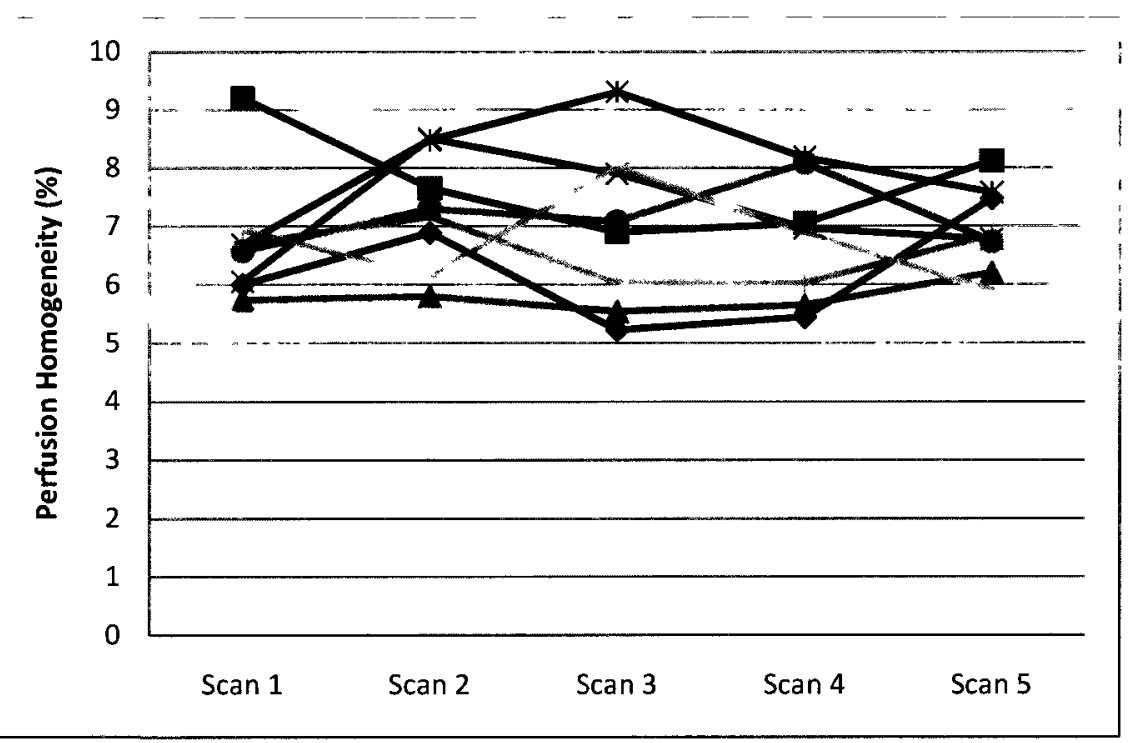

Figure 2.8: (a) Ejection fraction and (b) perfusion homogeneity of 8 normal rats for a 10 minute scan duration. Legend is as in Figure

\section{5.}

A polar map of the 17-segment normal uptake database for 10 minute scan duration is shown in the figure 2.9 where the standard deviation of uptake in each 
segment is indicated by the number and the mean value of uptake is indicated by the colour. The normal database is constructed from all 40 of the normal (ungated) scans.

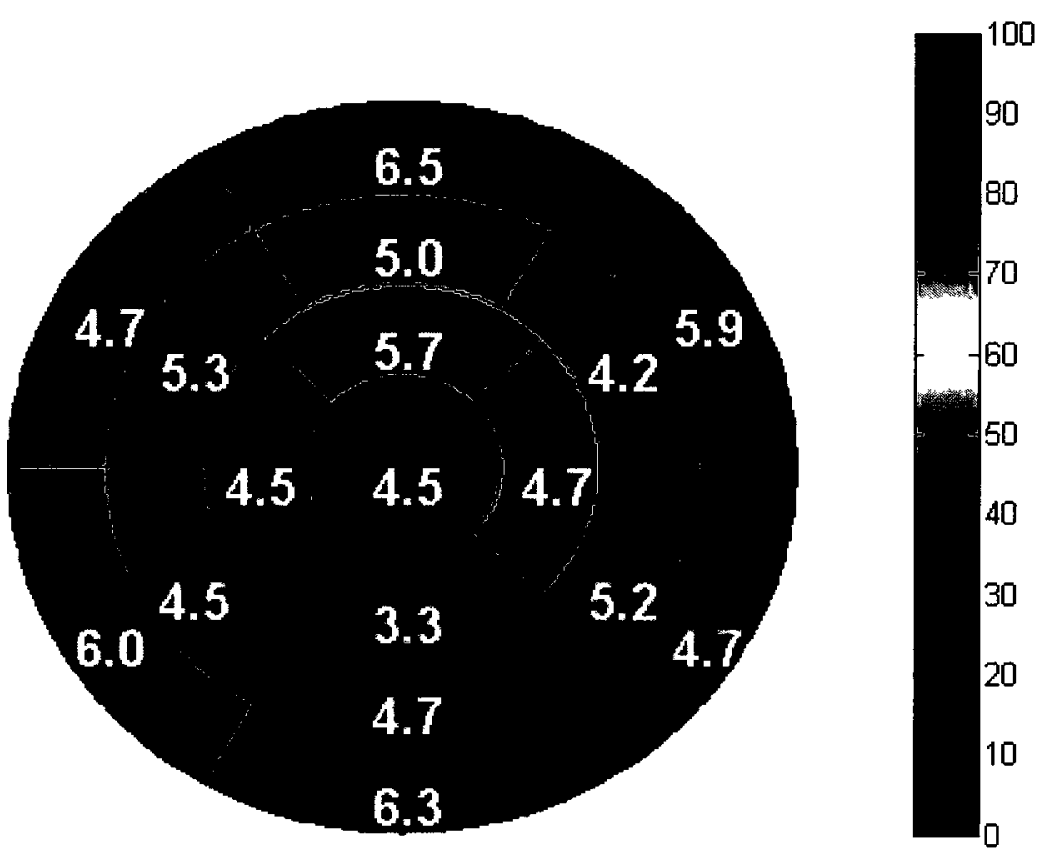

Figure 2.9: Polar map of normal database for 10 minute scan duration. Mean values of the uptake of tracer in each segment are indicated by the colour bar. The standard deviation in the uptake in each segment is indicated by the number overlain on that segment.

\subsubsection{20 Minute Study}

In the 20 minute study, the intra- and inter-subject reproducibility of uncorrected end diastolic volumes were found to be $68 \pm 4 \mathrm{~mm}^{3}$ and $35 \pm 6 \mathrm{~mm}^{3}$ and 
for systolic volume they were $33 \pm 2 \mathrm{~mm}^{3}$ and $22 \pm 4 \mathrm{~mm}^{3}$ respectively. Figure 2.10 shows the reproducibility of uncorrected EDV and ESV.

(a)

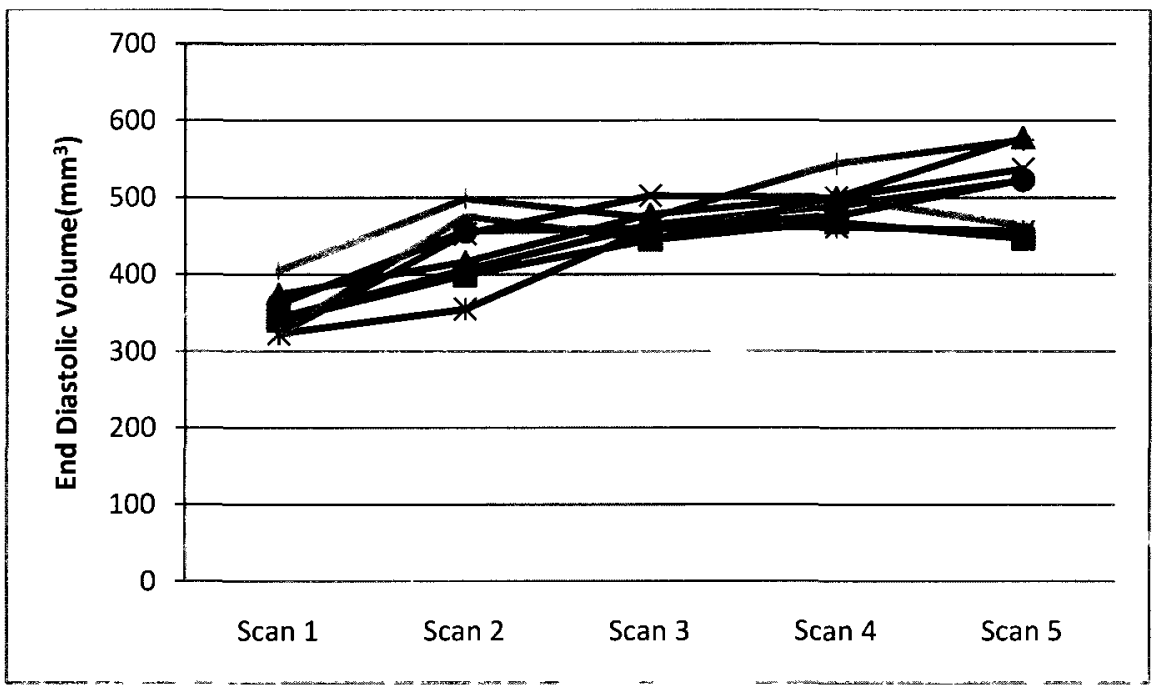

(b)

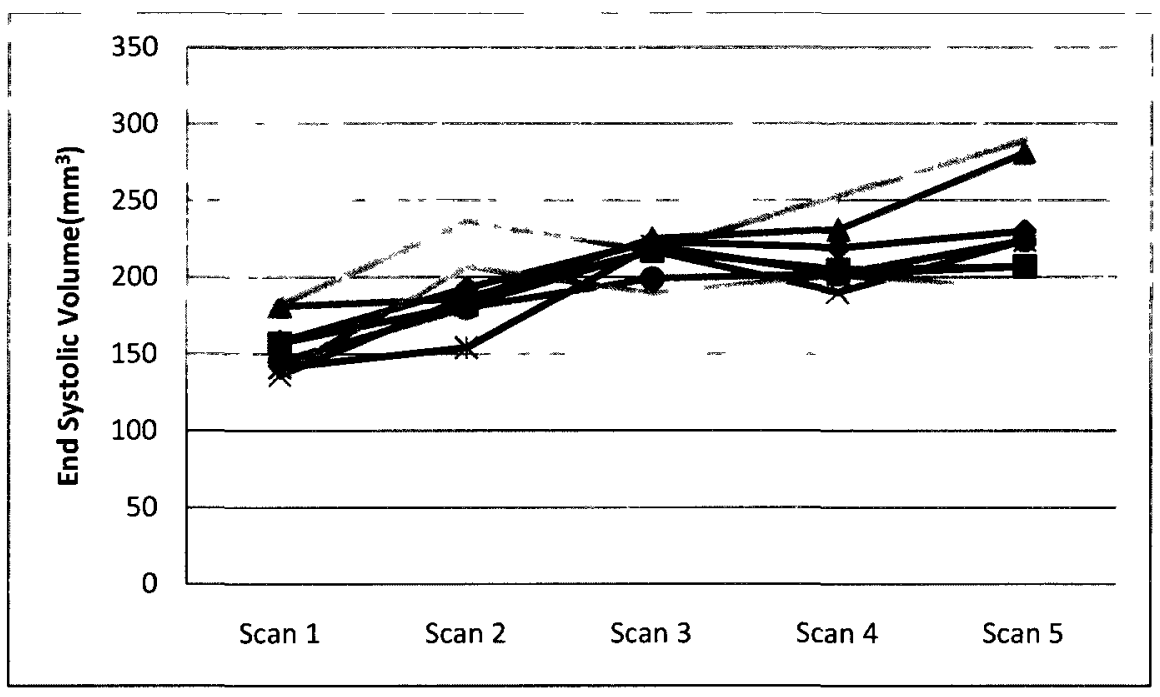

Figure 2.10: Reproducibility of uncorrected (a) EDV and (b) ESV

for inter- and intra-subject of 8 normal rats for 20 minute scan. Legend is as in Figure 2.5.

The result of the investigation of the intra-subject reproducibility of EDV (corrected) and ESV (corrected) are $25 \pm 3 \mathrm{~mm}^{3}$ and $15 \pm 2 \mathrm{~mm}^{3}$ respectively. 
Alternatively, the inter-subject reproducibility of EDV and ESV are $38 \pm 5 \mathrm{~mm}^{3}$ and $23 \pm 4 \mathrm{~mm}^{3}$, respectively. Figure 2.11 illustrates the inter- and intra-subject reproducibility of weight corrected EDV and ESV.

(a) i.
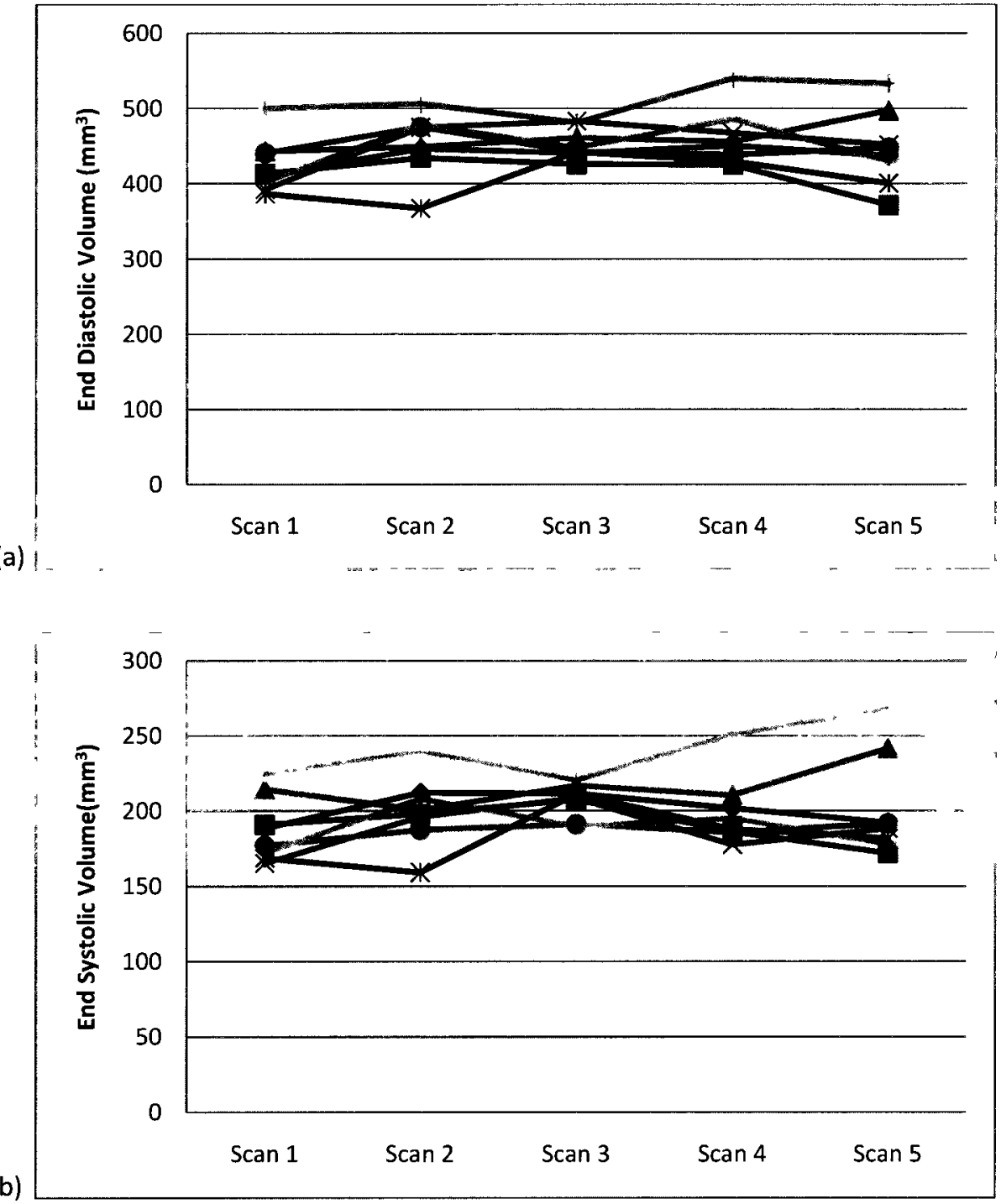

Figure 2.11: Reproducibility of weight corrected (a) EDV and (b)

ESV for 20 minute scan duration of 8 normal rats. Legend is as in Figure

2.5. 
Figure 2.12 shows the reproducibility of ejection fraction and perfusion homogeneity for 20 minute study. The average of standard deviation of ejection fraction is $2 \pm 0.1 \%$, across scans and $3 \pm 0.1 \%$ across rats. The average of perfusion homogeneity across scans is $7 \pm 0.1 \%$ and $7 \pm 0.2 \%$ across rats.

(a)

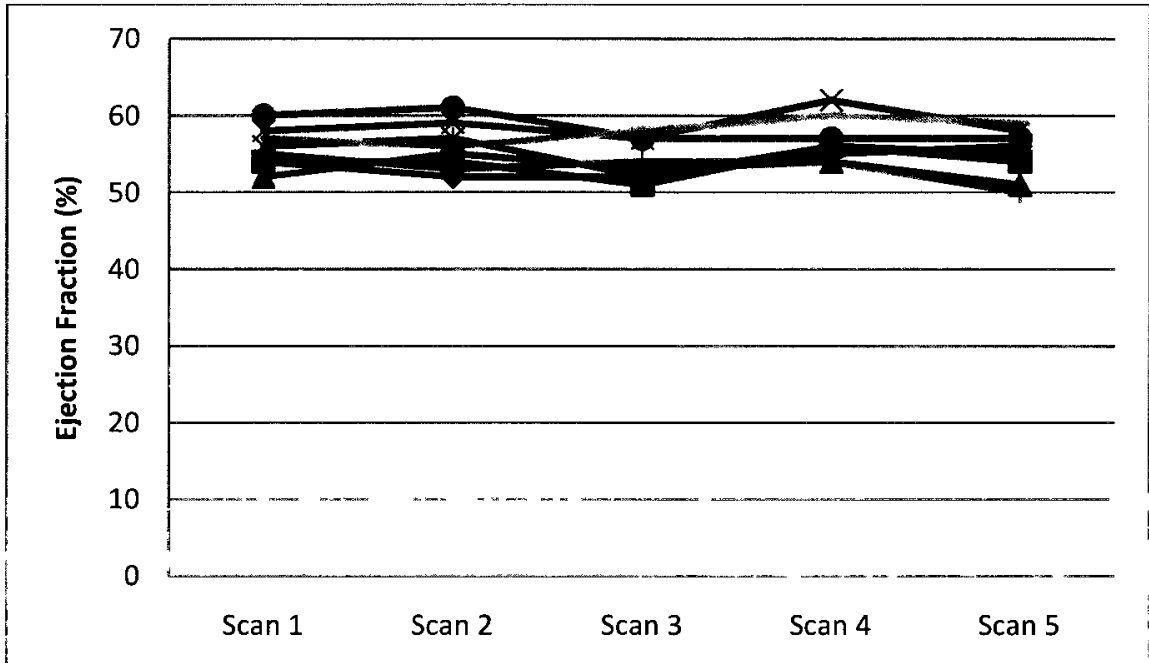

(b)

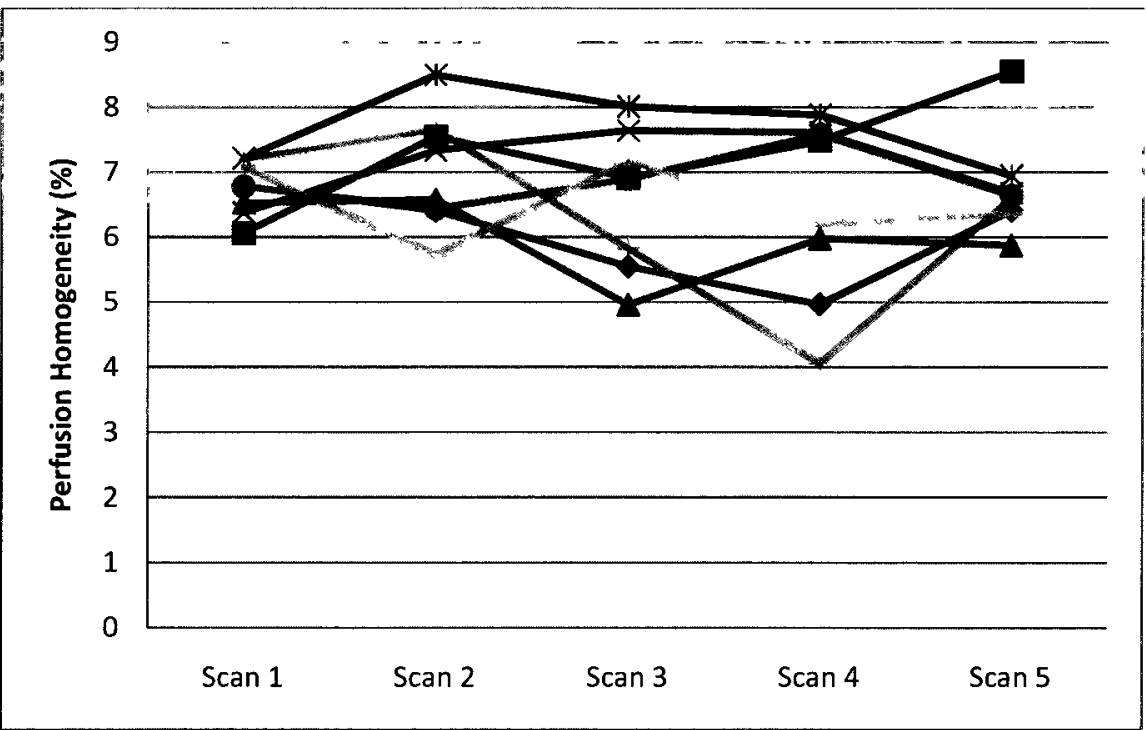

Figure 2.12: Inter- and intra-subject reproducibility of (a) EF and

(b) PH for a 20 minute scan duration of 8 normal rats. Legend is as in

Figure 2.5. 
A polar map of the normal database is shown in the figure 2.13 for 20 minute study.

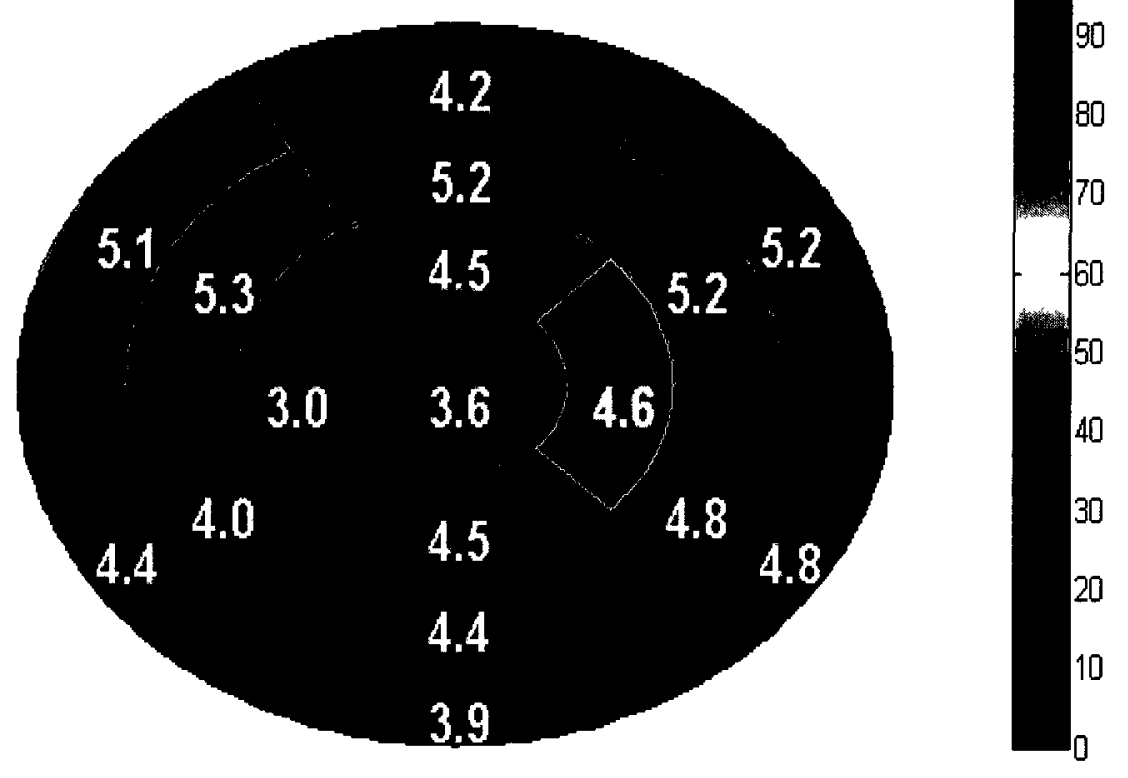

Figure 2.13: Polar map of normal database for 20 minute study. Mean values of the uptake of tracer in each segment are indicated by the colourbar. The standard deviation in the uptake in each segment is indicated by the number overlain on that segment.

\subsubsection{30 Minute Study}

The result of the assessment, for 30 minute study, of intra- and inter-subject reproducibility in the pinhole gated myocardial perfusion SPECT are demonstrated in the figures in this section. No significant differences were found for left ventricular volumes in the inter- and intra-subject cases. Intra-subject reproducibility for uncorrected EDV and ESV are $71 \pm 3 \mathrm{~mm}^{3}$ and $33 \pm 1 \mathrm{~mm}^{3}$ respectively whereas the inter-subject reproducibility for those parameters are $33 \pm 3 \mathrm{~mm}^{3}$ and $26 \pm 3 \mathrm{~mm}^{3}$ 
respectively. For corrected rat weight gain, the intra-subject variability of EDV and ESV are $29 \pm 4 \mathrm{~mm}^{3}$ and $15 \pm 2 \mathrm{~mm}^{3}$ and the inter-subject reproducibility are $38 \pm 3$ $\mathrm{mm}^{3}$ and $28 \pm 2 \mathrm{~mm}^{3}$, respectively. Figure 2.14 shows the inter- and intra-subject reproducibility for uncorrected left ventricular volumes and figure 2.15 shows the inter- and intra-subject reproducibility for corrected volumes.

(a)

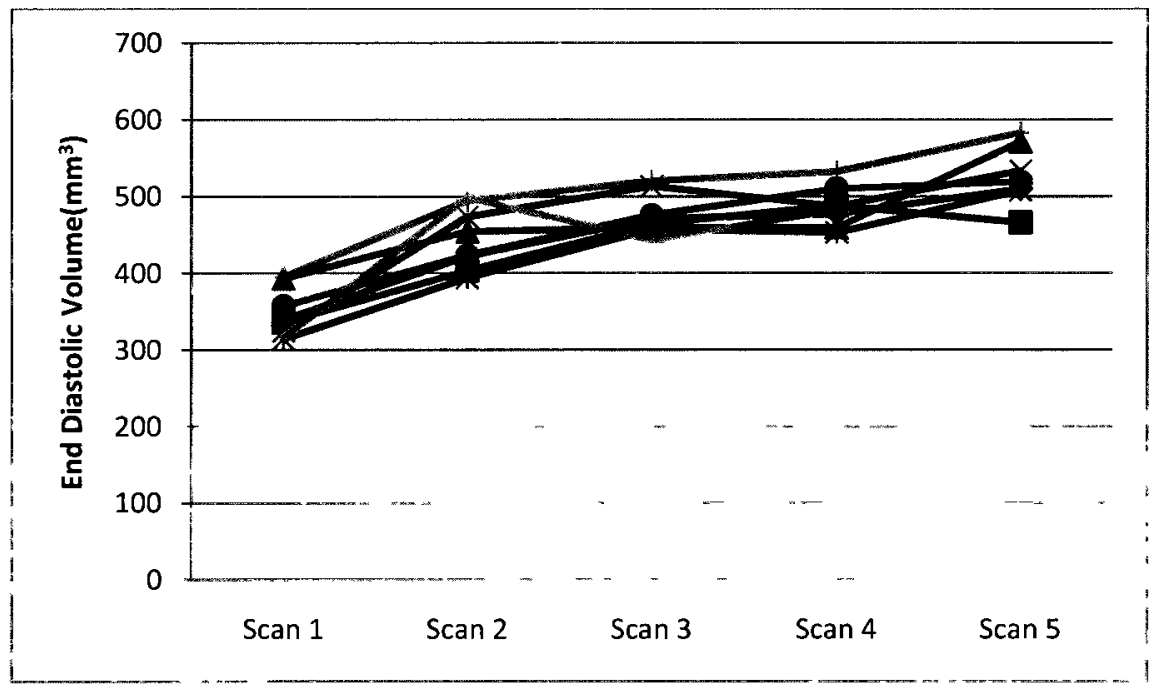

(b)

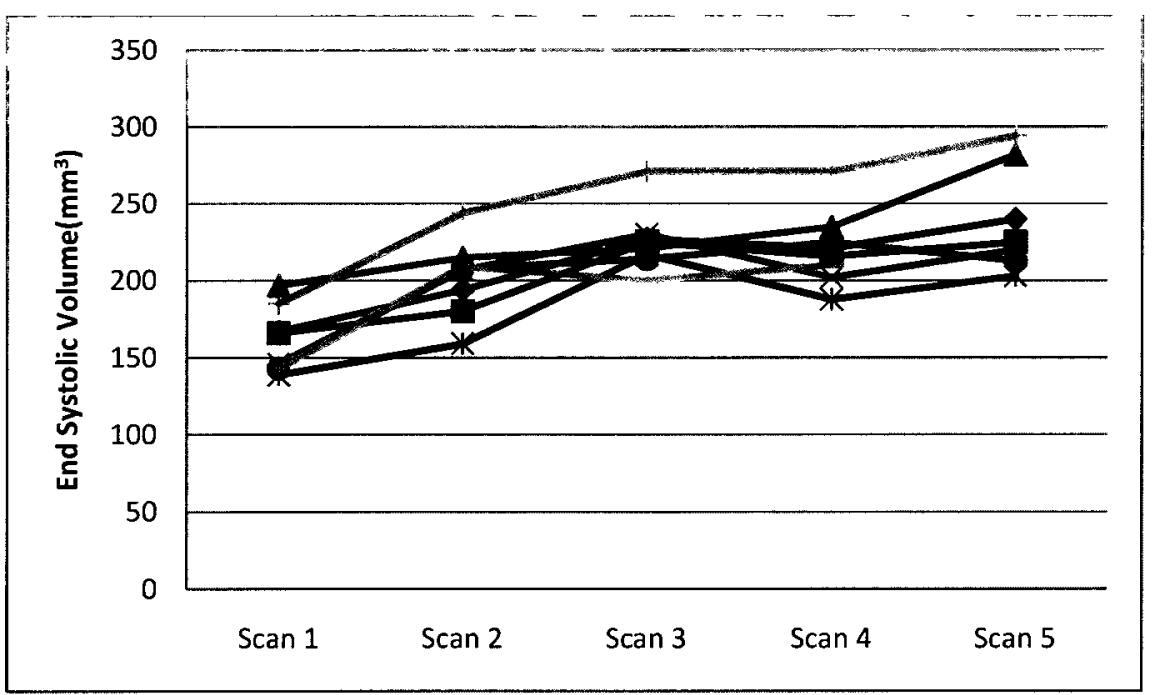

Figure 2.14: Inter- and intra-subject reproducibility of uncorrected (a) end diastolic volume (EDV) and (b) end systolic volume (ESV) of 30 minute scan of 8 normal rats. Legend is as in Figure 2.5 . 
(a)

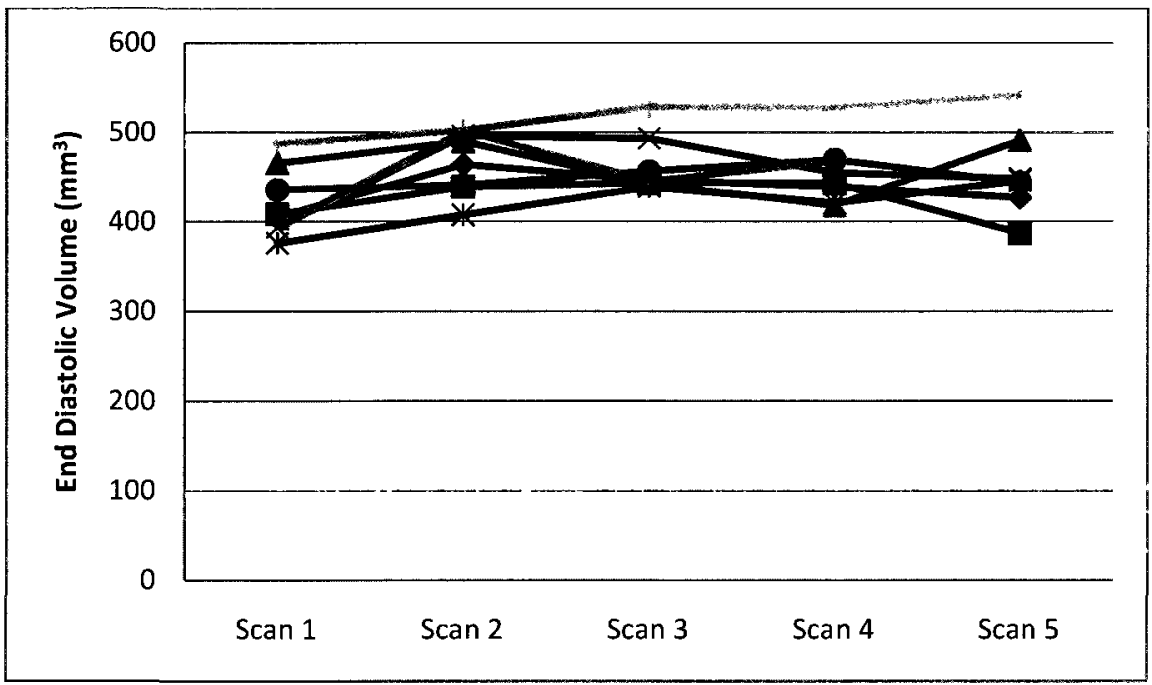

(b)

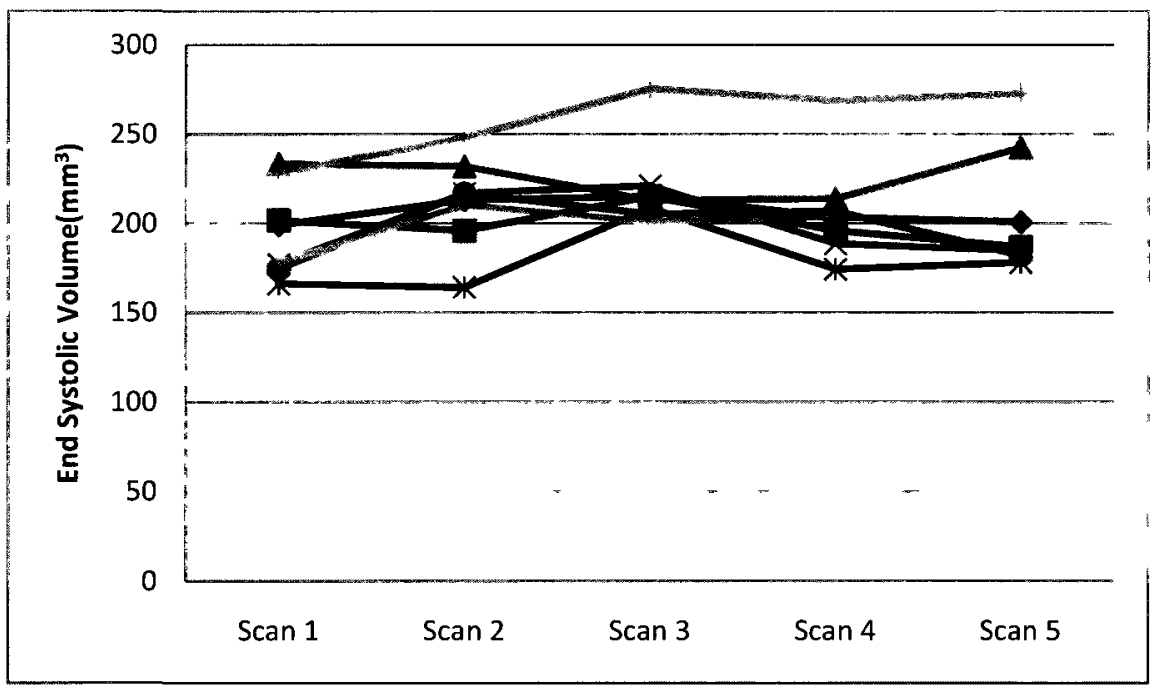

Figure 2.15: Inter- and intra-subject reproducibility for (a) EDV (corrected) and (b) ESV (corrected) for the 30 minute study of 8 normal rats. Legend is as in Figure 2.5 .

The results of measurement for the reproducibility of ejection fraction and perfusion homogeneity are shown in figure 2.16. No significant differences were found in these cases also. The intra-subject reproducibility for ejection fraction and perfusion homogeneity are $2 \pm 0.3 \%$ and $7 \pm 0.2 \%$, respectively. On the other side, the inter-subject reproducibility of ejection fraction is $3 \pm 0.3 \%$ and of perfusion 
homogeneity, $7 \pm 0.3 \%$. The reproducibility of perfusion homogeneity are exactly the same for inter- and intra-subject study.

(a)

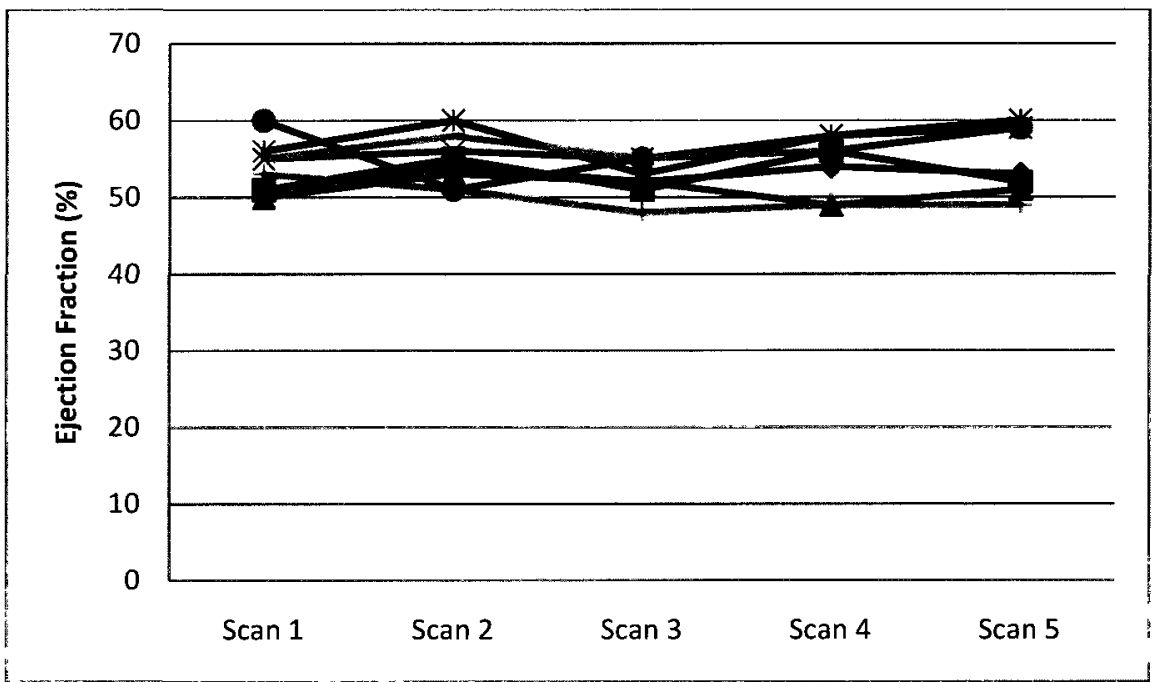

(b)

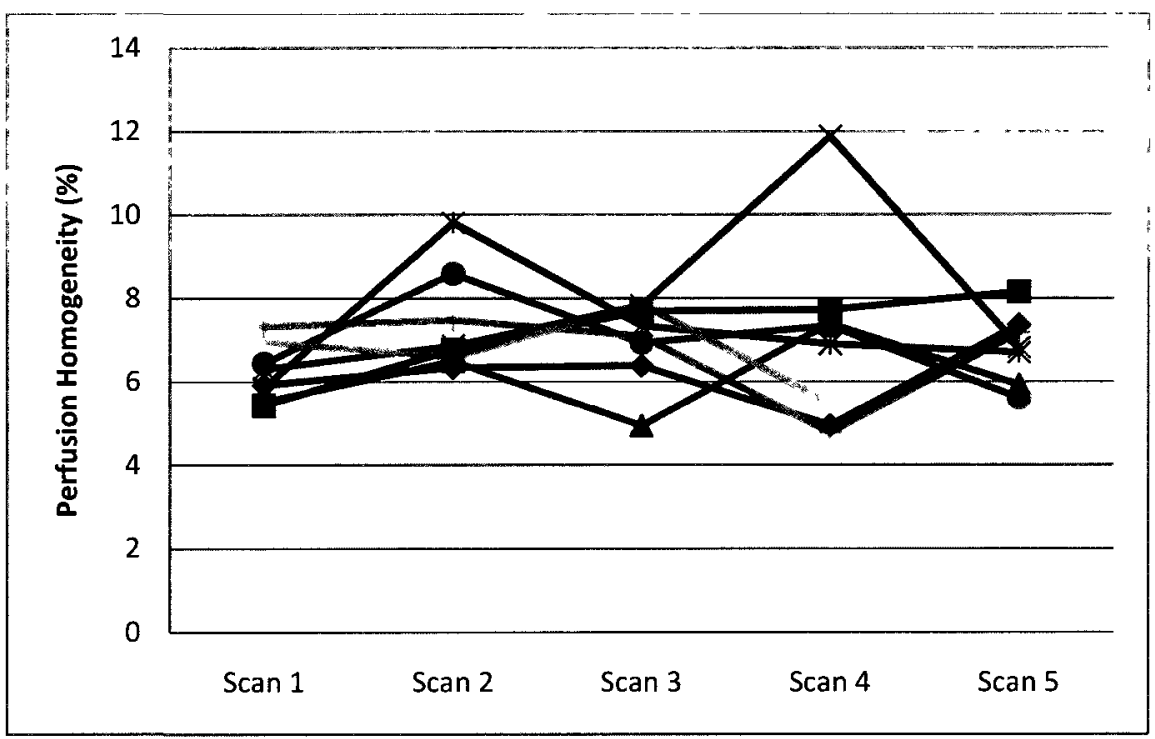

Figure 2.16: Variability of (a) ejection fraction (EF) and (b)

perfusion homogeneity (PH) for 30 minute scan duration of 8 normal rats.

Legend is as in Figure 2.5. 
For the normal database of 30 minute scan, the polar map of myocardial uptake is shown in figure 2.17 .

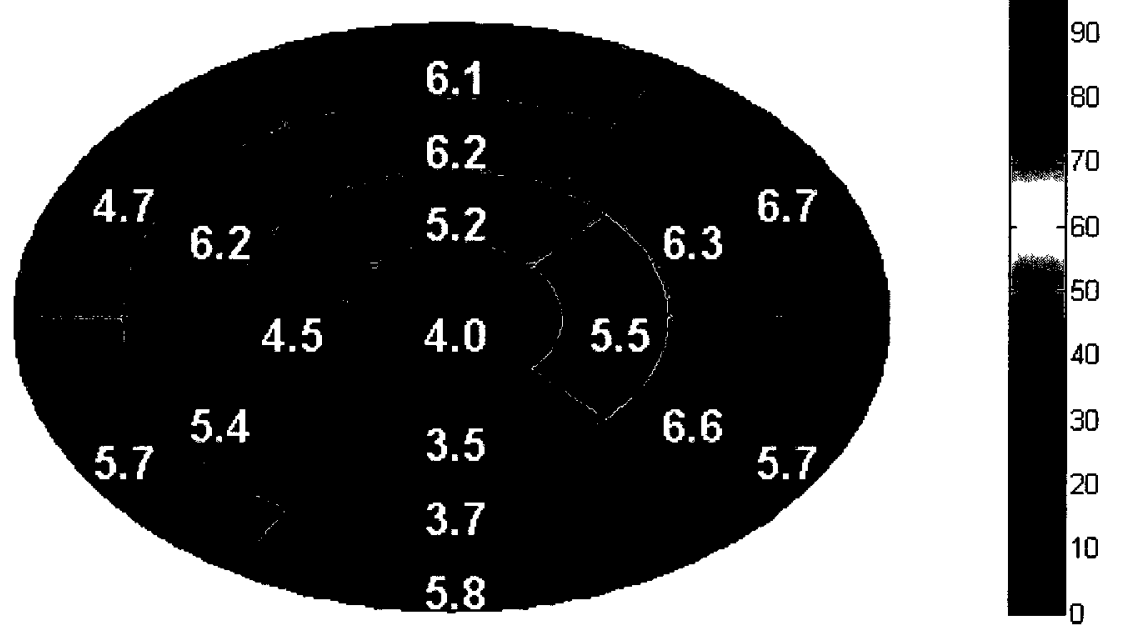

Figure 2.17: Polar map of normal database for 30 minute scan. Mean values of the uptake of tracer in each segment are indicated by the colour bar. The standard deviation in the uptake in each segment is indicated by the number overlain on that segment.

\subsubsection{Myocardial Infarct (MI) Rat}

\subsubsection{10 Minute Study}

The change of weight of the MI rats is shown in the figure 2.18. During the two weeks duration of the study the weight did not have as significant change as the normal rats. The mean weight of 8 MI rats' was $473 \pm 32 \mathrm{~g}$ while the average weight of 8 MI rats' was $446 \pm 20 \mathrm{~g}$ on day 1 and $498 \pm 32 \mathrm{~g}$ on day 14 . 


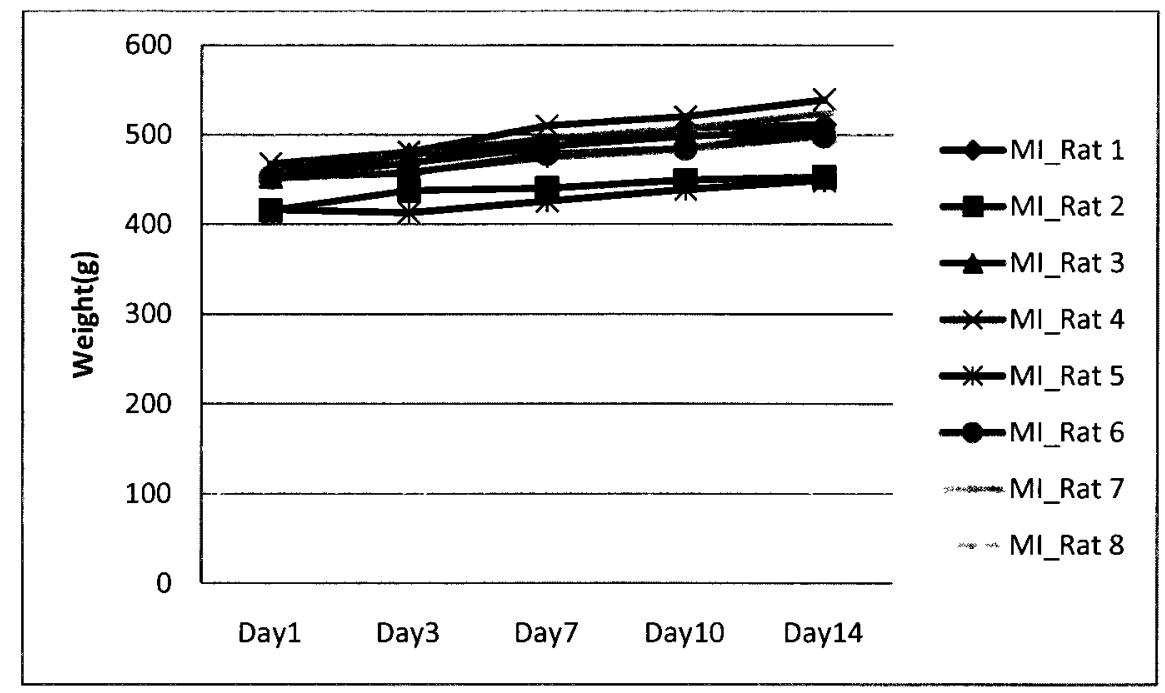

Figure 2.18: The variability of weight of MI rats during the experiment.

The reproducibility of uncorrected end diastolic volume across scans was $50 \pm$ $10 \mathrm{~mm}^{3}$ and across rats, it was $80 \pm 6 \mathrm{~mm}^{3}$. The reproducibility of uncorrected end systolic volume across scans was $20 \pm 3 \mathrm{~mm}^{3}$ and across rats was $75 \pm 5 \mathrm{~mm}^{3}$. When the left ventricular volumes were corrected, the intra-subject reproducibility of EDV and ESV was $45 \pm 8 \mathrm{~mm}^{3}$ and $20 \pm 2 \mathrm{~mm}^{3}$, respectively. The inter-subject reproducibility at that time for EDV and ESV was $79 \pm 6 \mathrm{~mm}^{3}$ and $75 \pm 4 \mathrm{~mm}^{3}$ respectively. There is no significant change observed across scans or across rats for MI rats. Figure 2.19 shows the inter- and intra-subject reproducibility of uncorrected left ventricular volumes, meanwhile figure 2.20 shows the reproducibility of corrected volumes. 
(a)

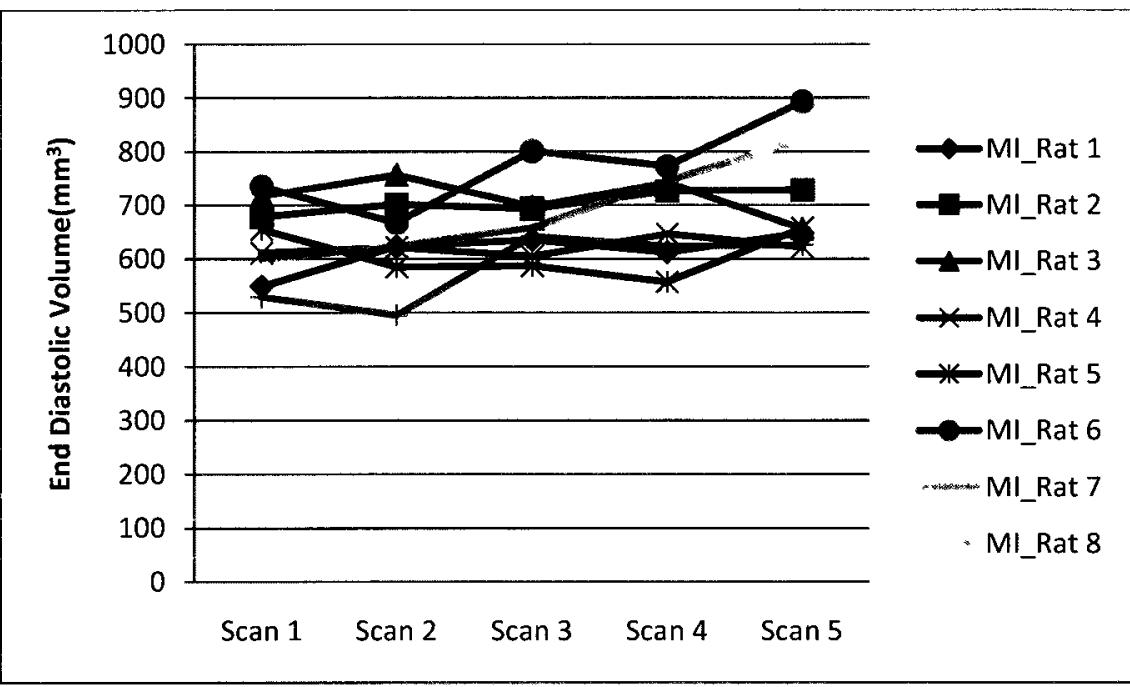

b)

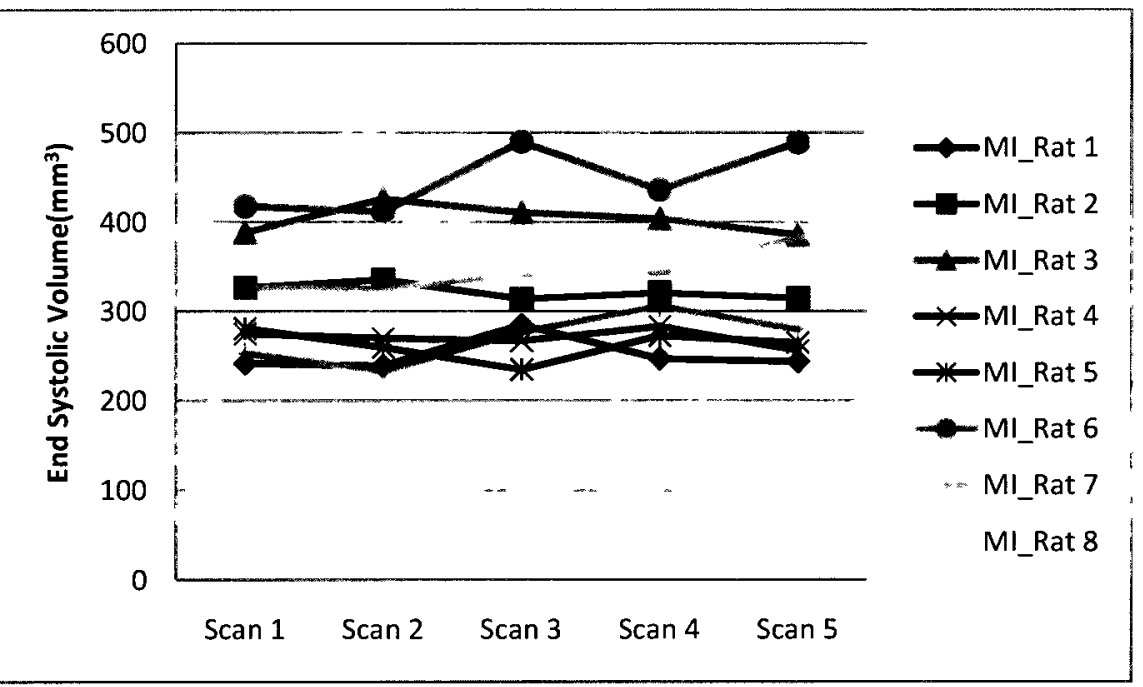

Figure 2.19: Inter- and intra-subject reproducibility of uncorrected (a) EDV and (b) ESV for a 10 minute scans of MI rats. 
(a)

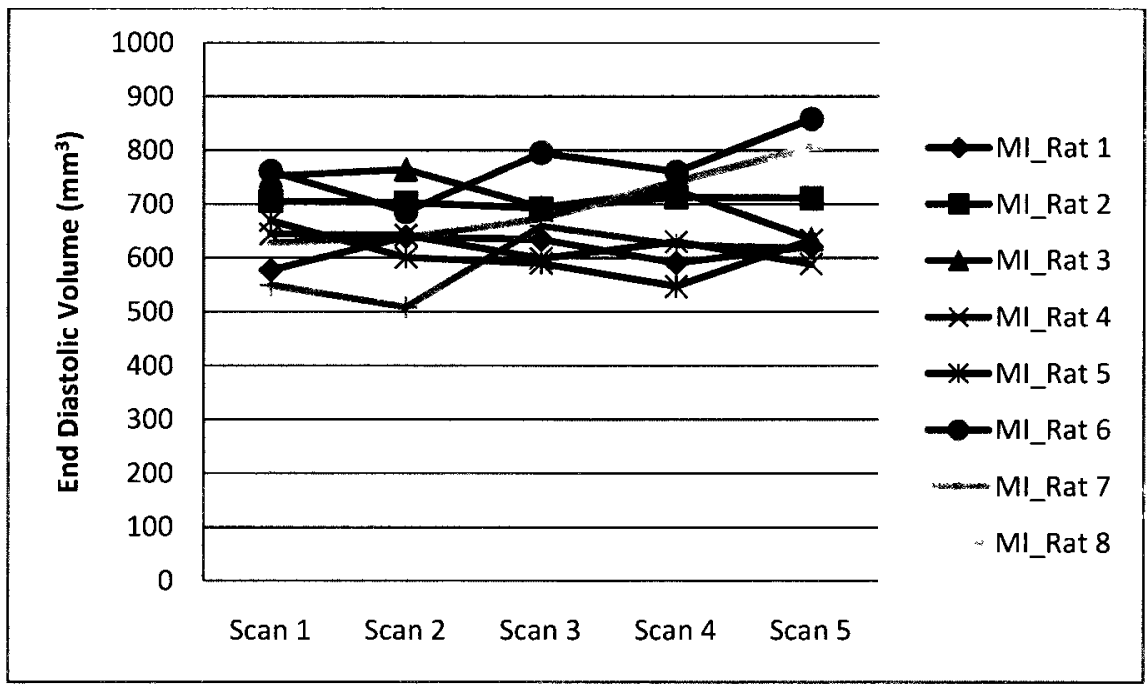

(b)

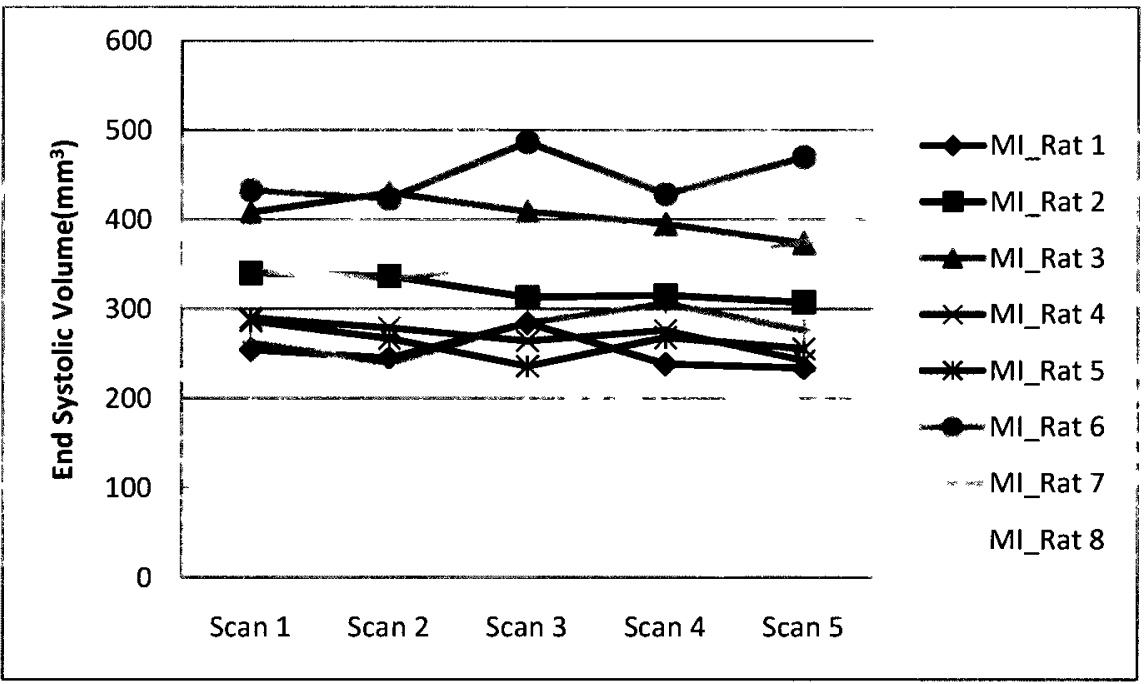

Figure 2.20: Inter- and intra- subject reproducibility of weight corrected (a) EDV and (b) ESV for a 10 minute study of 8 MI rats.

The results of the 10 minute experiment for the ejection fraction and perfusion homogeneity in the pinhole myocardial perfusion SPECT for MI rats are shown in figure 2.21 . For the EF, the reproducibility of intra subject study was $3 \pm 0.3 \%$ while for inter subject study it was $7 \pm 1 \%$. The average of PH across scans was $12 \pm 0.3$ $\%$, at the same time across rats was $12 \pm 0.4 \%$. 
(a)

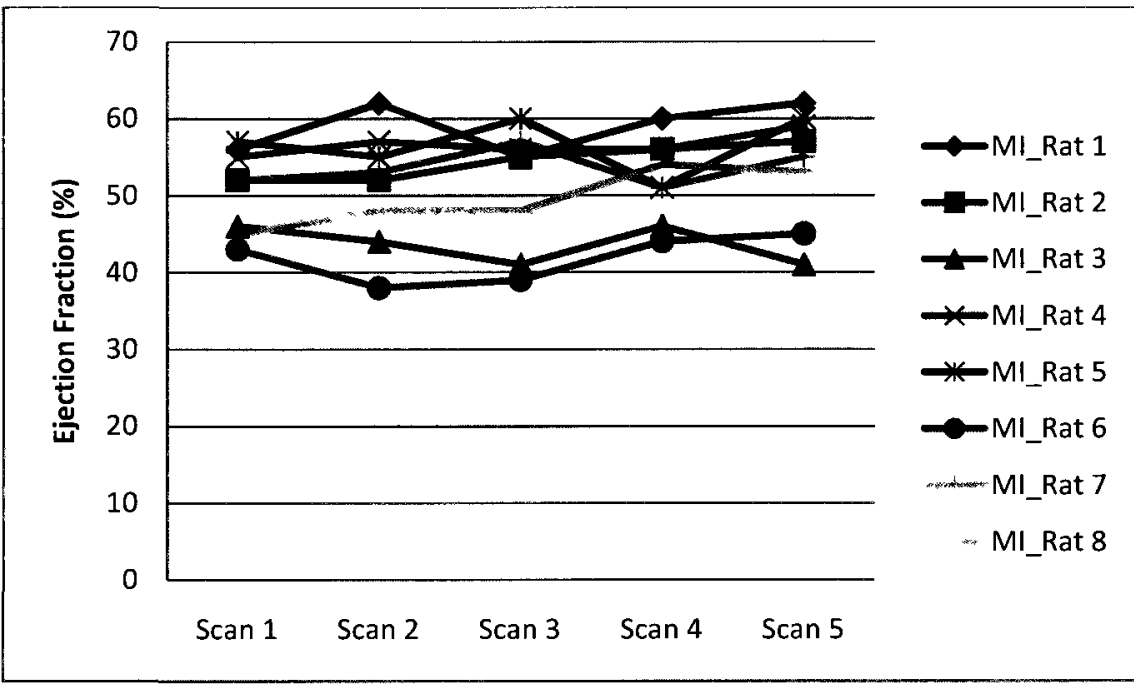

(b)

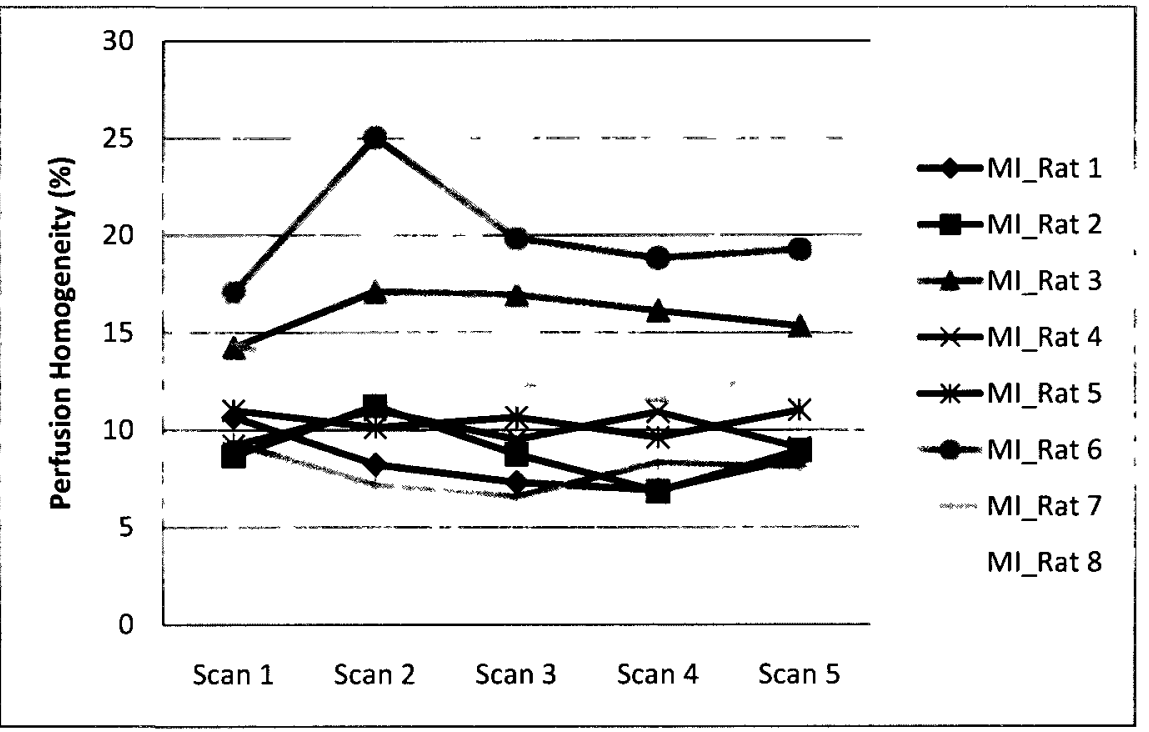

Figure 2.21: Inter- and Intra-subject reproducibility of (a) EF and (b) PH for 10 minute scan of 8 MI rats. 
The z-score for the measurement of tracer uptake for the $8 \mathrm{MI}$ rats is calculated based on the 10-minute normal database and the results are shown in figure 2.22 .

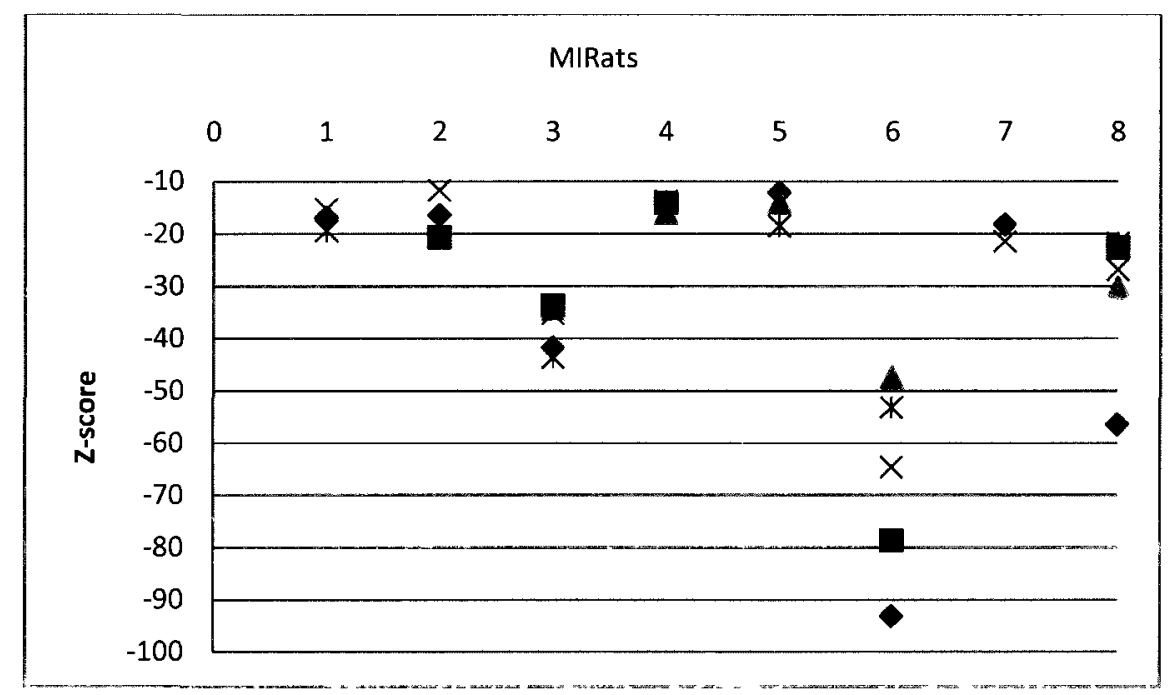

Figure 2.22: z-scores of the measurement of tracer uptake for the $8 \mathrm{MI}$ rats for the 10 minute scans. The $\mathrm{z}$-score here represents the sum of the $\mathrm{z}$-values $<-2$ over the 17 -segments of the heart, determined using the normal rat database.

\subsubsection{20 Minute Study}

The results of the left ventricular volumes, EF and PH of 20 minute scan are given now in this section. Figure 2.23 and figure 2.24 show the inter- and intrasubject reproducibility of uncorrected and corrected volumes. The intra-subject reproducibility of uncorrected EDV and ESV were $41 \pm 9 \mathrm{~mm}^{3}$ and $24 \pm 4 \mathrm{~mm}^{3}$ whereas for corrected EDV and ESV were $42 \pm 8 \mathrm{~mm}^{3}$ and $25 \pm 3 \mathrm{~mm}^{3}$, respectively. On the contrary, the inter-subject reproducibility of uncorrected EDV and ESV were 
$106 \pm 3 \mathrm{~mm}^{3}$ and $76 \pm 3 \mathrm{~mm}^{3}$ while for corrected EDV and ESV it was $106 \pm 2 \mathrm{~mm}^{3}$ and $76 \pm 4 \mathrm{~mm}^{3}$.

(a)

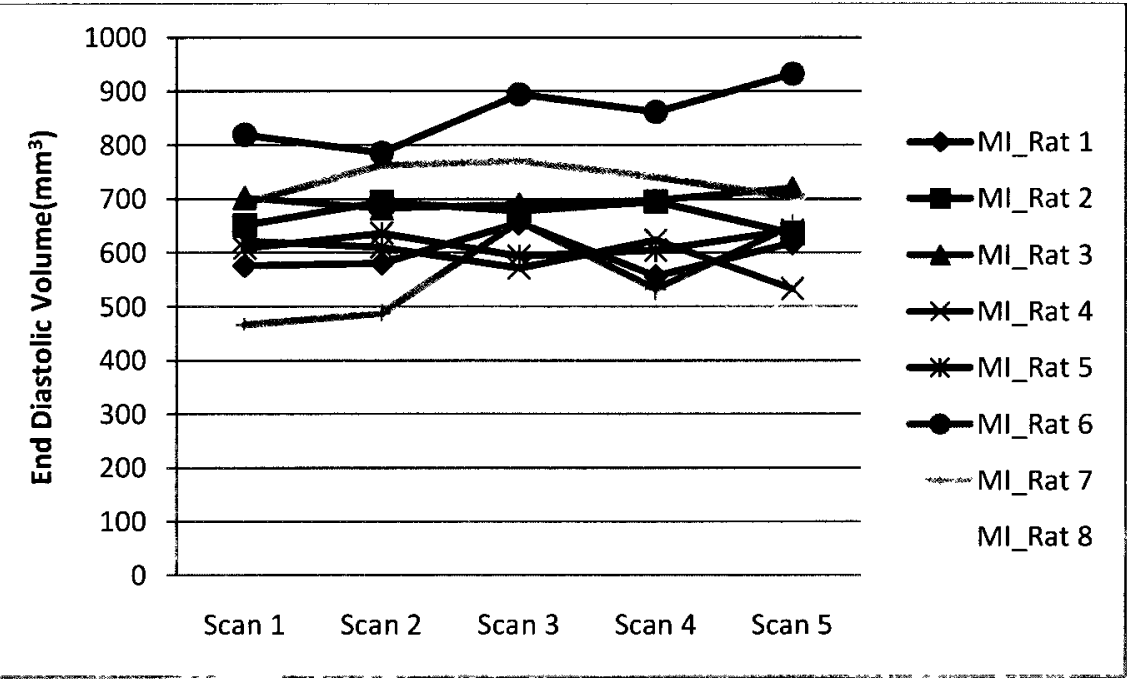

(b)

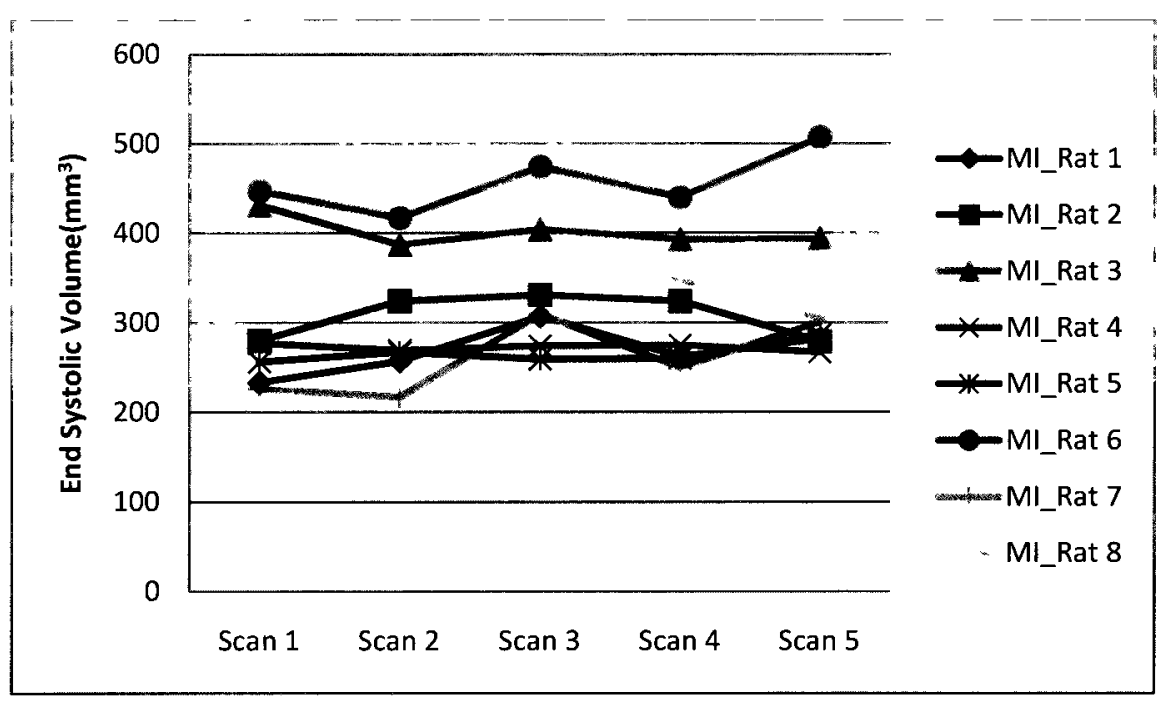

Figure 2.23: The inter- and intra-subject reproducibility of uncorrected (a) EDV and (b) ESV for 20 minute scans duration of MI rats. 

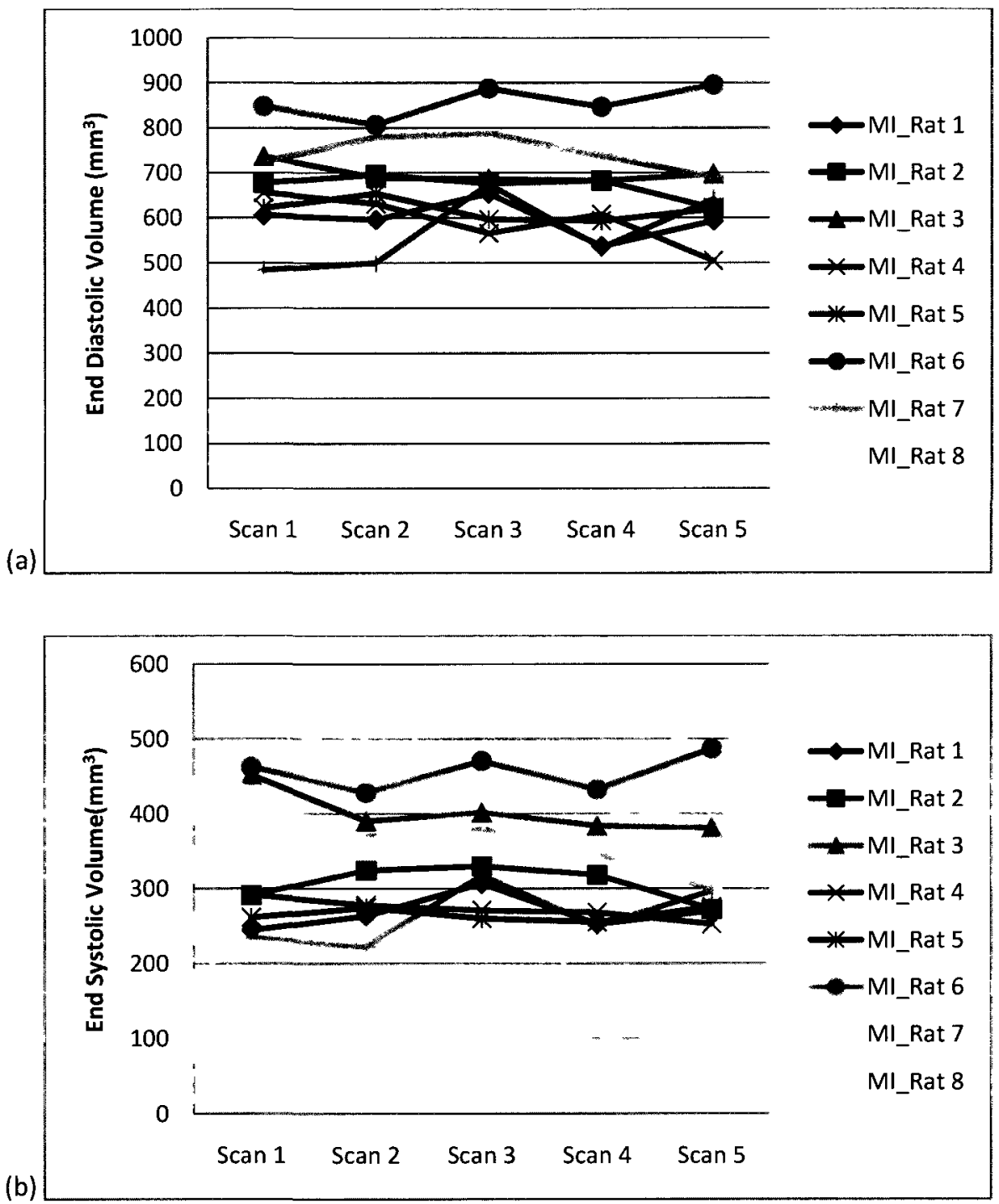

Figure 2.24: The inter- and intra-subject reproducibility of corrected (a) EDV and (b) ESV for 20 minute scans of MI rats.

The average standard deviation of the EF across scans was $2 \pm 0.2 \%$ and across rats was $5 \pm 0.5 \%$. At the same time the average $\mathrm{PH}$ across scans was $12 \pm 0.2$ $\%$ and across rats was $12 \pm 0.2 \%$. Figure 2.25 shows the reproducibility of EF and $\mathrm{PH}$ across scans and across rats. 
(a)

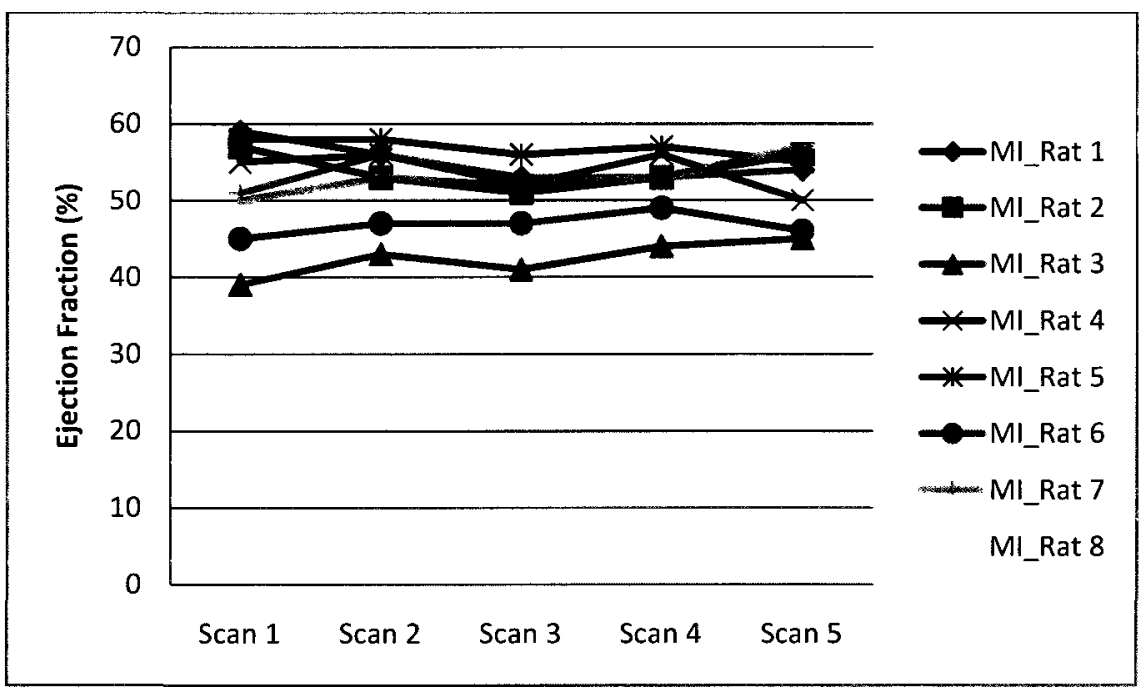

(b)

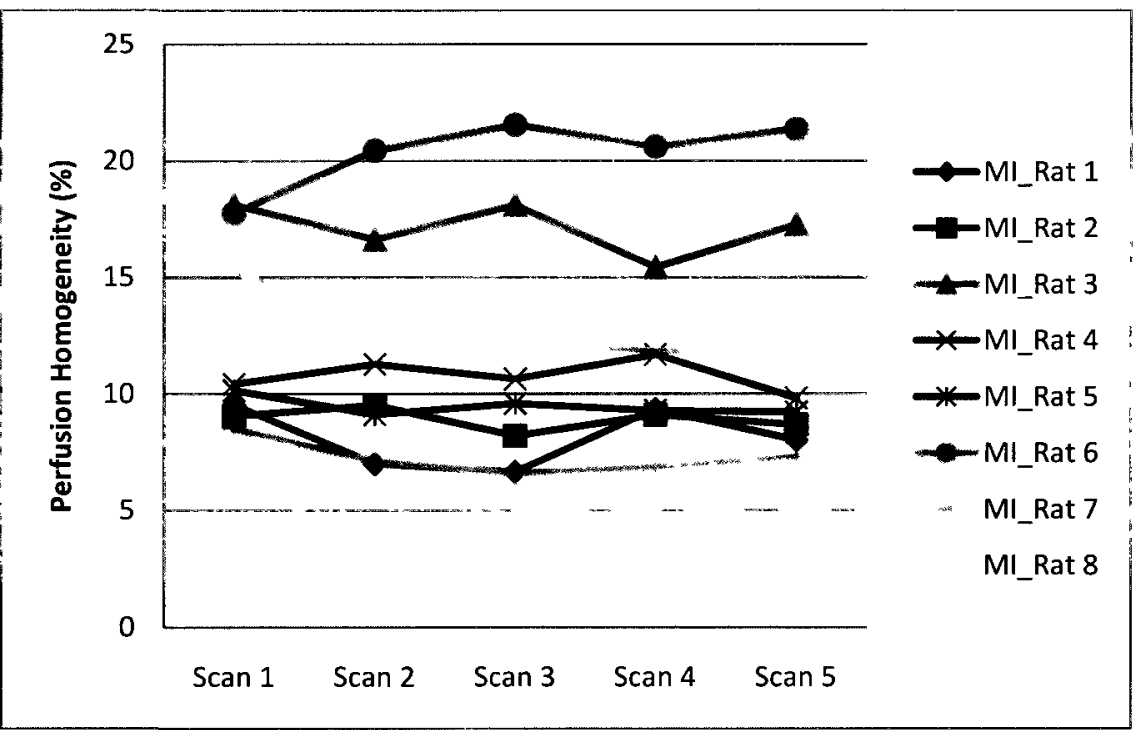

Figure 2.25: Reproducibility of (a) EF and (b) PH, across scans and across rats, for a 20 minute study of MI rats. 
In figure 2.26 we plotted the z-score for the measurement of tracer uptake for 20 minute scan of 8 MI rats.

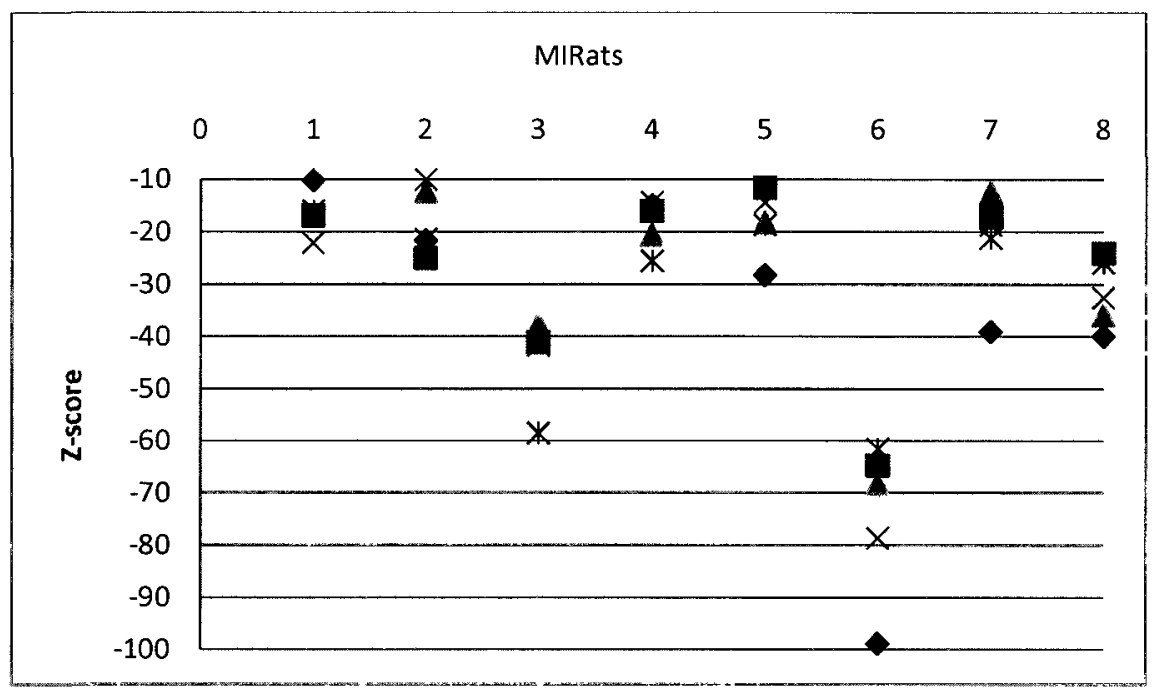

Figure 2.26: z-scores of the measurement of tracer uptake of 8 MI rats for 20 minute scans. The z-score here represents the sum of the $z$-values $<-2$ over the 17-segments of the heart, determined using the normal rat database.

\subsubsection{30 Minute Study}

The result of the investigation of pinhole gated myocardial perfusion SPECT of MI rats for the uncorrected volumes are shown in figure 2.27. The average of the standard deviation of the intra-subject EDV is $32 \pm 4 \mathrm{~mm}^{3}$ and ESV is $24 \pm 3 \mathrm{~mm}^{3}$ whereas the inter-subject reproducibility of EDV and ESV are $89 \pm 4 \mathrm{~mm}^{3}$ and $71 \pm 4$ $\mathrm{mm}^{3}$ respectively. 
(a)

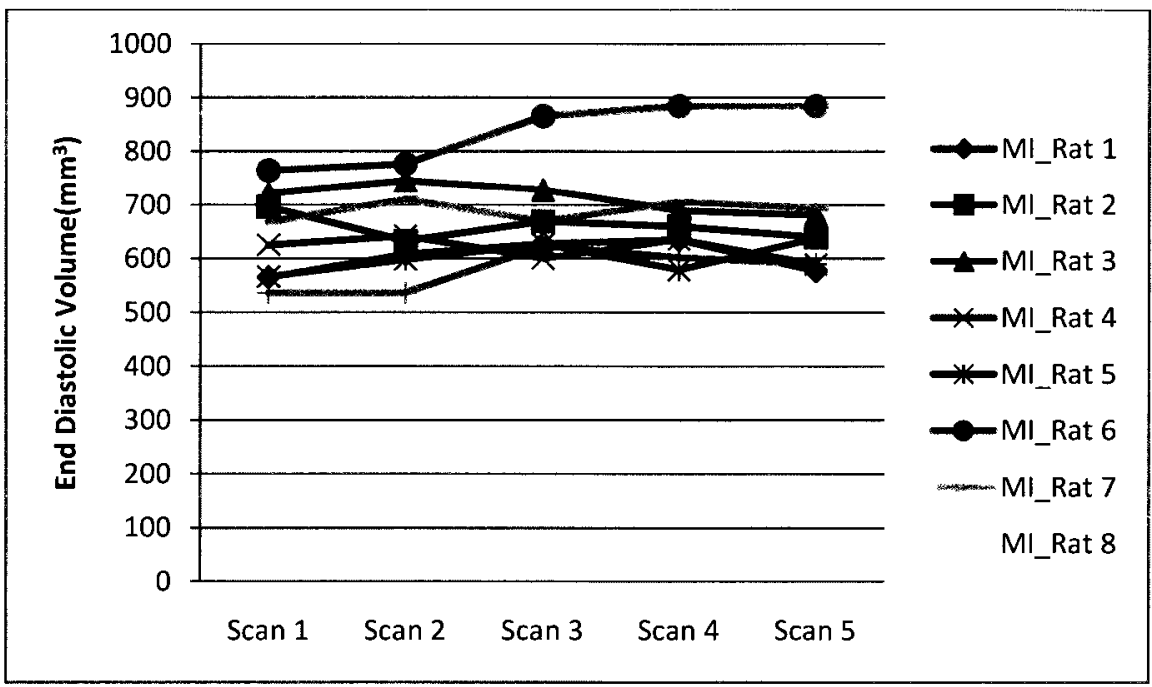

(b)

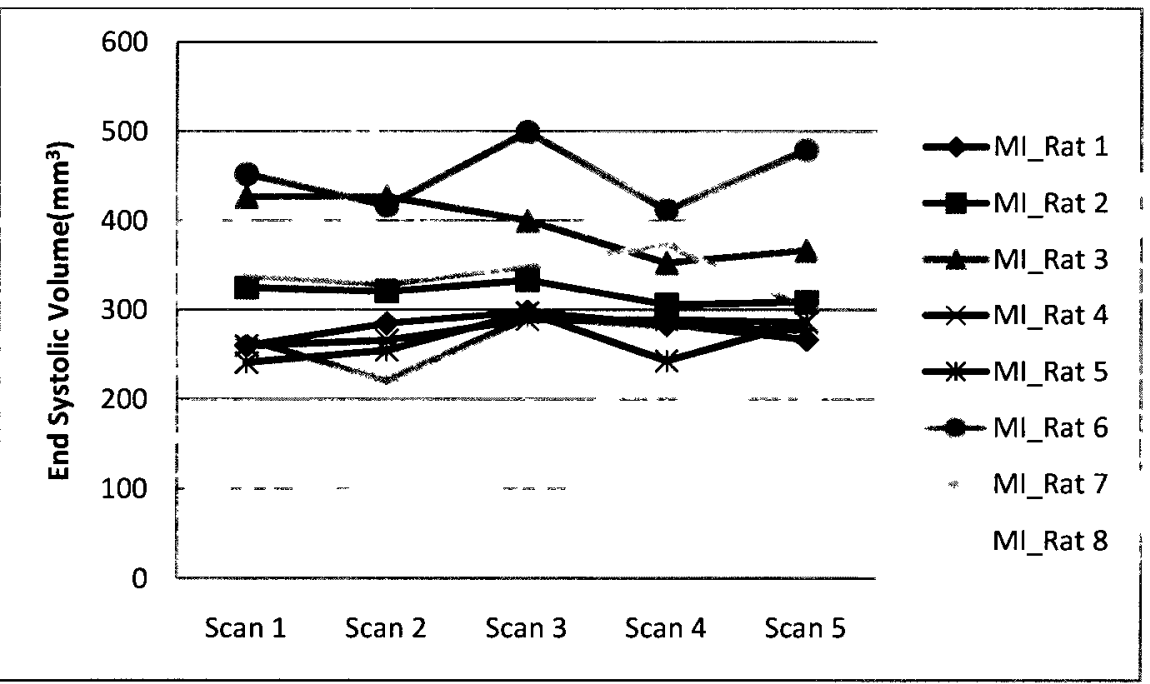

Figure 2.27: Inter- and intra-subject reproducibility of uncorrected (a) end

diastolic volume (EDV) and (b) end systolic volume (ESV) for 30 minute scans

for $8 \mathrm{MI}$ rats.

The reproducibility of the corrected EDV and ESV for intra-subject are $35 \pm 3$ $\mathrm{mm}^{3}$ and $25 \pm 4 \mathrm{~mm}^{3}$ respectively whıle for inter-subject they are $88 \pm 3 \mathrm{~mm}^{3}$ and 71 $\pm 5 \mathrm{~mm}^{3}$ respectively. Figure 2.28 shows this reproducibility of EDV and ESV. 
(a)

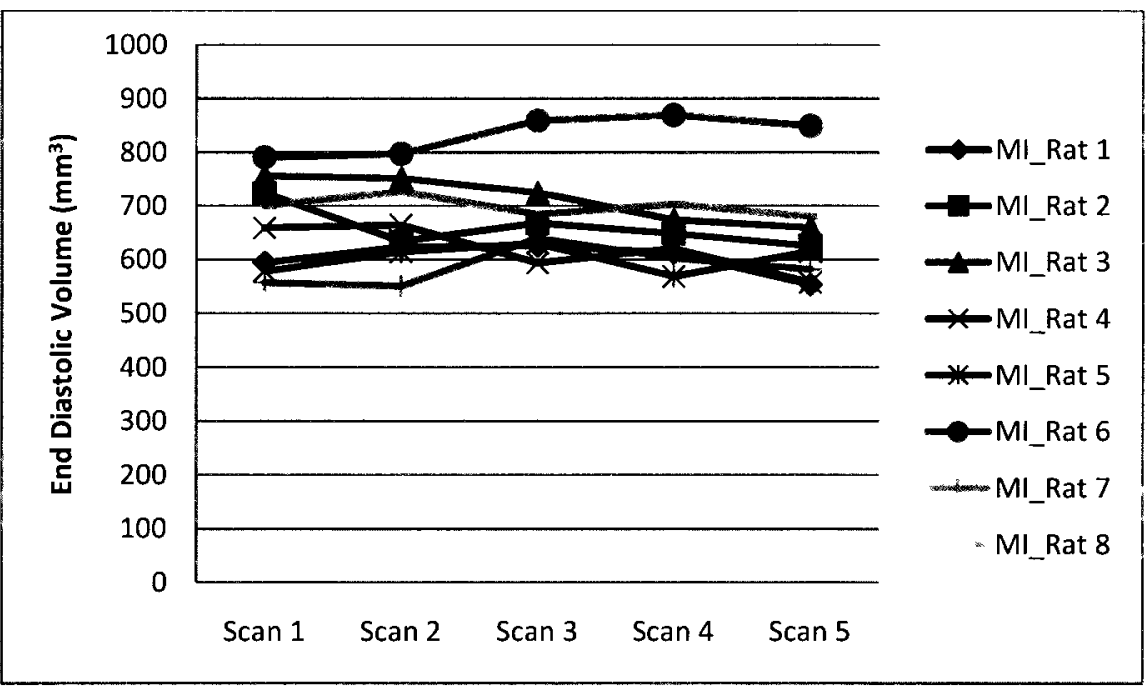

(b)

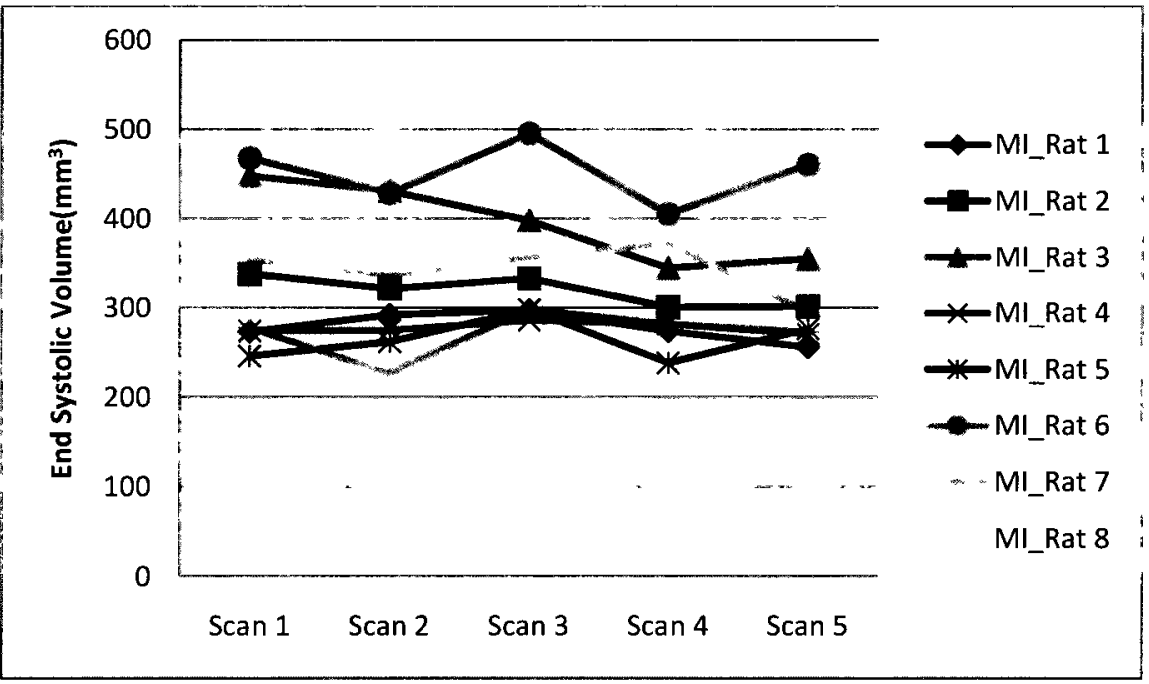

Figure 2.28: Reproducibility of corrected (a) EDV and (b) ESV for intra- and inter-subject for $\mathbf{3 0}$ minute study of MI rats.

The reproducibility of pinhole gated myocardial perfusion SPECT of ejection fraction and perfusion homogeneity are shown in figure 2.29 . The result of the EF across scans is $3 \pm 1 \%$ and across rats is $5 \pm 1 \%$ while the intra-subject reproducibility of $\mathrm{PH}$ is $12 \pm 0.1 \%$ and inter-subject is $12 \pm 0.3 \%$. 
(a)

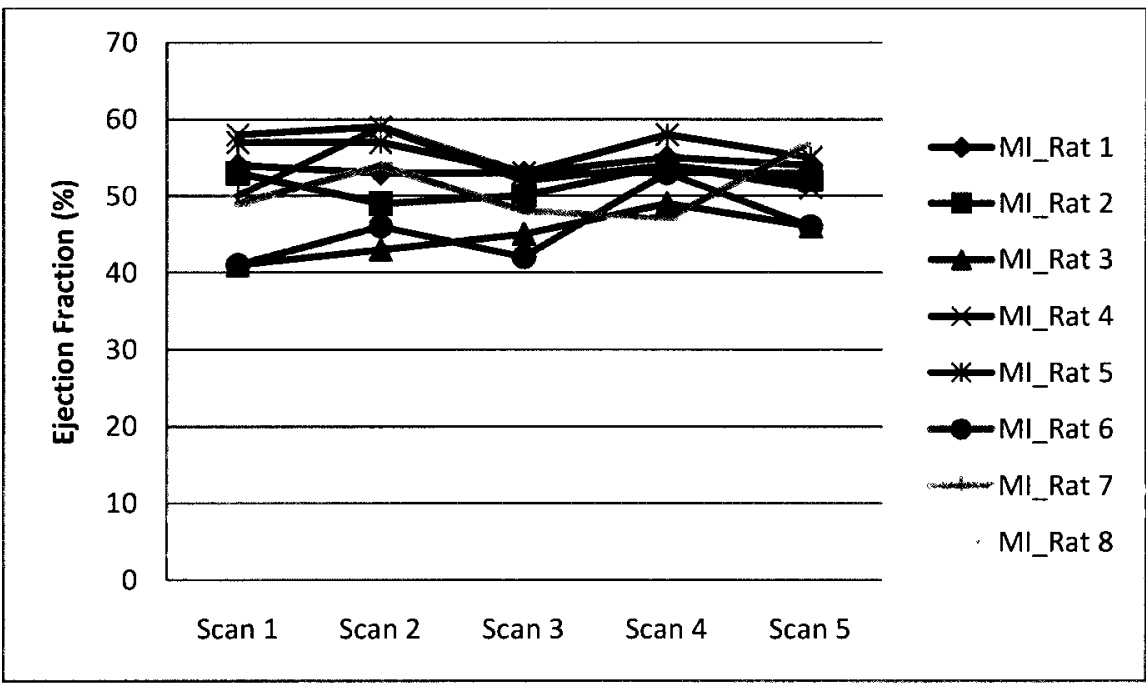

(b)

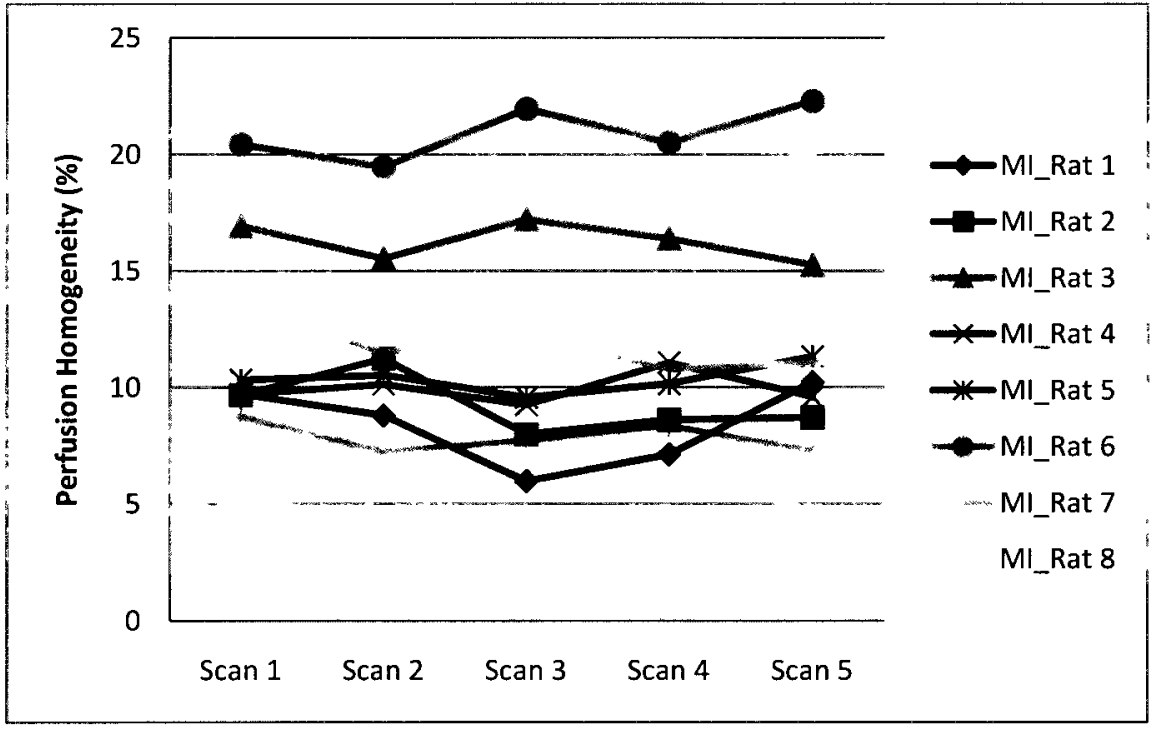

Figure 2.29: Reproducibility of intra- and inter-subject (a) EF and (b) PH for 30 minute study of MI rats. 
The z-score for the measurement of tracer uptake of MI rats for 30 minute study is shown in figure 2.30 .

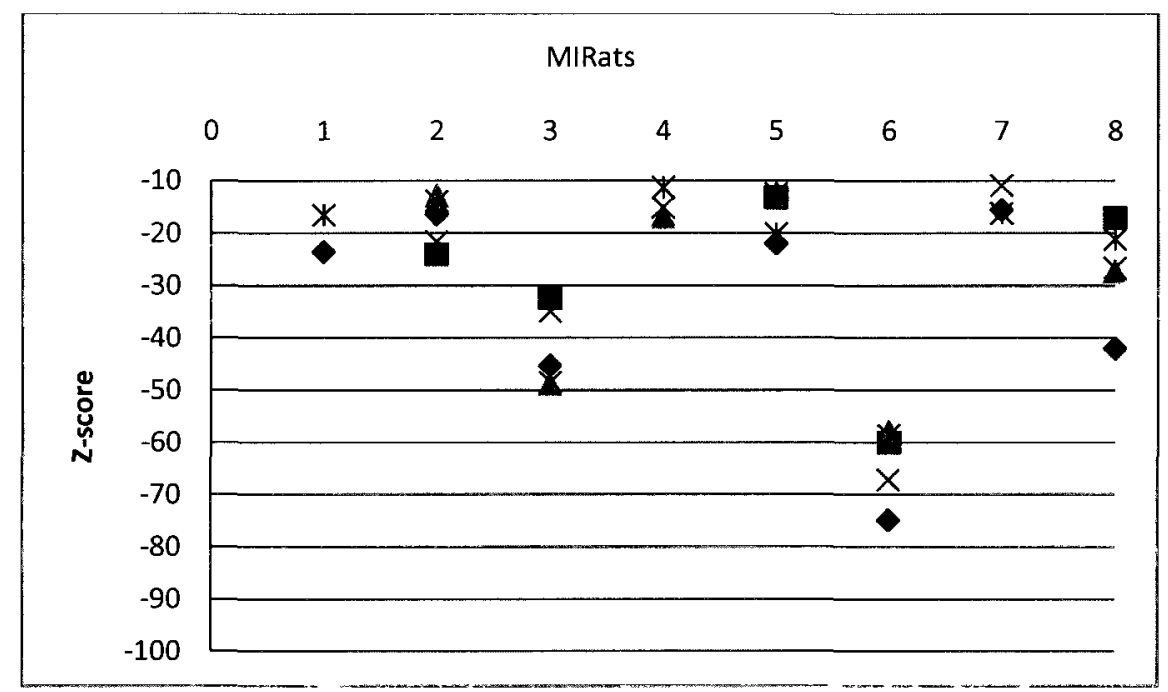

Figure 2.30: z-scores for the measurement of tracer uptake of $8 \mathrm{MI}$ rats for 30 minute study. The z-score here represents the sum of the $z$-values $<-2$ over the 17 -segments of the heart, determined using the normal rat database.

\subsubsection{Summary}

Table 2.2 summarizes all of the observed results for 10 minute, 20 minute and 30 minute scan to have an idea about the reproducibility at a glance. Table 2.3 shows the variability expressed as a percentage of the mean value of EDV and ESV of normal and MI rats for different scan duration. A sample calculation is shown in the appendix. 


\begin{tabular}{|c|c|c|c|c|c|c|}
\hline \multicolumn{7}{|c|}{ Table 2.2: Average standard deviation of observed measures } \\
\hline Time & Rat & & $\operatorname{EDV}\left(\mathrm{mm}^{3}\right)$ & $\mathbf{E S V}\left(\mathbf{m m}^{3}\right)$ & EF $(\%)$ & PH (\%) \\
\hline \multirow{4}{*}{$\begin{array}{c}10 \\
\text { minutes }\end{array}$} & \multirow{2}{*}{ Normal } & $\begin{array}{c}\text { Across } \\
\text { scans }\end{array}$ & $30 \pm 3 \dagger$ & $18 \pm 2$ & $2 \pm 0.2 \dagger$ & $7 \pm 0.1 \ddagger$ \\
\hline & & $\begin{array}{c}\text { Across } \\
\text { rats }\end{array}$ & $45 \pm 5 \dagger \ddagger$ & $29 \pm 5 \ddagger$ & $4 \pm 1 \dagger+$ & $7 \pm 0.1 \ddagger$ \\
\hline & \multirow{2}{*}{ MI } & $\begin{array}{l}\text { Across } \\
\text { scans }\end{array}$ & $45 \pm 8 \dagger$ & $20 \pm 2 \dagger$ & $3 \pm 0.3 \dagger$ & $12 \pm 0.3 \ddagger$ \\
\hline & & $\begin{array}{c}\text { Across } \\
\text { rats }\end{array}$ & $79 \pm 6+\ddagger$ & $75 \pm 4 \dagger \ddagger$ & $7 \pm 0.5 \dagger \ddagger$ & $12 \pm 0.4 \neq$ \\
\hline \multirow{4}{*}{$\begin{array}{c}20 \\
\text { minutes }\end{array}$} & \multirow{2}{*}{ Normal } & $\begin{array}{l}\text { Across } \\
\text { scans }\end{array}$ & $25 \pm 3$ & $15 \pm 2 \ddagger$ & $2 \pm 0.1 \dagger$ & $7 \pm 0.1 \div$ \\
\hline & & $\begin{array}{c}\text { Across } \\
\text { rats }\end{array}$ & $38 \pm 5 \ddagger$ & $23 \pm 4 \ddagger$ & $3 \pm 0.1 \uparrow+$ & $7 \pm 0.2 \div$ \\
\hline & \multirow{2}{*}{ MI } & $\begin{array}{l}\text { Across } \\
\text { scans }\end{array}$ & $42 \pm 8 \dagger$ & $25 \pm 3+\div$ & $2 \pm 0.2 \uparrow$ & $12 \pm 0.2 \div$ \\
\hline & & $\begin{array}{c}\text { Across } \\
\text { rats }\end{array}$ & $106 \pm 2+t$ & $76 \pm 4+\div$ & $5 \pm 0.5+t$ & $12 \pm 0.2 \div$ \\
\hline \multirow{4}{*}{$\begin{array}{c}30 \\
\text { minutes }\end{array}$} & \multirow{2}{*}{ Normal } & $\begin{array}{l}\text { Across } \\
\text { scans }\end{array}$ & $29 \pm 4$ & $15 \pm 2 \dagger$ & $2 \pm 0.3 \dagger$ & $7 \pm 0.2 \neq$ \\
\hline & & $\begin{array}{c}\text { Across } \\
\text { rats }\end{array}$ & $38 \pm 3 \div$ & $28 \pm 2 \dagger+$ & $3 \pm 0.3 \dagger$ & $7 \pm 0.3 \ddagger$ \\
\hline & \multirow{2}{*}{ MI } & $\begin{array}{l}\text { Across } \\
\text { scans }\end{array}$ & $35 \pm 3 \dagger$ & $25 \pm 4 \dagger$ & $3 \pm 0.5 \dagger$ & $12 \pm 0.1 \ddagger$ \\
\hline & & $\begin{array}{c}\text { Across } \\
\text { rats }\end{array}$ & $88 \pm 3+\neq$ & $71 \pm 5 \dagger \ddagger$ & $5 \pm 0.6 \dagger$ & $12 \pm 0.3 \ddagger$ \\
\hline
\end{tabular}

$\uparrow$ Intra- and inter-rat values are significantly different $(\mathrm{p}<0.05)$

$\$$ Normal and Myocardial Infarct rat values are significantly different $(\mathrm{p}<0.05)$ 
Table 2.3: Variability as a percentage of EDV and ESV.

\begin{tabular}{|c|c|c|c|c|}
\hline Time & Rat & Intra/Inter & EDV (\%) & ESV (\%) \\
\hline \multirow{3}{*}{10 minute } & \multirow{3}{*}{ Normal } & Intra rat & 6.4 & 8.6 \\
\cline { 3 - 5 } & & Inter rat & 9.6 & 14.4 \\
\cline { 3 - 5 } & \multirow{3}{*}{ MI } & Intra rat & 6.7 & 6.3 \\
\cline { 3 - 5 } & & Inter rat & 11.9 & 23.1 \\
\hline \multirow{3}{*}{20 minute } & \multirow{3}{*}{ Normal } & Intra rat & 5.7 & 7.3 \\
\cline { 3 - 5 } & & Inter rat & 8.4 & 11.4 \\
\cline { 3 - 5 } & \multirow{3}{*}{ MI } & Intra rat & 6.4 & 7.7 \\
\cline { 3 - 5 } & \multirow{3}{*}{ Normal } & Inter rat & 15.9 & 23.6 \\
\cline { 3 - 5 } & & Intra rat & 6.3 & 7.2 \\
\cline { 3 - 5 } & \multirow{3}{*}{ MI } & Inter rat & 8.4 & 13.2 \\
\cline { 3 - 5 } & & Intra rat & 5.2 & 7.7 \\
\hline & & Inter rat & 13.3 & 21.8 \\
\hline
\end{tabular}

\subsubsection{Comparison}

\subsubsection{Comparison between Tc99 ${ }^{\mathrm{m}}$-tetrofosmin and Tl-201 Study}

Though the injected activities are different, our measurements of cardiac function with Tl-201 for normal rats, across scans and across rats, are similar to those obtained with $\mathrm{Tc} 99 \mathrm{~m}$-tetrofosmin ( $2 \mathrm{mCi}$ injection). This suggests that perfusion imaging with Tl-201 may be used as an alternative to Tc99m-tracers without significant loss in precision. The comparison of that study (Strydhorst, J 2010) with our study is given below: 


\begin{tabular}{|c|c|c|c|c|c|}
\hline \multicolumn{6}{|c|}{$\begin{array}{r}\text { Table 2.4: Comparison of our Tl-201 study } \\
\text { study }\end{array}$} \\
\hline & & $\operatorname{EDV}\left(\mathrm{mm}^{3}\right)$ & $\operatorname{ESV}\left(\mathrm{mm}^{3}\right)$ & $\mathrm{EF}(\%)$ & $\mathrm{PH}(\%)$ \\
\hline \multirow{2}{*}{$\begin{array}{l}\text { Inter scans } \\
\text { (five scans) } \\
\text { comparison }\end{array}$} & Tl-201 & $29 \pm 4$ & $15 \pm 2$ & $2 \pm 0.3$ & $7 \pm 0.2$ \\
\hline & Tc99m & $24 \pm 10$ & $18 \pm 8$ & $3.1 \pm 1.6$ & \\
\hline \multirow{2}{*}{$\begin{array}{l}\text { Inter rats (8 } \\
\text { normal rats) } \\
\text { comparison }\end{array}$} & Tl-201 & $38 \pm 3$ & $28 \pm 2$ & $3 \pm 0.3$ & $7 \pm 0.3$ \\
\hline & Tc99m & $43 \pm 7$ & $24 \pm 4$ & $3.3 \pm 1.5$ & \\
\hline
\end{tabular}

No significant differences were found between the left ventricular volumes and ejection fraction measurements on the two different studies with different isotopes Tc99m and $\mathrm{Tl}-201 .(\mathrm{p}>0.05)$

\subsubsection{Comparison between Scan Durations for Normal Rats}

In our experiment the total scan duration was 30 minutes. But we did the whole scan in three 10 minute slots. Figure 2.31 (a), (b), (c) and (d) show the comparison of cardiac factors in between the three different time durations: 10 minute scan, 20 minute scan and 30 minute scan. Performing an ANOVA on the results from the different time durations showed no significant differences for EDV, ESV, EF and $\mathrm{PH}$. 


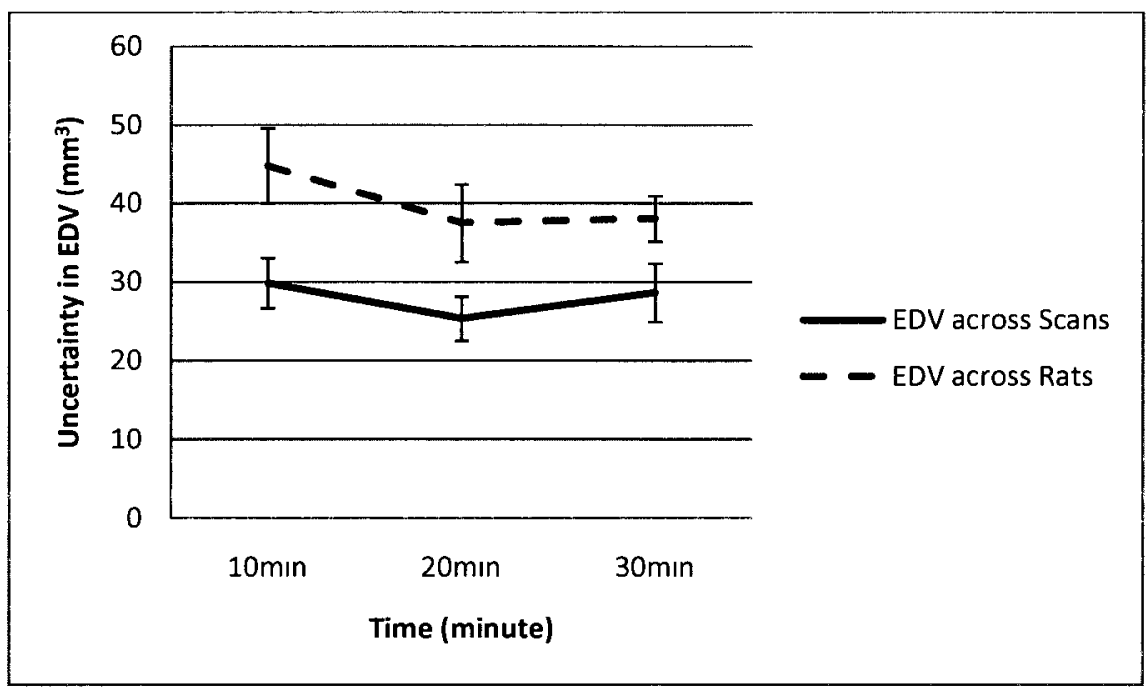

Figure 2.31(a): Comparison of time duration for the uncertainty in the end diastolic volume (EDV) measurement of normal rats.

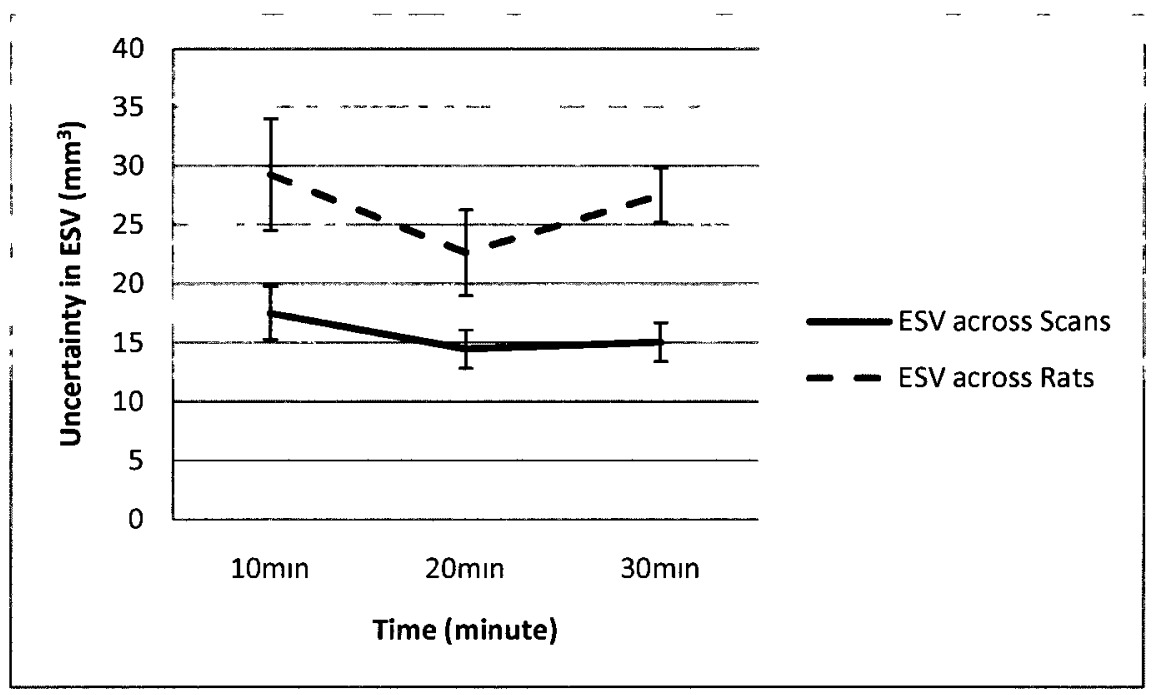

Figure 2.31(b): Comparison for the uncertainty in the end systolic volume (ESV) measurement of normal rats. 


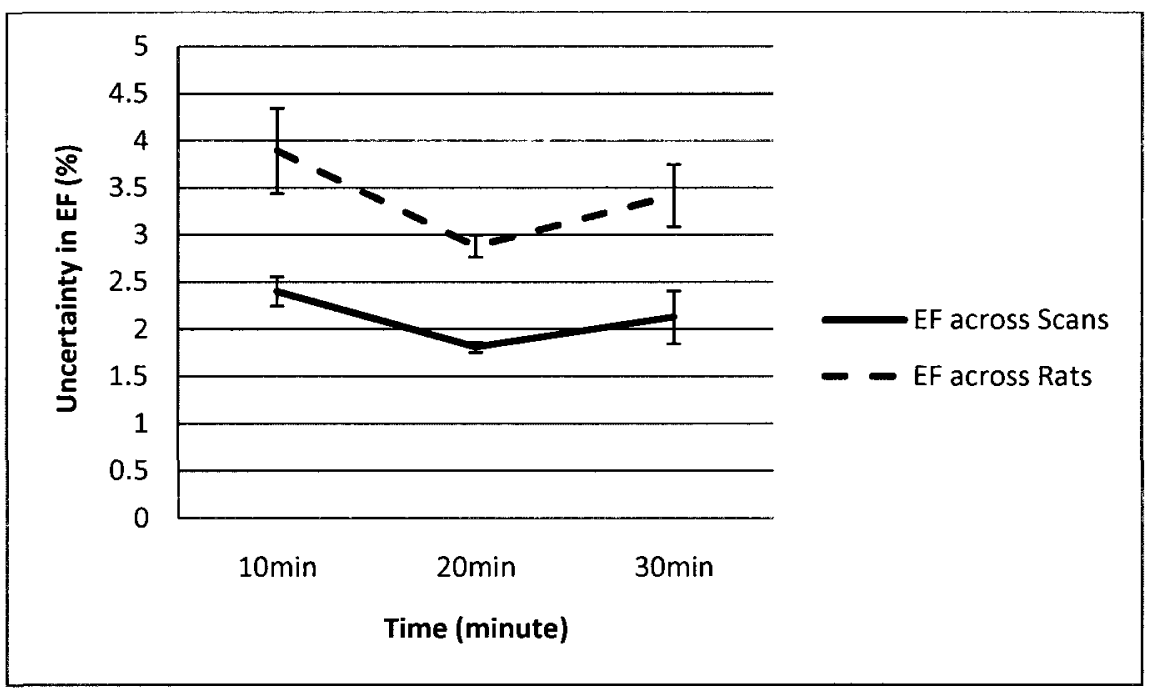

Figure 2.31(c): Comparison for the uncertainty in Ejection

fraction (EF) measurement for three different time duration of normal

rats.

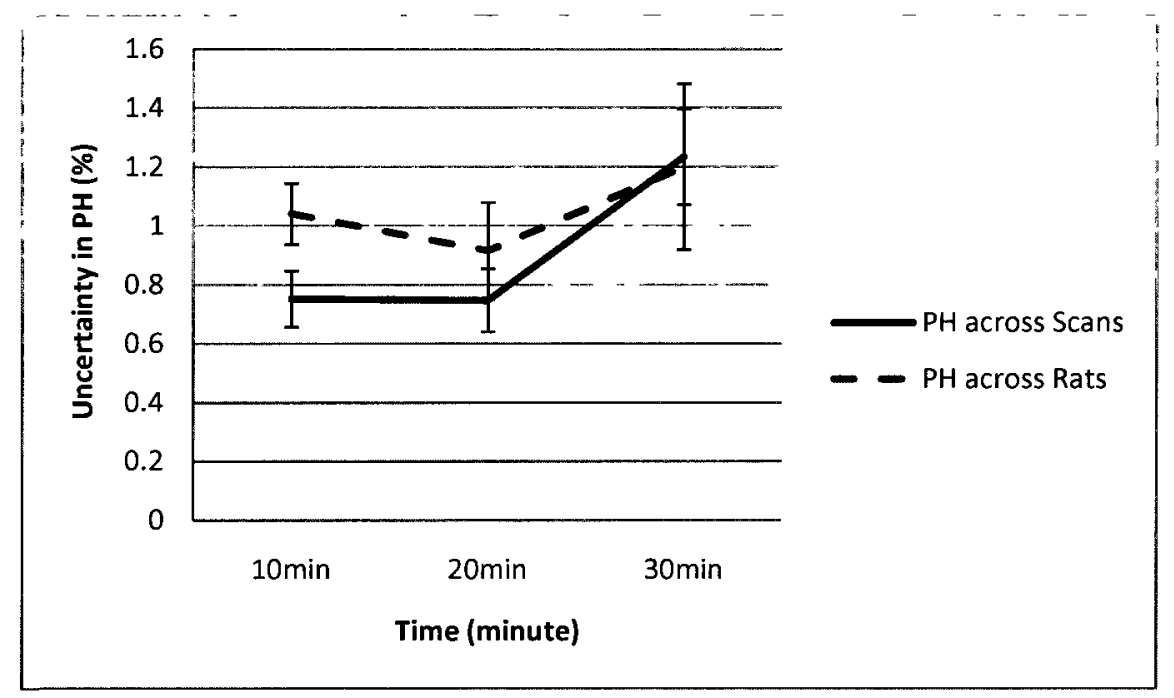

Figure 2.31(d): Comparison for the uncertainty in perfusion homogeneity (PH) measurement of normal rats. 
Although the differences are not significant, we note that there is a trend for the precision of the 20 minute scans to be better than either the 10 minute or 30 minute scans.

\subsubsection{Comparison between Scan Durations for MI Rats}

The comparisons for MI rats for different time duration are shown in this section. Figure 2.32 (a), (b), (c) and (d) show the comparison for EDV, ESV, EF and $\mathrm{PH}$ for different time duration. There were no significant differences in between 10 minute, 20 minute and 30 minute scan duration of EDV, ESV, EF and $\mathrm{PH}$.

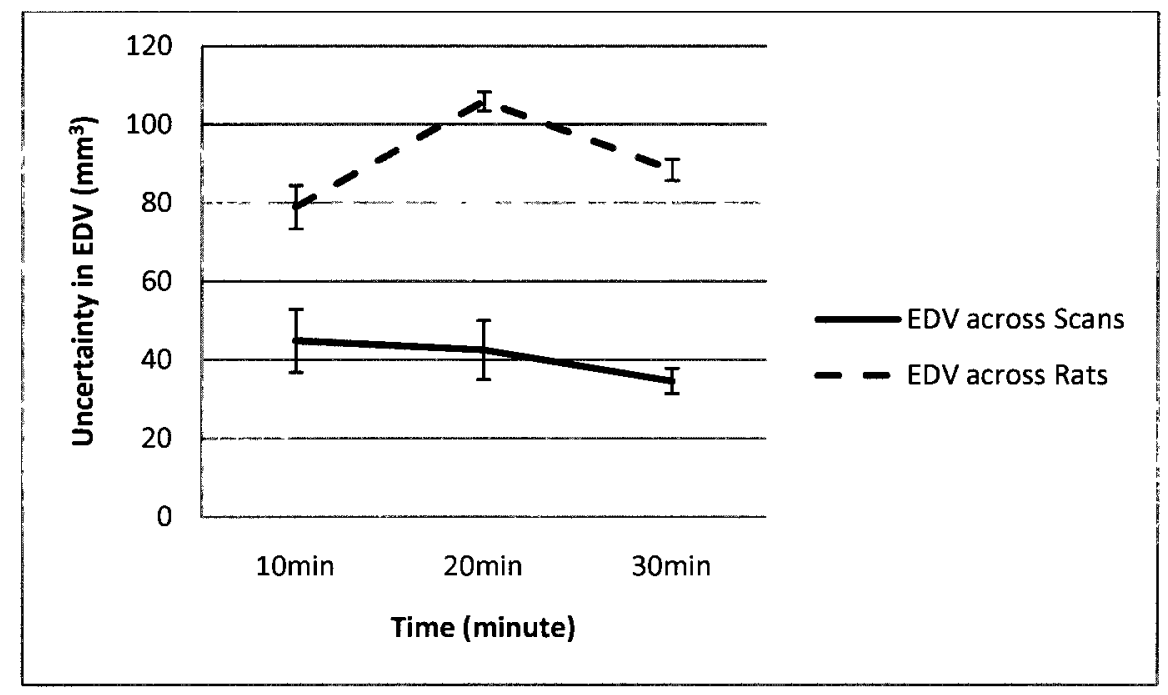

Figure 2.32(a): Comparison for the uncertainty in the EDV measurement of three different time duration of MI rats. 


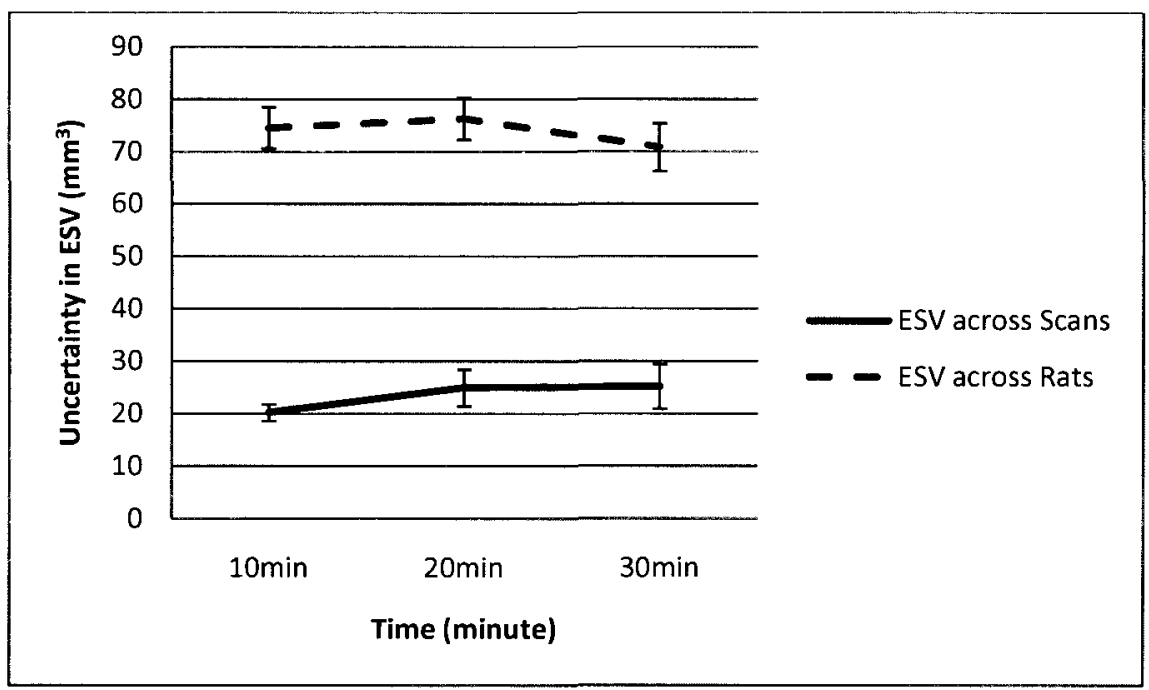

Figure 2.32(b): Comparison for the uncertainty in the ESV of three different time duration of MI rats.

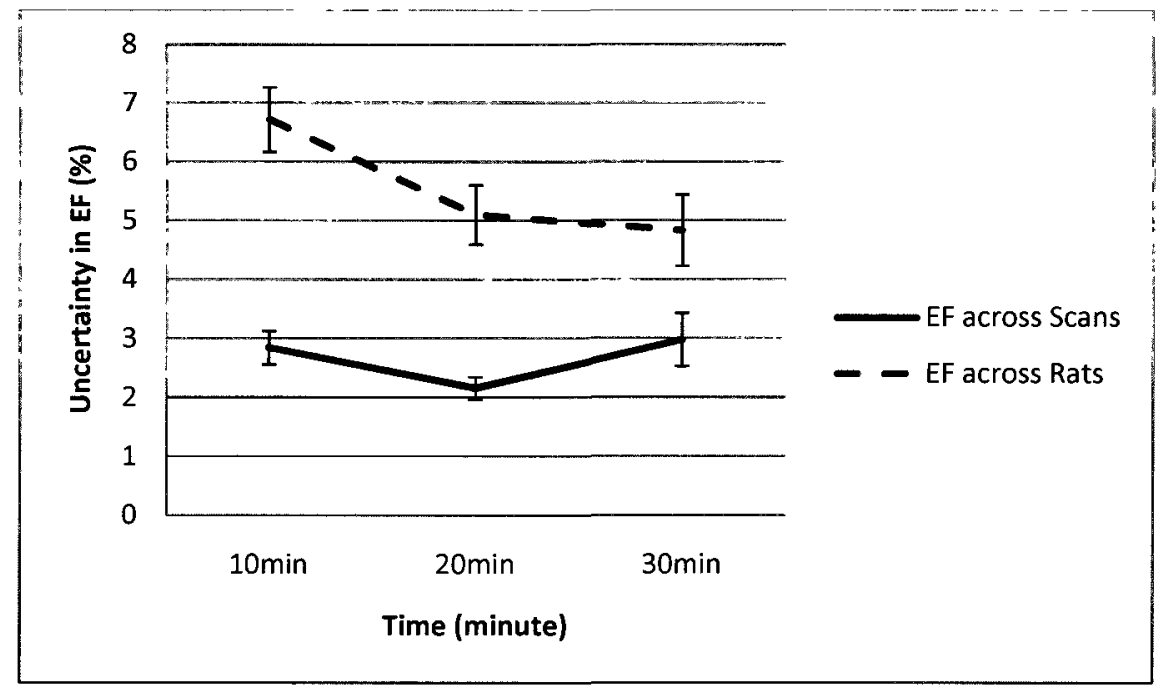

Figure 2.32(c): Comparison for the uncertainty in the EF measurement of three different time duration of MI rats. 


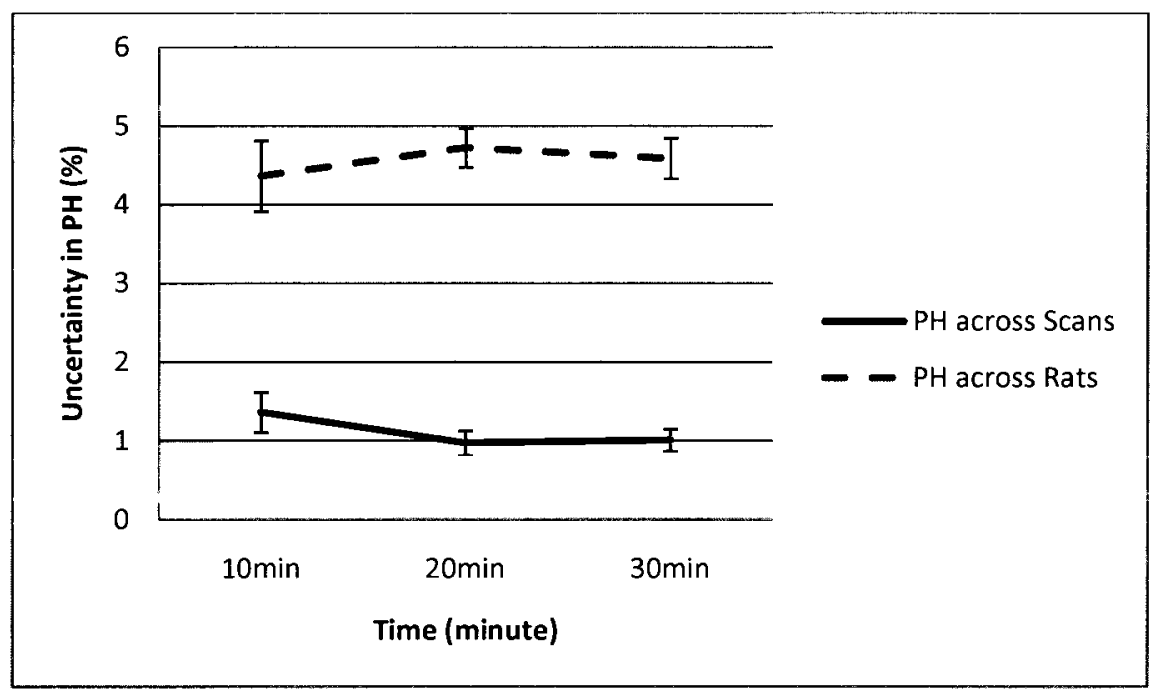

Figure 2.32(d): Comparison for the uncertainty in the PH measurement of three different time duration of MI rats.

For the MI rats, there was no significant difference between the 10,20 and 30 min duration results for either the intra- or inter-subject variability.

\subsection{Discussion}

From our results we see that the intra-subject variability is smaller than the inter-subject variability. Table 2.2 represents the result of the standard deviation for intra-(across scans) and inter-subject (across rats) reproducibility. For example, the variability for 30 minute scan duration of cardiac factors were $29 \pm 4 \mathrm{~mm}^{3}$ (EDV), 15 $\pm 2 \mathrm{~mm}^{3}(\mathrm{ESV})$ and $2 \pm 0.3 \%$ (EF) across scans while across rats the parameters were $38 \pm 3 \mathrm{~mm}^{3}(\mathrm{EDV}), 28 \pm 2 \mathrm{~mm}^{3}$ (ESV) and $3 \pm 0.3 \%$ (EF) for normal rats. The variability was higher in different rats than in the same rat which reflects the strength of repetitive imaging. For MI rats and for the same scan duration, the intra-subject reproducibility are $35 \pm 3 \mathrm{~mm}^{3}$ (EDV), $25 \pm 4 \mathrm{~mm}^{3}$ (ESV) and $3 \pm 1 \%$ (EF) whereas the inter-subject variability were $88 \pm 3 \mathrm{~mm}^{3}$ (EDV), $71 \pm 5 \mathrm{~mm}^{3}$ (ESV) and $5 \pm 1 \%$ 
(EF). The normal rats' and the MI rats' results do not show any significant differences in between the repetitive measurements $(p>0.05)$. But there was significant differences in between inter rats' reproducibility $(\mathrm{p}<0.05)$. The physiology of the animal is different for different rats. Rats differ in respiratory rate, heart rate, heart condition and biologic metabolism. Thus, the uptake of the tracer could be different for different rats. For MI rats a cause also would be the permanent ligation process. The small size of the rat heart makes it difficult to accurately reproduce the surgical infarct model from rat to rat. Also coronary trees are different from animal to animal and the response of each animal to infarcts is different. These effects combine to make a difference between different MI rats.

We represent the variability for EDV and ESV for 10 minute, 20 minute and 30 minute scanning, in a percentage form also in table 2.3. For normal rats, as a percent of the left ventricular volume, the intra-subject standard deviation (for 30 minute scan duration) was $7.2 \%(\mathrm{ESV})$ and $6.3 \%(\mathrm{EDV})$ and the inter-subject standard deviation was $13.2 \%(\mathrm{ESV})$ and $8.4 \%(\mathrm{EDV})$; the corresponding results were $7.7 \%, 5.2 \%, 21.8 \%$ and $13.3 \%$ for MI rats. The intra-rat variability was not significantly different for normal and MI rats for percent volume and EF but it was significant for inter-rat $(\mathrm{p}<0.05)$. There was also a trend to higher inter-rat than intrarat variability for EDV, ESV and EF of normal and MI rats, which supports the strength of serial imaging of small animals with Tl-201.

From our experiment, we have the result of cardiac factors for three different time durations. For normal and MI rats the comparison of these three time durations suggests the preferred length of scanning. For normal rats we see from the comparison of section 2.3.4.2 that all the 20 minute results (EDV, ESV, EF and PH) appear to have more precision than the 30 minute scans for both intra-and inter-rat variability, 
whereas the 10 minute scan and the 30 minute scan are nearly the same in precision. Our expectation was that 30 minute scanning would have more precision but that was not the case. The differences were not big enough, however, to achieve statistical significance implying that these apparent differences in reproducibility could come from the uncertainty in our measurements only. Therefore, based on our data, there is no improvement in variability between 10 and 30 minutes of acquisition. There was no statistically significant difference in between the precision of 10 minute and 20 minute scan duration. This suggests an optimal choice of 10 minute scan duration because there will not be a significant amount of gain to spend more time imaging. This result, while at first surprising, may be explained by the pharmacokinetics of Tl201 in the rat. The tracer rapidly leaves the heart such that there is not sufficient activity to improve the signal from the heart after $10 \mathrm{~min}$. Nevertheless, despite a lack of statistical significance, we did note that there seemed to be a very small but consistent improvement at 20 minute scan duration. Thus, there might be a small gain and there is no detriment to using a scan time of $20 \mathrm{~min}$. The increase of 10 extra minutes is not a large increase in imaging time. Unless there is a need to maximize the number of scans possible in a day, a $20 \mathrm{~min}$ scan may be beneficial $(10 \mathrm{~min} / \mathrm{scan}$ over a day is enough to allow an extra imaging session - allowing for time to load and unload the animal, position the animal and set up the acquisition). Thus we can say that the timing depends somewhat on the demands of the particular experiment and in general 10 or 20 minute would be the best scanning time.

We did not evaluate shorter imaging intervals, for example, a 5 minute duration. The trend to increasing variation at lower durations suggests that 5 minutes would have poorer reproducibility compared with $10 \mathrm{~min}$. In addition, a savings of 5 minutes per scan would only amount to 30-40 minutes of savings over the course of a 
day and would be unlikely to allow for additional animals to be imaged. A small study comparing a $5 \mathrm{~min}$ and $10 \mathrm{~min}$ acquisition would, however, be needed to confirm this hypothesis.

In our experiment we used a single rat species. Others (Vanhove C 2005) have suggested that different species may have different degrees of model variability. Therefore, though we believe that our results would be approximately correct for other rat species and other rat cardiac models, care should be taken in extending these results to those situations, particularly with respect to the inter-rat variability. Likewise, only male rats were used in this experiment and, while there is no expectation of gender differences, care should also be taken in extending these results to female rats.

We assumed in our experiment that the true value that was being measured does not change over the course of the experiment. For example, ejection fraction was assumed to be constant over time as long as the health of the animal was maintained. That is, we assumed that age itself does not change the value of ejection fraction. But, for the volume, one could argue that the uncertainty might change with age (weight) because the animals were growing with the time. Certainly the magnitude of the variation measured in $\mathrm{ml}$ is different for the MI rats (older) compared to the normals. This same argument applies to comparing EDV vs ESV. This is one of the reasons we also looked at the uncertainty as a percentage of the mean volume (Table 2.3). As a percent rather than an absolute value, the differences were less. So, we do not expect that the age will affect the reproducibility and the data we have comparing ESV and EDV uncertainty suggests that it did not. However, we did not try to compare reproducibility in younger animals with reproducibility in older animals. 
Although the normal and MI rats are of different ages we have used the same weight correction equation for both cases. Under normal circumstances, the weight of the rat is directly related to its age. However, the matter of concern is whether the relationship between weight and heart volume change as the weight of the rat increases. The data that we have in the normal rats show no such deviation from a linear relationship in the older (and hence heavier animals). A bigger problem with using the correction based on the normal rats is that these were healthy animals. It is possible that a disease state could alter the size of the heart independently of the weight of the rat. Let us consider a heart failure situation in which the disease state, as the disease progresses, leads to an enlarged heart volume. Thus, we know that at some point the relationship found for normal rats will not be applicable to infarcted animals (nor possibly to other disease models). Nevertheless, it serves as a means of performing at least a first order correction for this effect. In addition, as the duration of imaging for the MI-rat imaging is only a few weeks, the change in weight of the animals is reduced and the weight related change is correspondingly less, further reducing any error introduced by possible underlying inaccuracies in the application of the correction to infarcted rather than normal animals.

All of our images were reconstructed with a single set of reconstruction parameters. Our reconstruction parameters were based on the reconstruction of phantoms. Prior to the start of this study, a variety of different combinations were tried for the phantom and this choice of parameters seemed to give the best image quality for the count levels typical in a cardiac Tl-201 rat study. We could use several different reconstruction parameters which may have effect on the precision of our result. For example, if we would have used a heavier filter then the image would be 
more noise free and the uncertainty less but the image would be blurrier and it would be more difficult to determine the edges of the heart for volume estimation.

One more limitation of our experiment was the use of single operator. If more operators could have analyzed the data then we may get the variability of interobserver. Implications of one operator are that the variability is reduced over using multiple operators. Repeating the study with multiple operators would give an indication of how much worse this would be. For research studies, unlike clinical studies, it is easier to have everything evaluated by a single operator, so this might be a more representative uncertainty than it would be in a clinical setting.

\subsection{Conclusion}

With this study, for the first time, we have established the base-line inter- and intrasubject reproducibility of myocardial perfusion imaging on a microSPECT/CT with $\mathrm{Tl}-201$ in a rat model. 


\section{Chapter 3}

\section{Conclusion}

Micro-SPECT imaging of small animals has recently become a useful tool for cardiac research. The ability to perform serial imaging, monitoring the same animal over time, and to directly determine cardiac function, coupled with the non-invasive nature of the technology, make micro SPECT a valuable technique in the field of nuclear medicine research. Cardiac functional measurements like left ventricular volumes, perfusion and ejection fraction are important indices to assess cardiac diseases, determine functional status and predict outcomes. Gated SPECT is a reliable method to evaluate cardiac factors (Germano G 1995; Germano G 1998).

Rapidly growing knowledge in molecular science has suggested many new avenues for the development of new therapies and drugs. The sequencing of the human and the mouse genomes has led to a huge interest to pinpoint the genetic origin of diseases and disease progression. Small-animal molecular imaging is one of the main technologies being exploited to investigate these ideas. Small animal models accurately replicate human diseases and also the molecular process of disease progression. Imaging allows investigators to study these processes in vivo.

Single photon emission computed tomography has some important advantages over other methods of molecular imaging. A long history of the development of clinical tracers has led to a wide array of tracers for probing organ function. The availability of generators for the production of Tc99m and the long half-lives of other tracers like Tl-201 makes distribution of the tracers easier and has led to these tracers being widely commercially available at low costs. In addition, the sensitivity of 
nuclear medicine is excellent. With this modality, the tracer can be detected in nanoand pico- molar concentrations (Franc, BL 2008) allowing very small amounts of signal to be detected with little perturbation to the system. Over last 14 to 15 years, the usefulness of clinical SPECT has led to the design and construction of cameras dedicated for small animal use.

Micro SPECT has some special characteristics for small animal imaging. SPECT records $\gamma$-rays that are emitted directly from the radionuclide, avoiding the blurring caused by the positron range of positron emission tomography imaging and allowing for relatively high spatial resolution. Also SPECT can provide dual isotope imaging. It has the exclusive quality to image multiple probes with different isotopes which allows simultaneous study of multiple aspects of a disease. The radiopharmaceuticals used in SPECT are widely available. A final important advantage of microSPECT is that it generally costs less than small animal PET and MRI.

In this study we measured the reproducibility of cardiac functions in normal rats and in myocardial infarct (MI) rats. Pinhole gated myocardial perfusion micro SPECT/CT is appropriate for repetitive measurements of small animal cardiac function such as end diastolic volume, end systolic volume, ejection fraction and perfusion homogeneity. After scanning the rats, the OSEM iterative reconstruction algorithm was used to reconstruct the image from the gathered data. Images were analysed with 4DM-SPECT and the different cardiac functional parameters were calculated. The repeated measurements in our study allowed us to establish mean values for healthy and infarcted animals, and for the first time, to establish values for the intra- and inter-rat reproducibility of these values. For example, we have the EDV of $452 \pm 4 \mathrm{~mm}^{3}, \mathrm{ESV}$ of $208 \pm 2 \mathrm{~mm}^{3}$ and EF of $54 \% \pm 0.3 \%$ for 8 normal rats and 
for $8 \mathrm{MI}$ rats the EDV, ESV and EF were $664 \pm 3 \mathrm{~mm}^{3}, 325 \pm 4 \mathrm{~mm}^{3}$ and $51 \% \pm 1 \%$ respectively. These values are the mean and uncertainty obtained for a 30 minute scan duration.

Our study with Tl-201 was in a good agreement with Tc-99m (Table 2.4) which indicates that Tl-201 can be a substitute for Tc-99m during the recent shortage of Tc-99m. One of the unique characteristic of SPECT is multi-isotope imaging which is very important while assessing the heart. SPECT is able to distinguish the signals from different radiotracers using energy discrimination and can image multiple signals simultaneously. With this exclusive characteristic of SPECT, perfusion imaging of Tl-201 allows the use of Tc-99m labelled radiotracer to obtain other features of the object. Thus not only a substitute of Tc-99m, Tl-201 will be a good isotope for dual isotope imaging in future. Multiple isotope imaging increases the data from a single animal and decreases the duration of imaging. Other isotope combinations are also possible. We also can use In-111 or I-123 for simultaneous imaging of multiple signals with Tl-201. However, while good in theory, interference from the emissions of the other isotopes will potentially degrade image quality and increase the uncertainty of image-derived measurements. Thus, further studies will be needed to determine the impact of multi-isotope imaging on Tl-201 cardiac measurements.

Other studies have also investigated the left ventricular functions in rats. Though there is variability amongst published values, our data are consistent with that seen by others with other modalities. Vanhove et al (Vanhove C 2005) reported the inter-study variability of EDV is $704.0 \pm 30 \mu 1$, ESV is $182 \pm 8 \mu 1$ and EF is $74 \% \pm 1$ $\%$ for normal rats using pinhole gated SPECT. Wise et al showed the EDV of $463 \pm$ $36 \mu 1$ and EF of $58 \% \pm 1 \%$ using 8 normal rats with MRI technique (Wise RG 1998; 
Wise RG 1999). Nahrendorf et al also reported the result of cardiac functions using MRI for both normal and MI rats. They ended with the EDV of $737.0 \pm 70.5 \mu 1, \mathrm{ESV}$ of $480.7 \pm 63.7 \mu \mathrm{l}$ and $\mathrm{EF}$ of $36.4 \pm 2.5 \%$ for $11 \mathrm{MI}$ rats and for 4 normal rats the EDV was $316.1 \pm 16.3 \mu 1, \mathrm{ESV}$ was $91.7 \pm 4.7 \mu \mathrm{l}$ and $\mathrm{EF}$ was $70.6 \pm 2.0 \%$. Croteau et al (Croteau E 2003) have compared the same cardiac factors using PET. They demonstrated that the EDV was $496 \pm 41 \mu 1$ and the average EF was $81.6 \% \pm 1.8 \%$. They used 11 normal Sprague-Dawley rats in their experiment.

Future work on this topic could address some of the limitations inherent in this study design. One of the limitations of our experiment was that all of the analysis was done by a single operator. If there were different operators analyzing all of the images, then variation due to operator could be obtained and the experiment would be more generalizable. So if we analyzed the data with multiple operators then it would show the influence of multiple operator on the reproducibility which may or may not be different than current study. Vanhove et al (Vanhove C 2005) found no significant differences in inter-observer reproducibility.

Additionally, the reconstruction algorithm that we used in our study did not include attenuation correction. The reproducibility calculation would be affected by the attenuation correction. Reanalyzing this data with the attenuation correction would allow us to understand the influence of this factor. While attenuation is expected to be low due to the small size of the subjects, because of the low energy of the primary emission of Tl-201 $(\sim 70 \mathrm{keV})$, the impact of attenuation will still be greater than with the more commonly used Tc-99m-tracers.

Our study was only with a single species, the rat. Thus, we cannot comment on the reproducibility of measurements of other species. Further studies of a similar 
design are still needed to establish the uncertainty in other animals, particularly in the mouse. Such a study would also allow comparison of the reproducibility for different species.

The current study provides the baseline reproducibility of Tl-201 for cardiac microSPECT imaging. In future this baseline reproducibility can be used for the assessment of reconstruction algorithm improvement. Myocardial perfusion SPECT has also the benefit to provide information of left ventricular functions, wall motion and perfusion. Baseline reproducibility allows for sample size calculations in studies of cardiac disease, like interventions such as beta blockers on progression of disease following MI. The better understanding of uncertainty provided by this study will allow more effective design of studies such as these and move forward the realization of microSPECT imaging as a valuable tool for cardiac research. 


\section{References}

Aitman TJ, Critser JK, Cuppen E et. al. (2008). "Progress and prospects in rat genetics: a community view. " Nat Genet 40: 516-522.

Beck RN, Zimmer LT, Charleston DB, et al (1973). "Advances in fundamental aspects of imaging systems and techniques, in Medical Radioisotope Scanning." Vienna:IAEA 1: 3-45.

Berthelot JM, Pulcins I,Wen E, et al. (2010). "Health Indicators 2010". Statistics Canada, Ottawa, Canada

Bok BD, Bice AN, Clausen M, et al (1987). "Artifacts in camera based single photon emission tomography due to the time activity variation." Eur J Nucl Med 13: 439-442.

Bonow RO, Ritchie JL, Garcia EV, et al. (1992). "Standardization of cardiac tomographic imaging." J Nucl Med 33:1434-1435.

Boucher CA, Zir LM, Beller GA, et al. (1980). "Increased lung uptake of thallium201 during exercise myocardial imaging: clinical, hemodynamic and angiographic implications in patients with coronary artery disease." Am J Cardiol 46: 189-196. 
Bruyne SDe (2009). "Design and evaluation of novel PET and SPECT tracers for imaging the monoamine system and the $\mathrm{P}-\mathrm{gp}$ transporter." $\underline{\mathrm{PhD}}$. Thesis.

Cerqueira MD, Weissman NJ, Dilsizian V, et al. American Heart Association Writing Group on Myocardial Segmentation and Registration for Cardiac Imaging. (2002). "Standardized myocardial segmentation and nomenclature for tomographic imaging of the heart: a statement for healthcare professionals from the Cardiac Imaging Committee of the Council on Clinical Cardiology of the American Heart Association." Circulation 105: 539-542.

Cherry SR, Sorenson JA and Phelps ME. (1987). "Physics in Nuclear Medicine." Orlando: Grune \& Stratton.

Cherry SR, Sorenson JA and Phelps ME (2003) "Physics in Nuclear Medicine." Elsevier, Philadelphia, USA.

Cherry SR. (2004). "In vivo molecular and genomic imaging: new challenges for imaging physics." Phys Med Biol. 49: R13-R48.

Cho ZH, Jones Joie P and Singh M (1993). "Foundations of Medical Imaging." John Wiley \& Sons, New York, USA. 
Choy JB and Leslie WD. (2001). "Clinical correlates of Tc-99m sestamibi lung uptake." J Nucl Cardiol 8(6): 639-644.

Clark AN and Beller GA (2005). "The present role of nuclear cardiology in clinical practice." Q J Nucl Med Imaging 49(1): 43-58.

Constantinesco A, Choquet P, Monassier L, Israel-Jost V and Mertz L. (2005). "Assessment of Left Ventricular Perfusion, Volumes, and Motion in Mice Using Pinhole Gated SPECT." J Nucl Med 46(6): 1005-1011.

Croteau E, Benard F, Cadorette J, Gauthier ME, Aliagra A, Bentourkia M, Lecomte R. (2003). "Quantitative gated PET for the assessment of left ventricular function in small animals." J Nucl Med 44: 1655-1661.

DePuey EG, Garcia EV. (1989). "Optimal specificity of thallium-201 SPECT through recognition of imaging artifacts." J Nucl Med 30(4):441-9

Depuey EG, Garcia EV, Berman DS (2001). "Cardiac SPECT Imaging." Lippincott Williams \& Wilkins, Philadelphia, USA.

Depuey EG. (2006). "IMAGING GUIDELINES FOR NUCLEAR CARDIOLOGY PROCEDURES: A Report of The American Society of Nuclear Cardiology Quality Assurance Committee." American Society of Nuclear Cardiology. 
Di Filippo FP. (2008). "Design and performance of a multi-pinhole collimation device for small animal imaging with clinical SPECT and SPECT-CT scanners." Phys Med Biol 53: 4185-4201.

Doggrell SA, Brown L (1998). "Rat models of hypertension, cardiac hypertrophy and failure." Cardiovascular Research 39: 89-105.

Everaert H, Farnken PR, Flamen P, Goris M, Momen A and Bossuyt A (1996). "Left ventricular ejection fraction from gated SPET myocardial perfusion studies: a method based on the radial distribution of count rate density across the myocardial wall." Eur J Nucl Med 23: 1628-1633.

Fishbein MC, Maclean D, Maroko PR (1978). "Experimental myocardial infarction in the rat: qualitative and quantitative changes during pathologic evolution." Am J Pathol 90(1): 57-70.

Forrer F, Valkem R, Bernard B, Schramm NU, Hoppin JW, Rolleman E, Krenning EP, Jong M de (2006). "In vivo radionuclide uptake quantification using a multipinhole SPECT system to predict renal function in small animals." Eur J Nucl Med Mol Imaging 33: 1214-1217. 
Franc BL, Acton PD, Mari C, and Hasegawa BH (2008). "Small-Animal SPECT and SPECT/CT: Important Tools for Preclinical Investigation." J Nucl Med. 49: 16511663.

Germano G, Kiat H, Kavanagh PB, Moriel M, Mazzanti M, Su HT, Van Train KF and Berman DS. (1995). "Automatic quantification of ejection fraction from gated myocardial perfusion SPECT." J Nucl Med. 36(11): 2138-2147.

Germano G, Kavanagh PB, Kavanagh JT, Wishner SH, Berman DS, Kavanagh GJ. (1998). "Repeatability of automatic left ventricular cavity volume measurements from myocardial perfusion SPECT." J Nucl Cardiol. 5(5): 477-483.

http://www.physics.ubc.ca/ mirg/home/tutorial/fbp recon.html

Hudson HM, Larkin RS. (1994). "Accelerated image reconstruction using ordered subsets of projection data." IEEE Trans Med Imaging 13(4): 601-609.

Ishizu K, Mukai T, Yonekura Y, et. al. (1995). "Ultra-high Resolution SPECT System Using Four Pinhole Collimators for Small Animal Studies." J Nucl Med 36(22822287). 
Iskander S, and Iskandrian AE (1998). "Risk Assessment Using Single-Photon Emission Computed Tomographic Technetium-99m Sestamibi Imaging." J Am Coll Cardiol 32(1): 57-62.

Iskandrian AE, Germano G, Vandecker W, Ogliby JD, Wolf N, Mintz R, et al. (1998). "Validation of left ventricular volume measurements by gated SPECT $99 \mathrm{mTc}-$ labeled sestamibi imaging." J Nucl Cardiol. 5: 574-578.

Jacob HJ (1999). "Functional genomics and rat models." Genome Res. 9: 1013-1016

Jansen FP, Vanderheyden JL. (2007). "The future of SPECT in a time of PET." Nucl Med Biol 34(733-735).

Jaszczak RJ, Coleman RE, Lim CB (1980). "SPECT: Single photon emission computed tomography." IEEE Trans Nucl Sci NS 27: 1137-1153.

Jaszczak RJ, Coleman RE and Whitehead FR (1981). "Physical factors affecting quantitative measurements using camerabased single-photon emission computed tomography (SPECT)." IEEE Trans Nucl Sci NS 28: 69-80.

Jaszczak RJ and Coleman RE. (1985). "Single photon emission computed tomography (SPECT) principles and instrumentation." Invest Radiol 20: 897-910. 
Johns HE and Cunningham JR (1983). "The Physics of Radiology." Charles C Thomas - Publisher, Illinois, USA.

King MA, Pretorius PH, Farncombe T and Beekman FJ. (2002). "Introduction to the Physics of Molecular Imaging With Radioactive Tracers in Small Animals." J. Cell. Biochem. Suppl. 39: 221-230.

Knoll GF. (1979). "Radiation detection and measurement." Wiley: chap 10, New York, USA

Lalush DS and Tsui BMW (2000). "Performance of ordered-subset reconstruction algorithms under conditions of extreme attenuation and truncation in myocardial SPECT." J. Nucl. Med. 41: 737-744.

Levin CS. (2005 Dec). "Primer on molecular imaging technology." Eur J Nucl Med Mol Imaging. 32(Suppl 2): S325-345.

Links JM TL Frank, Becker LC (1991). "Effects of different tracer washout during SPECT acquisition." J Nucl Med. 32: 2253-2257.

Mackay J and Mensah G. (2004) "The Atlas of Heart Disease and Stroke." World Health Organization, Geneva, Switzerland. 
Mahmood U and Weissleder R. (2001). "Molecular Imaging." Radiology 291(316$333)$.

Manglos SH, Jaszczak RJ, Floyd CE, et al (1987). "Nonisotropic attenuation in SPECT: Phantom test of quantitative effects and compensation techniques." $\mathrm{J}$ Nucl Med. 28: 1584-1591.

Meikle SR, Kench P, Kassiou M and Banati RB. (2005 ). "Small animal SPECT and its place in the matrix of molecular imaging technologies." Phys Med Biol. 50(22): R45-61.

Metz CE, Atkins FB, Beck RN (1980). "The geometric transfer function component for scintillation camera collimators with straight parallel holes." Phys Med Biol. 25: 1059-1070.

Peremans K, Bart, Van Den BB, Kurt A and Van de WC (2005). "A review of small animal imaging planar and pinhole SPECT $\gamma$ camera imaging." Vet Radiol Ultrasound 46: $162-170$.

Pourmoghadas A. (2010). "Respiratory Motion-Correction in 82-Rb Cardiac PET/CT." M.Sc Thesis Department of Physics, Carleton University. 
Rowland DJ and Cherry SR (2008 Elsevier Inc). "Small- Animal Preclinical Nuclear Medicine Instrumentation and Methodology." Semin Nucl Med 38: 209-222.

Saha GB. (2003). "Fundamentals of Nuclear Pharmacy." Springer Fifth Edition, Verlag, NY, USA.

Sciagra R and Leoncini M (2005). "Gated single-photon emission computed tomography. The present-day "one-stop-shop" for cardiac imaging." $\underline{Q}$ J Nucl Med 49(1): 19-29.

Sciagra R. (2007). "The expanding role of left ventricular functional assessment using gated myocardial perfusion SPECT: the supporting actor is stealing teh scene." Eur J Nucl Med Mol Imaging 34: 1107-1122.

Sharir T, Germano G, Kang X, Lewin HC, Miranda R, Cohen I, et al. (2001). "Prediction of myocardial infarction versus cardiac death by gated myocardial perfusion SPECT: risk stratification by the amount of stress-induced ischemia and the post-stress ejection fraction." J Nucl Med. 42: 831-837.

Sharir T. (2007). "Gated myocardial perfusion imaging for the assessment of left ventricular function and volume: From SPECT to PET." J Nucl Cardiol. 14: 631-633. 
Statistics Canada (2010), "Leading Causes of Death in Canada, 2007”, Catalogue no. 84-215-x2010001.

Strydhorst J, Lockwood J, Kordos M, Leenen F, Ruddy TD, Wells RG. (2010). "Reproducibility of Tc99m-tetrofosmin cardiac gated microSPECT measurements in rats." J Nucl Med 51(Suppl. 2): 475.

Tsui BMW. (1988). "Collimator design, properties and characteristics, in Simmons GH (ed): The Scintillation Camera." The Society of Nuclear Medicine chap 2: 17-45, NY, USA.

Tsui BMW (1992). "Reconstruction and filtering methods for quantitative cardiac SPECT imaging, in Reiber JHC, van der Wall EE (eds): Cardiovascular Nuclear Medicine and MRI: Quantitation and Clinical Applications." Kluwer Academic,: 2946, Dordrecht, The Netherlands.

Tsui BMW, Zhao X, Frey EC, McCartney WH (1994). "Quantitative Single Photon Emission Tomography: Basics andClinical Considerations." Semi Nucl Med 24(1): $38-65$.

Tsui BMW (1996). "Physics of SPECT." Radiographics 16: 173-183.

Vanhove C, Defrise M, Franken PR, Everaert H, Deconinck F, Bossuyt A. (2000). "Interest of the ordered subsets expectation maximization (OS-EM) algorithm in 
pinhole single-photon emission tomography reconstruction: a phantom study." Eur J Nucl Med 27(2): 140-146.

Vanhove C, Walgraeve N, Geeter F De and Franken PR. (2002). "Gated myocardial perfusion tomography versus gated blood pool tomography for the calculation of left ventricular volumes and ejection fraction." Eur J Nucl Med 29(6):735-741.

Vanhove C, Lahoutte T, Defrise M, Bossuyt A, Franken PR. (2005). "Reproducibility of left ventricular volume and ejection fraction measurements in rat using pinhole gated SPECT." Eur J Nucl Med Mol Imaging 32(2): 211-220.

Wackers FJ. (1994). "Exercise Myocardial Perfusion Imaging." J Nucl Med 35: 726.

Webb S. (1988). "The Physics of Medical Imaging." Institute of Physics Publishing, Bristol, CT.

Weber DA and Ivanovic M (1999). "Ultra-high-resolution imaging of small animals: Implications for preclinical and research studies." J Nucl Cardiol 6: 332-344.

Wernick MN and Aarsvold JN (2004). "Emission Tomography: The fundamentals of PET and SPECT." Elsevier academic press. Amsterdam, The Netherlands. 
Wielgosz A, Arango M, Bancej C, et al. (2009). "Tracking Heart Disease and Stroke in Canada". Public Health Agency of Canada, Ottawa, Canada

Wise RG, Huang CL, Gresham GA, Al-Shafei Al, Carpenter TA, Hall LD. (1998). "Magnetic resonance imaging analysis of left ventricular function in normal and spontaneously hypertensive rats." J Physiol 3: 873-887.

Wise RG, Huang CL, Al-Shafei Al, Carpenter TA, Hall LD. (1999). "Geometric models of left ventricular contraction from MRI of the normal and spontaneously hypertensive rat heart." Phys Med Biol. 44: 2657-2676.

Yalcin H, Maza S and Yalcin F (2008). "Single photon emission computed tomography: An alternative imaging modality in left ventricular imaging." Vasc Health Risk Manag 4(5): 1069-1072. 


\section{Appendices}

(A) Permission of the figure 1.5: Different reconstructed images

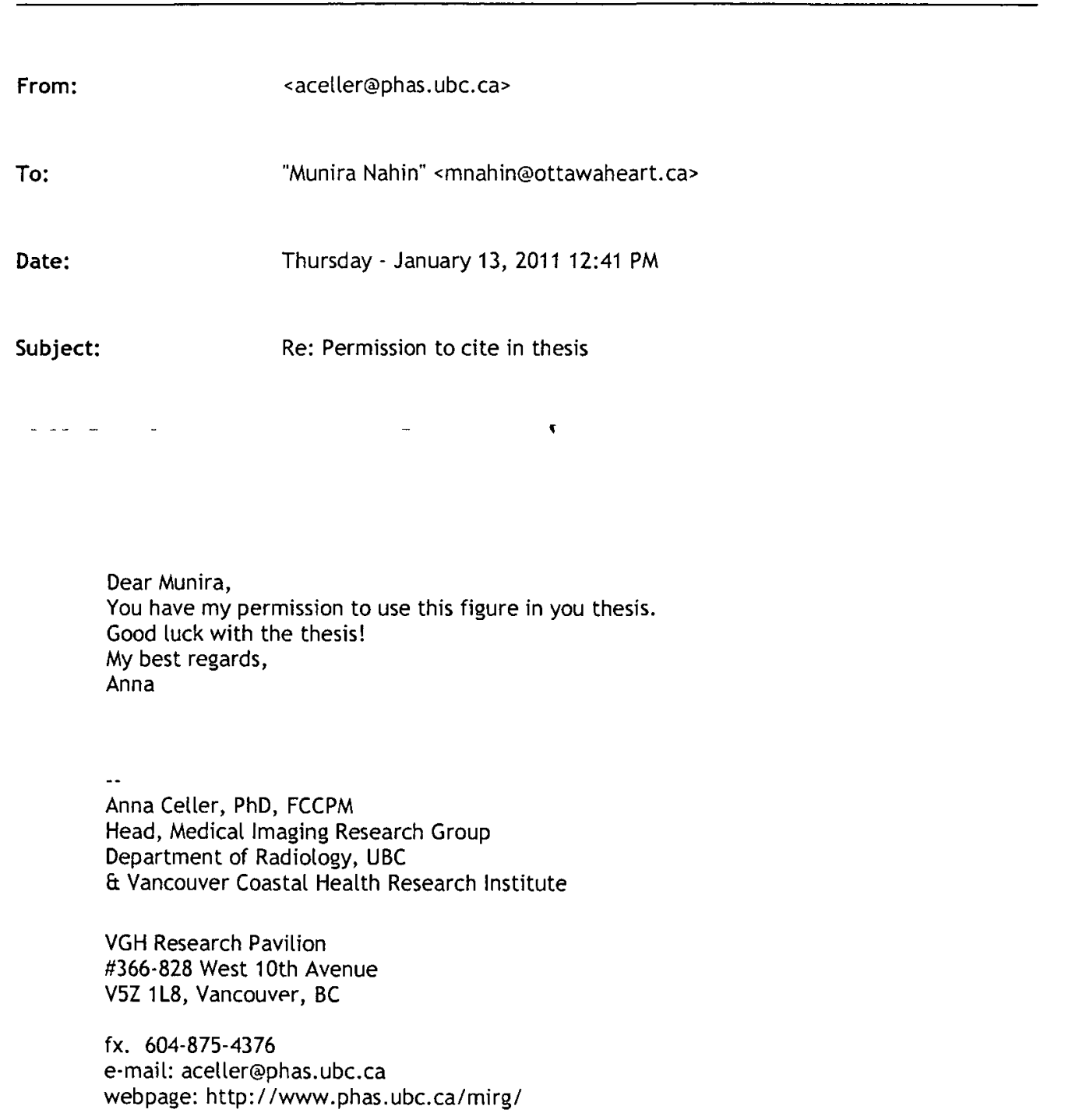


(B) Permission of the figure 1.7: Polar map representation with the corresponding 3D LV image

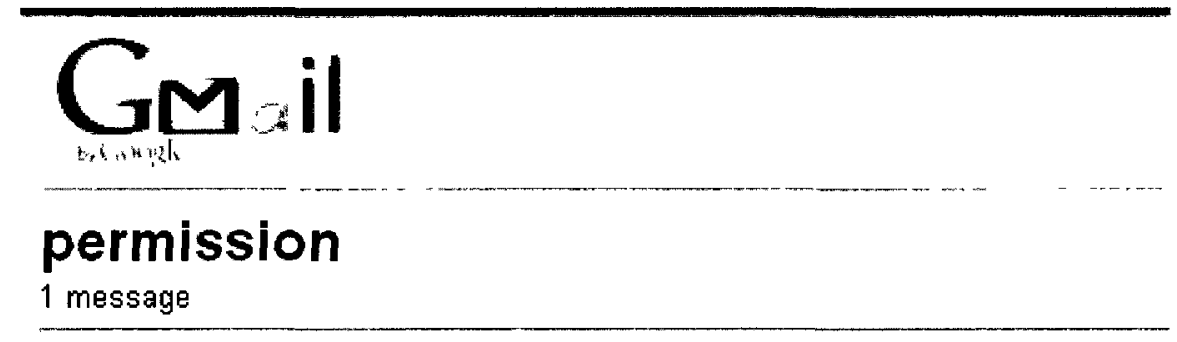

Amir Pourmoghadas < amirpourmoghadasayahoo.com>

Reply-To: Amir Pourmoghadas <amirpourmoghadas@yahoo.com>

To: munirafardous <munira.fardous@gmail.com>

Hi Munira,

You have my permission to use this figure in your thesis.

Good luck!

Amir

Amir Pourmoghaddas

$\mathrm{PhD}$ Physics Candidate

Physics Dept., Carleton University

Cardiac PET Imaging Dept.

University of Ottawa Heart Institute

40 Ruskin st, Ottawa, Ontario, K1Y 4W7

H-1204 PET Research 1

(613) 798-5555 ext. 13586

email: apour@ottawaheart.ca 
(C) Sample calculations of weight corrected EDV for normal rats:

\begin{tabular}{|c|c|c|c|c|c|c|c|c|}
\hline EDV & Week 1 & Week 2 & Week 3 & Week 4 & Week 5 & & & \\
\hline & Scan 1 & Scan 2 & Scan 3 & Scan 4 & Scan 5 & SD & $\begin{array}{l}\text { Average } \\
\text { SD }\end{array}$ & Std Error \\
\hline Rat 1 & 405.8681737 & 477.1676383 & 442.192695 & \begin{tabular}{|l|}
457.7899227 \\
\end{tabular} & 416.720238 & \begin{tabular}{|l|}
29.19605 \\
\end{tabular} & & \\
\hline Rat 2 & \begin{tabular}{|l}
395.1819386 \\
\end{tabular} & 449.7993418 & 450.485235 & 427.6208071 & 401.1841533 & 26.11311 & 29.82948 & 3.195254 \\
\hline Rat 3 & 462.2948048 & 454.2450367 & 469.618374 & 480.2119284 & 500.5985794 & 17.96318 & & \\
\hline Rat 4 & 406.9386123 & 422.4570991 & 495.304859 & 507.313156 & 434.7669372 & 45.07203 & & \\
\hline Rat 5 & 408.1956193 & 442.6147644 & 456.527613 & 449.6835694 & 454.2308768 & 19.76413 & & \\
\hline Rat 6 & 469.9505461 & 487.8718682 & 498.631765 & 480.4155224 & 421.9078607 & 29.77042 & & \\
\hline Rat 7 & 518.5037437 & 534.7503292 & 545.933321 & 590.0633732 & 587.6942648 & 32.10074 & & \\
\hline Rat 8 & 401.4700682 & 500.0675806 & 485.98117 & 478.3813517 & 455.2480168 & 38.65615 & & \\
\hline SD & 44.70047359 & 36.10291131 & 33.638565 & 49.12793017 & 60.26463148 & & & \\
\hline $\begin{array}{l}\text { Average } \\
\text { SD } \\
\end{array}$ & & & 44.7669023 & & & & & \\
\hline $\begin{array}{l}\text { Std } \\
\text { Error }\end{array}$ & & & 4.785954544 & & & & & \\
\hline
\end{tabular}

Above is a data table for the measurements of EDV for normal rats and a scan duration of $10 \mathrm{~min}$.

Standard Deviation (Across Scans) calculated using the formula:

$$
S D=\sqrt{\frac{\sum_{1}^{n}\left(x_{i}-\bar{x}\right)^{2}}{(n-1)}}
$$

Here $\mathrm{x}_{\mathrm{i}}=$ each score, $\bar{x}=$ mean, $\mathrm{n}=$ number of observations. Applying this to

Rat 1,

$S D$

$=\sqrt{\frac{(406-440)^{2}+(477-440)^{2}+(442-440)^{2}+(458-440)^{2}+(417-440)^{2}}{5-1}}$

$=29$ 
Average SD (Across Scans) calculated using the formula:

$$
\text { Average } S D=\frac{\sum_{1}^{n} x_{i}}{n}
$$

Applying this to Rat 1,

$$
\text { Average SD }=\frac{29+26+18+45+20+30+32+39}{8}=30
$$

Standard Error (Across Scan) calculated using the formula:

$$
\text { Std. Error }=\frac{S D}{\sqrt{n}}
$$

Applying this to Rat 1, we found,

Std Error $=$

$\frac{(29-30)^{2}+(26-30)^{2}+(18-30)^{2}+(45-30)^{2}+(20-30)^{2}+(30-30)^{2}+(32-30)^{2}+(39-30)^{2}}{\sqrt{8}}=$

3 
